\title{
Worldwide cross-ecosystem carbon subsidies and their contribution to ecosystem functioning
}

\author{
Isabelle Gounand $^{*, 1,2}$, Chelsea J. Little ${ }^{1,2}$, Eric Harvey ${ }^{1,2,3}$, and Florian Altermatt ${ }^{1,2}$
}

Corresponding author: Isabelle Gounand - isabelle.gounand@eawag.ch

Affiliations:

${ }^{1}$ University of Zurich, Department of Evolutionary Biology and Environmental Studies, Winterthurerstr. 190, CH-8057 Zürich, Switzerland.

${ }^{2}$ Eawag: Swiss Federal Institute of Aquatic Science and Technology, Department of Aquatic Ecology, Überlandstrasse 133, CH-8600 Dübendorf, Switzerland

${ }^{3}$ University of Toronto, Department of Ecology and Evolutionary Biology, Toronto, Canada, M5S 3B2

E-mail addresses:

IG: isabelle.gounand@eawag.ch

CJL: chelsea.jean.little@gmail.com

EH: eric.harvey@utoronto.ca

FA: florian.altermatt@eawag.ch 


\begin{abstract}
Ecosystems are widely inter-connected by spatial flows of resources ${ }^{1,2}$, yet primarily studied in a local context. Meta-ecosystem models suggest that cross-ecosystem subsidies can play an essential role in ecosystem functioning, notably by controlling local availability of resources for biological communities ${ }^{3-6}$. The general contribution of these resource connections to ecosystem functioning, however, remains unclear in natural systems, due to the heterogeneity and dispersion of data across the ecological literature. Here we provide the first quantitative synthesis on spatial flows of carbon connecting ecosystems worldwide. These cross-ecosystem subsidies range over eight orders of magnitude, between $10^{-3}$ and $10^{5} \mathrm{gC} \mathrm{m}^{-2} \mathrm{yr}^{-1}$, and are highly diverse in their provenance. We found that spatial carbon flows and local carbon fluxes are of the same order of magnitudes in freshwater and benthic ecosystems, suggesting an underlying dependency of these systems on resources provided by connected terrestrial and pelagic ecosystems respectively. By contrast, in terrestrial systems, cross-ecosystem subsidies were two to three orders of magnitude lower than local production (grasslands and forests), indicating a weaker quantitative influence on functioning. Those subsidies may still be qualitatively important, however, as some have high nutrient content ${ }^{7,8}$. We also find important gaps in carbon flow quantification, notably of cross-ecosystem subsidies driven by animal movements, which likely leads to general underestimations of the magnitude and direction of cross-ecosystem linkages ${ }^{9}$. Overall, we demonstrate strong ecosystem couplings, suggesting that ecosystems can be vulnerable to alterations of these flows and pointing to an urgent need to re-think ecosystem functioning in a spatial perspective.
\end{abstract}




\section{Main text}

Ecosystems and the services that they provide are essential for material and cultural human welfare ${ }^{10,11}$, but paradoxically, human activities threaten ecosystem integrity $^{12,13}$. Maintaining functional ecosystems, or restoring degraded ones, requires the identification of dominant mechanisms driving their dynamics. At the local scale, ecologists have accumulated extensive data on individual ecosystems' functioning ${ }^{14}$. Accurate depictions of within-ecosystem fluxes, such as biomass and detritus production, respiration, or decomposition, exist for all broad ecosystems types, including terrestrial, freshwater and marine ones.

But ecosystems are not isolated. For flows of dispersing organisms the role on large-scale species coexistence and community dynamics is well studied ${ }^{15,16}$. However, it remains unclear to which extent ecosystem functioning also depends on cross-ecosystem flows of resources, such as in the form of detritus or nutrients ${ }^{1,17}$. According to the recently developed meta-ecosystem theory ${ }^{16,18}$, such cross-ecosystem resource flows can induce strong interdependencies between ecosystems and drive ecosystem functioning ${ }^{3-6}$. While the literature on subsidies provides emblematic cases of resources moving between ecosystems, including passive transport of leaves windblown from forests to streams ${ }^{2}$, or active transport such as aquatic insects emerging onto land ${ }^{19}$, we still lack a general quantification of these flows. The dispersion of data over many research areas and inconsistencies in the units of measurements used has hitherto precluded a general and synthetic overview of resource spatial flows.

Here, we conduct the first quantitative synthetic assessment of cross-ecosystem subsidies connecting the major ecosystem types across the globe (Figure 1). Specifically, we compare the magnitudes of cross-ecosystem subsidies to within-ecosystem fluxes in order to infer their relative contribution to ecosystem functioning. This also gives a basis to identify ecosystems' vulnerability to increasing alterations of resource flows under the context of ongoing global changes ${ }^{20}$. We based our analysis on generally convertible estimates in units of carbon ( $\mathrm{gC} \mathrm{m}^{2} \mathrm{y}^{-1}$; see Methods) and systematically searched for 
quantifications of spatial subsidies connecting terrestrial (forest, grassland, agroecosystem, desert), freshwater (stream, lake), and marine (pelagic and benthic) ecosystems. To compare these spatial flows, we also assembled comprehensive quantifications of local biological fluxes of carbon (i.e., gross primary production, ecosystem respiration, and decomposition; see Methods) within the different ecosystem types. We assembled 518 measurements of spatial flows and 2516 of local fluxes, reaching a total of 3034 data points extracted from 557 studies. Analysing this data set with its internally-consistent measurements in carbon units reveals for the first time the widespread importance of spatial resource flows to local ecosystem functioning.

Spatial flows of cross-ecosystem carbon subsidies range over eight orders of magnitude of mass of carbon annually transported to ecosystems $\left(\mathrm{gC} \mathrm{m}^{-2} \mathrm{y}^{-1}\right)$, from a few milligrams (aquatic insects deposited in forest) to more than ten tons per meter squared and year (wrack on shores) (Figure 2). The materials transported among ecosystems are as various as living animals, dead plants and animals, and dissolved carbon. Ecosystems occupying the lowest elevations in land- and seascapes receive downward flows of dead material or small organisms falling from above ecosystems, while terrestrial ecosystems receive more lateral flows, for instance of material transported by wind, ocean tides, or animal movements. Not surprisingly, ecosystems dominated by primary producer biomass (e.g., forests, grasslands, submarine meadows, and kelp forests), export the largest flows of primary production-derived material (median / interquartile range [IQR] of 148.7 / [47.1-246.6] and 164 / [93.2-1659.3] $\mathrm{gC} \mathrm{m}^{-2} \mathrm{yr}^{-1}$ for terrestrial vegetation and macro-algae, respectively). By comparison, invertebrate subsidies are substantially lower (median: $1.51 \mathrm{gC} \mathrm{m}^{2} \mathrm{y}^{-1} / \mathrm{IQR}$ : [0.44-5.41]), but might reach similar values in specific ecosystems (e.g., $124 \mathrm{gC} \mathrm{m}^{2} \mathrm{y}^{-1}$ of aquatic insects flowing from lakes to tundras in Iceland). Lastly, few studies document spatial flows of vertebrate origin (3.1\%), and those which do report highly contrasting values: faeces of foraging deer or fishery discards represent small flows of around $0.1-1 \mathrm{gC} \mathrm{m}^{2} \mathrm{y}^{-1}$, while the net inputs to freshwater ecosystems of hippopotamus defecating into rivers, or of drowned migrating wildebeests, range between 100 and $1000 \mathrm{gC} \mathrm{y}^{-1}$ per meter squared of river. These disparities stress that the quantitative effect of carbon brought to ecosystems via large 
animals might greatly depend on how resource deposition is spatially constricted to small or large areas. Overall, our global picture highlights the ubiquity of cross-ecosystem subsidies, and the variety in their magnitude.

To assess the importance of these subsidies to ecosystem functioning, we compared their magnitude to those of local biological fluxes within each receiving ecosystem. Magnitudes of subsidies versus local fluxes are similar in freshwater and in some benthic systems, whereas subsidies are generally far smaller than local fluxes in terrestrial ecosystems (Figure 3). This results both from more abundant cross-ecosystem subsidies in the direction of freshwater and benthic ecosystems than terrestrial and pelagic systems, and from substantially lower primary production in the former. Ecosystem respiration in freshwater and benthic systems often exceeds local primary production, despite a noticeable variability, for example due to differences in light availability in shallow tropical sea grass meadows versus deep waters promoting or constraining photosynthesis respectively. Ecosystem heterotrophy (see negative net ecosystem production in Extended data Figure 1) is associated with cross-ecosystem inflows of comparable or greater magnitude than local production, indicating that functioning in these ecosystems depends substantially on allochthonous resources. This dependency makes freshwater and deep unproductive benthic systems sensitive to alterations of these resources and, thus, to donor ecosystem dynamics ${ }^{21,22}$.

By contrast, terrestrial and pelagic ecosystems tend to have a net autotrophic functioning and receive negligible subsidies compared to their local production, except in deserts (Figure 3). In the latter case, where local production is limited (e.g., by water availability), the potential impact of subsidies provided by other, less limited ecosystems (e.g., oceans) increases. Otherwise, autotrophic ecosystems seem relatively free from influence of spatial resources (or at least, from the influence of aquatic subsidies: we found only two studies reporting subsidies between terrestrial ecosystems). However, carbon subsidies are intimately linked to nutrients within biological molecules. Though quantitatively small in terms of carbon, subsidies to terrestrial systems are of higher quality with respect to nitrogen content than the vegetation-based subsidies they export 
(e.g. insects versus leaves in terrestrial-freshwater couplings ${ }^{8,23}$ ). Some cross-ecosystem subsidies, not considered here, are even predominantly of nitrogen or phosphorus, enriching terrestrial (e.g., guano from foraging sea birds ${ }^{24}$ ) or pelagic systems (e.g., excretion of marine mammals ${ }^{25}$ ). Carbon and nutrients in cross-ecosystem subsidies may relax different limitations in recipient ecosystems, thus enhancing regional production ${ }^{6,26}$.

Overall, our synthesis of carbon-based cross-ecosystem subsidies identifies strong spatial couplings between autotrophic and heterotrophic ecosystems (Figure 4).

Cataloguing the types of subsidies documented so far, and their drivers (Figure 1), also reveals important gaps in the assessment of ecosystem couplings. A large portion of the quantified subsidies concerns flows occurring at terrestrial-freshwater (66.9\%) or pelagicbenthic (18.0\%) interfaces, and are driven by passive rather than active processes (Figure $2 c ; 70.5 \%$ ). This may be because passive flows of detritus or of small organisms can be easily measured by various traps. By contrast, it is more challenging to quantify active spatial flows resulting from animal movement. This requires indirect estimations, for instance combining animal tracking and measurements of ingestion and excretion rates ${ }^{27}$. Quantifications are rare (Figure 2), but the few existing studies suggest that widespread animal movements, such as foraging or migration, could act as important ecosystem connectors $^{28}$. In particular, mobile species using multiple ecosystems ${ }^{29}$, or those whose behaviour leads to massive aggregations of individuals, likely move important amounts of resources among ecosystems. At the moment, the lack of data hinders an assessment of the global importance of these actively-driven cross-ecosystem subsidies ${ }^{9}$. However, such linkages could render common ecosystem couplings more bi-directional than previously thought, as proposed for the aquatic-terrestrial interface ${ }^{8,30}$. Detailed evaluation of flow bi-directionality is essential to better understand spatial feedbacks between connected ecosystems. Resource exchanges between ecosystems underlie both optimization in resource use at the landscape scale, and potentials for spatial amplification of local perturbations.

In summary, the magnitude and importance of cross-ecosystem carbon subsidies for ecosystem functioning is globally variable, but predictable based on general 
knowledge of internal ecosystem fluxes and metabolism (net heterotroph vs. autotroph). Freshwater and benthic ecosystems might be especially vulnerable to subsidy alteration, and to perturbations in autotrophic ecosystems spatially cascading via these subsidies. However, gaps in subsidy quantification leave some uncertainty as to whether different ecosystems might act as buffers or amplifiers of spatial dynamics within landscapes.

Documenting these ecological blind spots is necessary to improve our ability to predict ecosystem responses to global changes across landscapes. 


\section{Methods}

We conducted an extensive literature review of empirical values of cross-ecosystem subsidies over the globe (distribution in Figure 1), and compared their magnitude to local fluxes within ecosystems receiving these subsidies. We chose carbon as the focal material unit to profit from widely available carbon data for local fluxes. To enable the spatial flows to local flux comparison, we only considered measurements of spatial flows that were either provided in or could be converted into $\mathrm{gC} \mathrm{m}^{-2} \mathrm{yr}^{-1}$.

\section{Data collection}

Our systematic search covered four broad categories of terrestrial ecosystems (forest, grassland, agro-ecosystem, and desert) and four of aquatic ecosystems (stream, lake, pelagic ocean and benthic ocean). We considered all ecosystems (if available) in five major global climatic zones (arctic/alpine, boreal, temperate, tropical and arid). Extended Data Table 1 provides the definitions of ecosystem categories and climatic zones. For marine ecosystems, we grouped arctic, boreal, temperate versus arid and tropical climates into "Cold" and "Warm" waters respectively, to account for a lesser influence of climate on oceanic systems due to the buffering effect of large water volumes. For each relevant ecosystem x climatic zone combination (see Extended Data Figure 2), we collected local carbon flux and spatial carbon flow data. We used all possible combinations of these categories and terms with similar meanings (see Extended Data Table 1) in our systematic search (see details in the next paragraphs).

We collected available values of subsidies linking the above-mentioned different ecosystems, and which could be converted into $\mathrm{gC} \mathrm{m}^{-2} \mathrm{yr}^{-1}$ in order to homogenize data and make comparisons possible. The latter constraint excluded cross-ecosystem flows of nutrients for which no carbon equivalent was possible, and flows expressed without information of the area of influence in the ecosystem receiving the subsidy. For instance, measurements of amount of dissolved organic carbon flowing from streams into estuaries $^{31}$ where excluded because the impacted area was undefined. Terms primarily used for the search of spatial flows were "(subsid* OR spatial flow*) AND ecosystem", with "ecosystem" also being replaced by specific ecosystems or pairwise combinations of the ecosystem types of interest. 
In addition, for each ecosystem x climatic zone combination, we systematically searched published literature for values of the following within-ecosystem carbon fluxes: gross primary production (GPP), secondary production in aquatic ecosystems, ecosystem respiration $\left(\mathrm{R}_{\mathrm{e}}\right)$, net ecosystem production (NEP), and decomposition fluxes. Since decomposition fluxes were rarely directly provided, we derived them from detritus stocks and decomposition rates (see next section for calculations). A first systematic search was conducted by using all possible combinations of the names of each ecosystem type, climatic zone and flux of interest, with small variation when relevant (e.g. "decomposition OR decay" for decomposition flux and rates). The different terminologies used across various research fields to describe the same processes, and the fact that the data of interest were often located in different sections of the studies (Methods versus Results) limited the efficiency of standardized keyword search across the data types. We therefore complemented the dataset with multiple customized searches until we compiled a minimum number of ten independent values of each variable of interest (i.e. fluxes, detritus stock, and decomposition rate) for each ecosystem x climatic zone combination. At the end, data were pooled by ecosystem type.

In total, we collected 3,034 values from 557 published studies, including 518 values of cross-ecosystem subsidies. A summary of all values and the respective references are provided in Extended data Table 2 (cross-ecosystem subsidies) and Extended data Table 3 (local fluxes).

\section{Calculations used for data extraction}

When only two of three major fluxes (production and respiration, and net ecosystem production $\left(G P P, R_{e}\right.$, and $N E P$, respectively) were reported, we estimated the unreported flux:

$$
\begin{array}{ll}
N E P=G P P-R_{e} & {[1]} \\
N E P=N P P-R_{h} & {[2]} \\
N P P=G P P-R_{a} & {[3]}
\end{array}
$$

$N P P$ is the net primary production, $R_{h}$ the heterotrophic respiration and $R_{a}$ the autotrophic respiration. 
We derived decomposition fluxes $D_{F}$ from detritus stocks $D_{M}$ and decomposition rates $k$, with the classical exponential decay model:

$$
D_{F}=D_{M}\left(1-e^{-k t}\right)
$$

To calculate individual decomposition flux values, we parameterized detritus stock $D_{M}$ with the median values of all detritus stocks in a given ecosystem $\mathrm{x}$ climatic zone combination and used the decomposition rate values collected from the literature to produce flux values. The rates were values of $k$, the first order constant in the classical exponential decay model. When not directly provided, we derived $k$ with one of the equations proposed by Cebrian and Lartigue ${ }^{14}$ depending on the data available in the study:

$$
\begin{gathered}
D_{t}=D_{t_{0}} e^{-k\left(t-t_{0}\right)} \\
D_{F}=\left(D_{P}-E\right)\left(1-e^{-k t}\right)
\end{gathered}
$$

In Equation [5], $D_{t}$ is the detrital mass at time $t$ and $D_{t_{0}}$ the initial detrital mass. This equation was used when decomposition was estimated as the proportion of detrital mass loss $\left(1-D_{t} / D_{t_{0}}\right)$ via a litter-bag experiment, a classical method in freshwater and terrestrial ecology. In equation [6], $D_{F}$ is the (absolute) decomposition flux during the study period $t$, that is the flux from detritus stock to bacteria and other detritivores, $D_{P}$ is the detritus production, and $E$ the detritus export (e.g. sedimentation). In few cases of ocean pelagic data, we used the microbial loop of primary production versus bacterial production to parameterize $D_{P}$ and $D_{F}$, respectively. If not available, the export rate was set to 0 , leading to $k$ underestimation, which is conservative in our cross-ecosystem comparison given that $k$ is already at the higher end of the range in these pelagic systems.

\section{Unit conversions}

Once collected, we standardized values by converting them all into areal carbon units, that is, $\mathrm{gC} \mathrm{m}^{-2}$ for detritus stocks and $\mathrm{gC} \mathrm{m}^{-2} \mathrm{yr}^{-1}$ for local fluxes and cross-ecosystem subsidies. Decomposition rates were expressed in $\mathrm{yr}^{-1}$.

Carbon conversion: We used data in carbon units $(\mathrm{gC})$ when it was directly provided in the study, or we calculate the values using carbon content when reported in the study. Alternatively, we applied the most direct conversion of the data into carbon 
units depending on the level of detail available (see Extended data Table 4 for conversion factors). For decomposition rates, we did not transform units into carbon, assuming carbon loss rate to be identical to loss rate in the unit provided.

Time extrapolation: for local fluxes or rates provided in daily units, we extrapolated to the year by multiplying by 365 days in tropical systems where low or no seasonality is assumed. For the others, we multiplied by the number of days in the growing season as reported in the study, or the ice-free period in cold climates. When growing season length (GSL) was not specified in the study we used averaged estimates detailed by Garonna et al. ${ }^{32}$ for the different climatic zones in Europe $^{33}: 181$ days for temperate climate (mean of atlantic and continental), 155 days for boreal, 116 days for arctic, and 163 days for arid systems (mean of Mediterranean and steppic). We did not apply any conversion if the value was measured on a study period longer than the above GSL for the corresponding climate.

Volume to area conversions and depth integration: Some data were given per unit of volume. For freshwater systems, we converted the data into area units by integrating them over the water column, using the mean depth of the river or lake. When not directly available in the study we calculated depth by dividing the volume per the area in lakes, or by estimating depth from discharge in rivers with the formula depth $=c \cdot Q^{f}$, with $c=0.2, f=0.4$ and $Q$ the discharge in $\mathrm{m}^{3} \mathrm{~s}^{-1} 34$. For small catchment areas, that is $<1$ $\mathrm{km}^{2}$, we estimated the depth to be $5 \mathrm{~cm}$ based on known river scaling-properties ${ }^{34}$. For marine data, notably production in the pelagic zone, studies generally provide a meaningful depth, which defines the euphotic zone such as the Secchi depth or the $1 \%$ light inflow depth. We integrated values in volume units over this depth, and to $100 \mathrm{~m}$ depth when only sampling depths were provided.

Areal units for cross-ecosystem subsidies: Since we were interested in comparing the magnitude of spatial subsidies to that of the recipient ecosystem's own local fluxes, we needed cross-ecosystem subsidies measured in areal units of the recipient ecosystem. Depending on the method of quantification, cross-ecosystem flow can be directly expressed in this way (e.g. litter traps in the recipient ecosystem). In other cases, for example with vertical fluxes between pelagic and benthic ecosystems, the equivalence of donor and recipient ecosystem areas affected by the flow is obvious. However, in the case 
of lateral flows occurring from aquatic to terrestrial ecosystems (e.g. emergent insects, carcasses of salmon caught by bears), flows were often provided per $\mathrm{m}^{2}$ of donor area. We could not use such measurements directly because the magnitude of the flow in the recipient ecosystem depends on both the total surface of production and the boundary length. For instance, lakes with the same area but having circular versus complexshorelines will lead, for the same total emergent insect flux, to higher versus lower magnitudes of flows, respectively, distributed per areal unit of the recipient ecosystem. Moreover, the maximum influence of such cross-ecosystem flow is found near at the shoreline and decreases with distance from the shore (for example, in aquatic insects ${ }^{19}$ ). To adjust for this in a conservative approach, we homogenized our aquatic-to-terrestrial flows assuming a uniform distribution of the flow on the first ten meters from the shore, a distance within which most of the aquatic insect flows fall ${ }^{35}$ (but could fall substantially farther in some specific systems ${ }^{36}$ ), or most of the salmon carcasses brought by bears on land are deposited ${ }^{37}$. Therefore, when the values of spatial flows were provided in areal unit of the donor ecosystem (aquatic), we first calculated the flow per meter shoreline (in some cases, values were already provided per meter shoreline; e.g., wrack deposited on beaches), and then divided this value by 10 meters of influenced land. To calculate the spatial flow per shoreline length in freshwater systems, we followed the method described by Gratton et al. ${ }^{19}$ for insect emergence data: In lakes we multiplied the data per the total lake area and divided per the perimeter. When not directly available, the perimeter was approximated by $2 * D_{L} *(\operatorname{area} * \pi)^{1 / 2}$, with $D_{L}$ the development factor defined by $\mathrm{Kalff}^{38}$, which is 1 for circular shapes, 2 for the same area with a twofold larger perimeter. In streams we multiplied the original donor area flow value by stream width, and then divided it per two (riversides) to obtain the flow per shoreline length. The Extended Data Figure S3 show that our conclusions are not sensitive to the choice of the distance from shoreline used to calculate the recipient terrestrial area of aquatic subsidies: using a more conservative threshold of $100 \mathrm{~m}$ only decreases the importance of spatial subsidies for terrestrial ecosystems, that we already assess as low. 


\section{Flow drivers}

We defined categories of flow drivers to examine the underlying processes of documented cross-ecosystem subsidies. Drivers could be either passive, via physical processes such as gravity, wind, water currents, tides, diffusion, or active, via animal movements such as those triggered by foraging behaviours, seasonal migration or crossecosystem movement of animals needed to complete a life-cycle. We kept these last three categories for active drivers and we clumped the passive drivers into categories reflecting broad classes of spatial flows: "Fall/wind" for aerial transport from terrestrial systems, "leaching" for diffusion processes, "current, tides" for lateral flows from aquatic systems and "sinking" for vertical passive flows in the water column (see Extended data Table 5). We analysed the data and plotted the figures with the software $\mathrm{R}^{39}$ and the R-packages $\operatorname{ggmap}^{40}$, maps $^{41}$, and pgirmess ${ }^{42}$.

\section{Data availability}

Data that support the findings of this study are summarized in Extended Data Table 2 and Extended Data Table 3, with all associated references in Supplementary Table 1. 


\section{References}

1. Polis, G. A., Power, M. E. \& Huxel editors, G. R. Food Webs at the landscape level. (University of Chicago Press, Chicago and London, 2004).

2. Richardson, J. S. \& Sato, T. Resource subsidy flows across freshwater-terrestrial boundaries and influence on processes linking adjacent ecosystems. Ecohydrology 8, 406-415 (2015).

3. Leroux, S. J. \& Loreau, M. Dynamics of Reciprocal Pulsed Subsidies in Local and Meta-Ecosystems. Ecosystems 15, 48-59 (2012).

4. Gravel, D., Guichard, F., Loreau, M. \& Mouquet, N. Source and sink dynamics in meta-ecosystems. Ecology 91, 2172-2184 (2010).

5. Gounand, I. et al. The Paradox of Enrichment in Metaecosystems. Am. Nat. 184, 752-763 (2014).

6. Gounand, I., Harvey, E., Ganesanandamoorthy, P. \& Altermatt, F. Subsidies mediate interactions between communities across space. Oikos 126, 972-979 (2017).

7. Bultman, H., Hoekman, D., Dreyer, J. \& Gratton, C. Terrestrial deposition of aquatic insects increases plant quality for insect herbivores and herbivore density. Ecol.Entomol.39, 419-426 (2014).

8. Schindler, D. E. \& Smits, A. P. Subsidies of Aquatic Resources in Terrestrial Ecosystems. Ecosystems 20, 78-93 (2017).

9. Gounand, I., Harvey, E., Little, C. J. \& Altermatt, F. Meta-Ecosystems 2.0 :

Rooting the Theory into the Field. Trends Ecol. Evol. 33, 36-46 (2018).

10. Millenium Ecosystem Assessment Panel. Ecosystems and Human Well-Being: Synthesis. (Island Press Washington DC, 2005).

11. Russell, R. et al. Humans and Nature : How Knowing and Experiencing Nature Affect Well-Being. Annu. Rev. Environ. Resour. 38, 473-502 (2013).

12. Vörösmarty, C. J. et al. Global threats to human water security and river biodiversity. Nature 467, 555-561 (2010).

13. Halpern, B. S., Walbridge, S., Al, E. \& Wtsaon, R. A global map of human impact on marine ecosystems. Science. 319, 948-952 (2008).

14. Cebrian, J. \& Lartigue, J. Patterns of herbivory and decomposition in aquatic and 
terrestrial ecosystems. Ecol. Monogr. 74, 237-259 (2004).

15. Leibold, M. A. et al. The metacommunity concept: a framework for multi-scale community ecology. Ecol. Lett. 7, 601-613 (2004).

16. Massol, F. et al. How life-history traits affect ecosystem properties: effects of dispersal in meta-ecosystems. Oikos 126, 532-546 (2017).

17. Reiners, W. A. \& Driese, K. Transport of energy, information and material through the biosphere. Annu. Rev. Environ. Resour. 28, 107-135 (2003).

18. Loreau, M., Mouquet, N. \& Holt, R. D. Meta-ecosystems: a theoretical framework for a spatial ecosystem ecology. Ecol. Lett. 6, 673-679 (2003).

19. Gratton, C. \& Vander Zanden, M. J. Flux of aquatic insect productivity to land: Comparison of lentic and lotic ecosystems. Ecology 90, 2689-2699 (2009).

20. Greig, H. S. et al. Warming, eutrophication, and predator loss amplify subsidies between aquatic and terrestrial ecosystems. Glob. Chang. Biol. 18, 504-514 (2012).

21. Ball, B. a. et al. Direct and Terrestrial Vegetation-mediated Effects of Environmental Change on Aquatic Ecosystem Processes. Bioscience 60, 590-601 (2010).

22. Griffiths, J. R. et al. The importance of benthic-pelagic coupling for marine ecosystem functioning in a changing world. Glob. Chang. Biol. 23, 2179-2196 (2017).

23. Elser, J. J. et al. Nutritional constraints in terrestrial and freshwater food webs. Nature 408, 578-80 (2000).

24. Adame, M., Fry, B., Gamboa, J. \& Herrera-Silveira, J. Nutrient subsidies delivered by seabirds to mangrove islands. Mar. Ecol. Prog. Ser. 525, 15-24 (2015).

25. Doughty, C. E. et al. Global nutrient transport in a world of giants. Proc. Natl. Acad. Sci.113, 1-6 (2015).

26. Soininen, J., Bartels, P., Heino, J., Luoto, M. \& Hillebrand, H. Toward More Integrated Ecosystem Research in Aquatic and Terrestrial Environments. Bioscience 65, 174-182 (2015).

27. Abbas, F. et al. Roe deer may markedly alter forest nitrogen and phosphorus budgets across Europe. Oikos 121, 1271-1278 (2012). 
28. Bauer, S. \& Hoye, B. J. Migratory animals couple biodiversity and ecosystem functioning worldwide. Science. 344, 1242552 (2014).

29. Lundberg, J. \& Moberg, F. Mobile link organisms and ecosystem functioning: Implications for ecosystem resilience and management. Ecosystems 6, 87-98 (2003).

30. Baxter, C. V., Fausch, K. D. \& Saunders, W. C. Tangled webs: Reciprocal flows of invertebrate prey link streams and riparian zones. Freshw. Biol. 50, 201-220 (2005).

31. Regnier, P. et al. Anthropogenic perturbation of the carbon fluxes from land to ocean. Nat. Geosci. 6, 597 (2013).

32. Garonna, I. et al. Strong contribution of autumn phenology to changes in satellitederived growing season length estimates across Europe (1982-2011). Glob. Chang. Biol. 20, 3457-3470 (2014).

33. Mücher, C. A., Klijn, J. A., Wascher, D. M. \& Schaminée, J. H. J. A new European Landscape Classification (LANMAP): A transparent, flexible and useroriented methodology to distinguish landscapes. Ecol. Indic. 10, 87-103 (2010).

34. Rodriguez-Iturbe, I. \& Rinaldo, A. Fractal river networks: chance and selforganization. (Cambridge University Press, 1997).

35. Iwata, T., Nakano, S. \& Murakami, M. Stream meanders increase insectivorous bird abundance in riparian deciduous forests. Ecography. 26, 325-337 (2003).

36. Gratton, C., Donaldson, J. \& Zanden, M. J. Vander. Ecosystem Linkages Between Lakes and the Surrounding Terrestrial Landscape in Northeast Iceland. Ecosystems 11, 764-774 (2008).

37. Gende, S. M., Quinn, T. P., Willson, M. F., Heintz, R. \& Scott, T. M. Magnitude and fate of salmon-derived nutrients and energy in a coastal stream ecosystem. $J$. Freshw. Ecol. 19, 149-160 (2004).

38. Kalff, J. Limnology-Inland water ecosystems. (Upper Saddle River, N.J. : Prentice Hall, 2002). doi:10.2307/1468422

39. R Core Team. R: A Language and Environment for Statistical Computing. (R Foundation for Statistical Computing, Vienna, Austria. URL https://www.Rproject.org/, 2017). 
40. Kahle, D. \& Wickham, H. ggmap: Spatial Visualization with ggplot2. The $R$ Journal. 5, 144-161 (2013).

41. Original S code by Becker, R. A., and Wilks, A. R., R version by Brownrigg, R., Enhancements by Minka, T. P. \& and Deckmyn, A. maps: Draw Geographical Maps. $R$ Packag. version 3.2.0. URL https//cran.r-project.org/package=maps (2017).

42. Giraudoux, P. pgirmess: Data Analysis in Ecology. R Packag. version 1.6.6. URL https//CRAN.R-project.org/package=pgirmess (2017). 


\section{Acknowledgements}

We thank Marcel Holyoak and Emanuel A. Fronhofer for discussions, and Mary

O'Connor for comments on the manuscript.

\section{Author contribution}

I.G., C.J.L., E.H. and F.A. conceived the study and collected the data. I.G. analysed the data, produced the figures and wrote the first draft of the manuscript. All authors edited and contributed to the final version of the manuscript. 


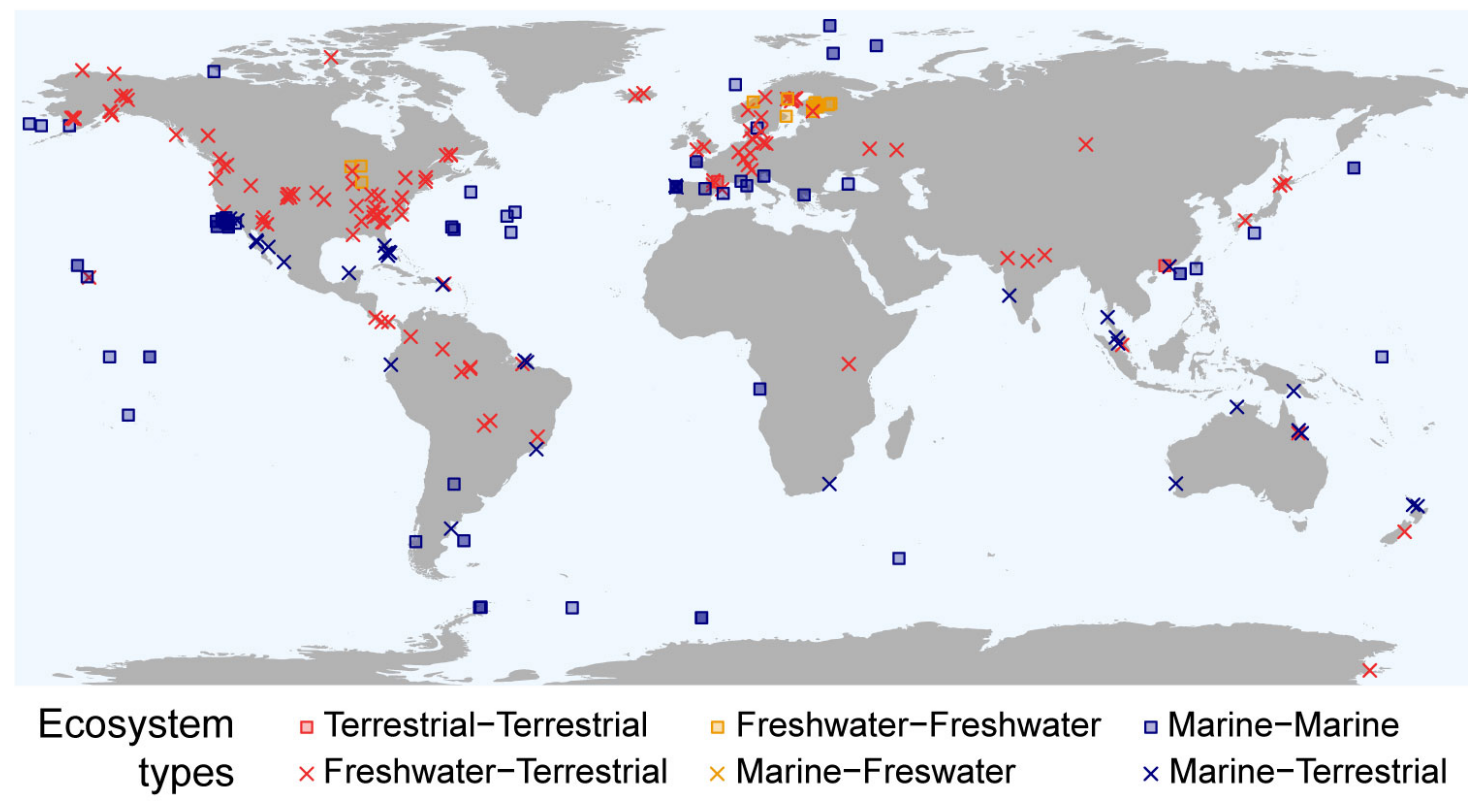

Figure 1 I Global distribution of available cross-ecosystem subsidy data. Colours and shapes indicate the type of ecosystems coupled by cross-ecosystem subsidies: terrestrial (i.e., forest, grassland, agro-ecosystem, desert), marine (i.e., ocean pelagic, ocean benthic) and freshwater (i.e., stream, lake, wetland). 

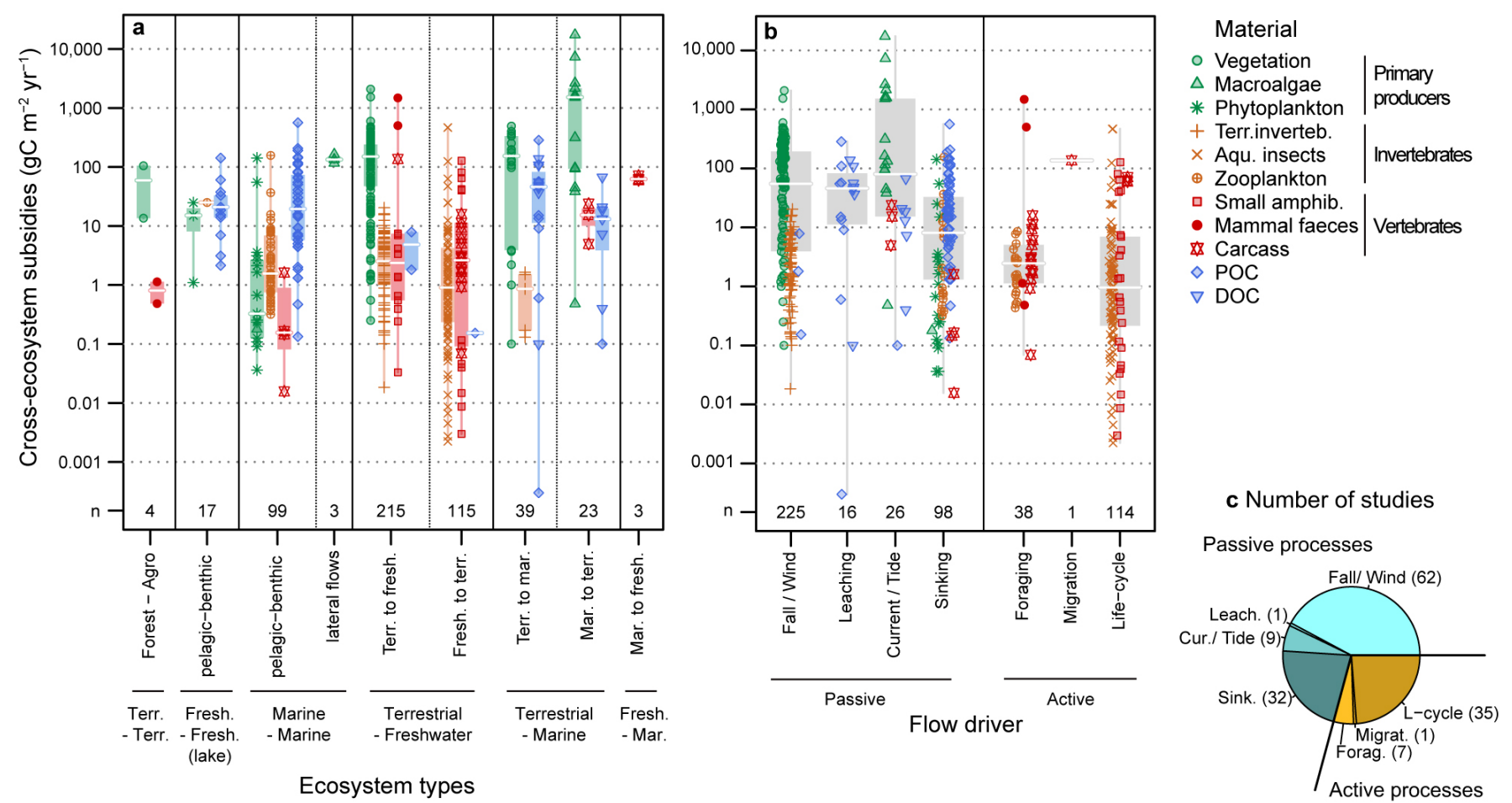

Figure 2 I Architecture of cross-ecosystem carbon subsidies. Values are provided (a)

by types of ecosystems connected by the subsidies, with vertical labels specifying ecosystems (“Agro" stands for “Agro-ecosystem”) or flow direction, and (b) by underlying flow driver, either via passive or active processes. Shapes and colours denote material transported. $\mathbf{a}, \mathbf{b}$ - Bottom numbers, $n$, indicate the number of data points.

Values are in $\mathrm{gC} \mathrm{m}^{-2} \mathrm{y}^{-1}$ on a $\log$ scale. The areal unit, $\mathrm{m}^{2}$, refers to the recipient ecosystem. Boxplots give median (white line), $25 \%$ and $75 \%$ percentiles (box), and range (whiskers). c - Number of studies by driver type. 


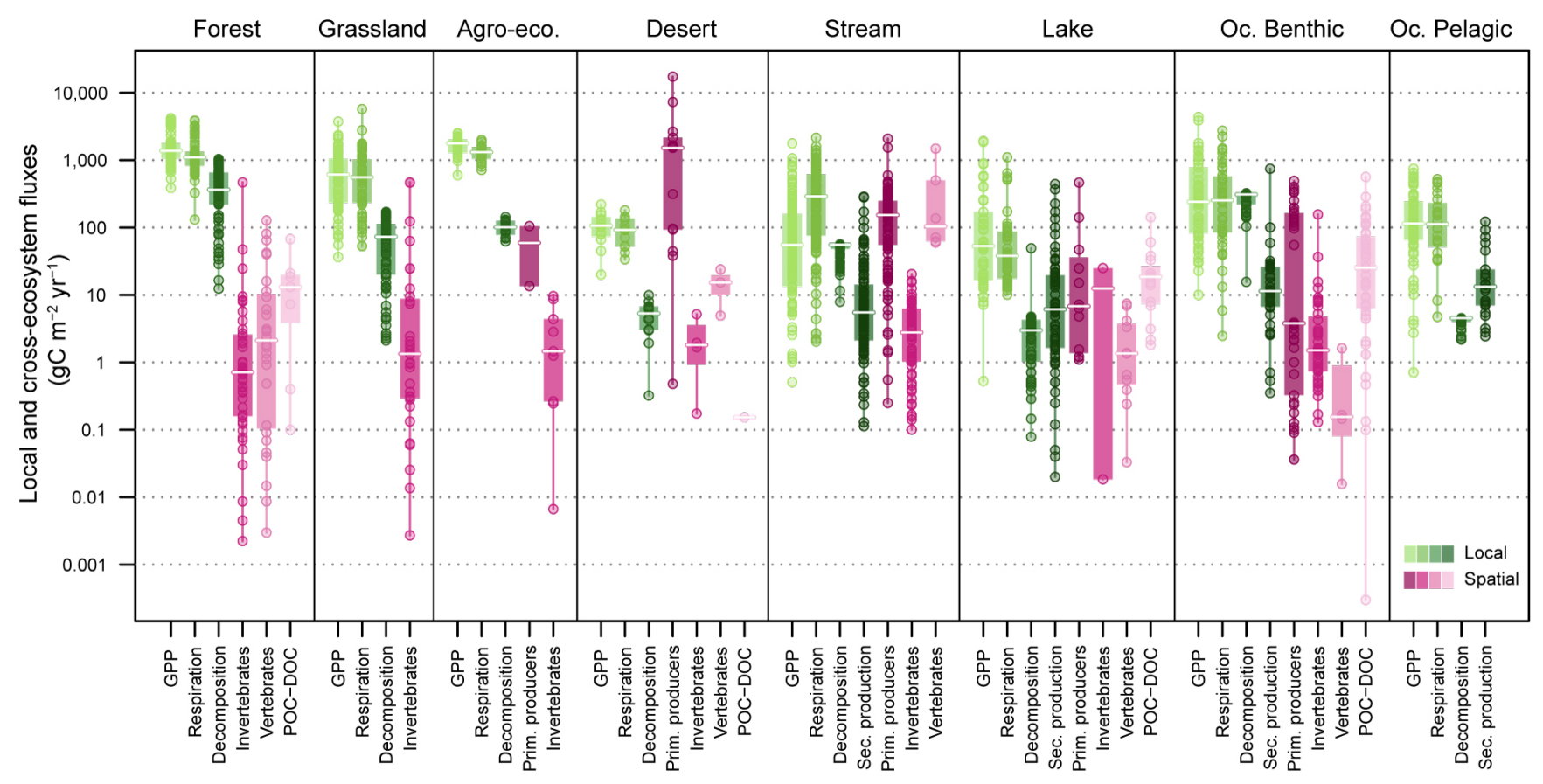

Figure 3 I Comparison of local fluxes versus cross-ecosystem subsidies. Local fluxes within (green), and cross-ecosystem subsidies to (pink), specific ecosystem types (different panels). Represented local fluxes are gross primary production, ecosystem respiration, decomposition flux, and secondary production in aquatic systems. Crossecosystem subsidies are clumped into imported material of four origins: primary producers invertebrates, vertebrates, and organic carbon (particulate and dissolved). No subsidy to pelagic systems was documented. Circles give values in $\mathrm{gC} \mathrm{m}^{-2} \mathrm{y}^{-1}$ on a $\log$ scale. Boxplots give median (white line), $25 \%$ and $75 \%$ percentiles (box), and range (whiskers). Whisker and three null values of GPP in streams are omitted due to log scale. 
a Terrestrial - Freshwater coupling

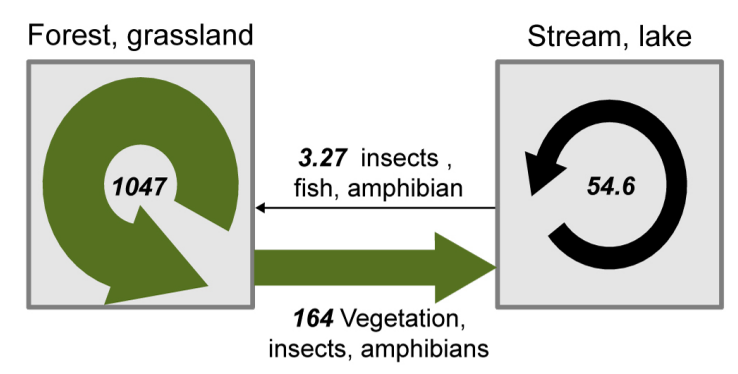

b Pelagic - Benthic coupling

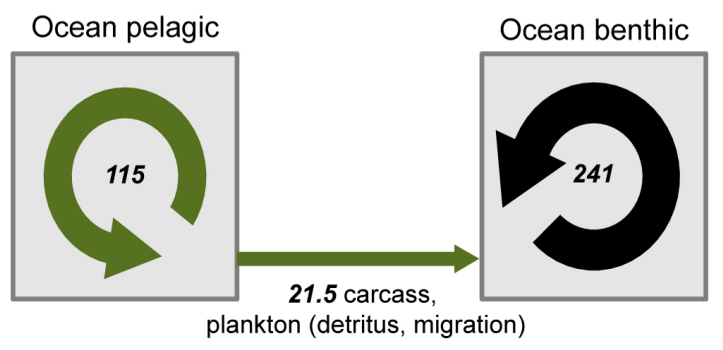

Figure 4 I Well-documented natural meta-ecosystems. Cross-ecosystem subsidies suggest significant spatial couplings between (a) terrestrial and freshwater ecosystems and (b) pelagic and benthic areas in marine ecosystems. Round and horizontal arrows represent gross primary production (GPP) and cross-ecosystem subsidies, respectively. Numbers in italic are median values for GPP and sum of median values of crossecosystem subsidies of each different origin (primary producer, invertebrate, vertebrate, and particulate and dissolved organic carbon), both expressed in $\mathrm{gC} \mathrm{m}^{-2} \mathrm{y}^{-1}$. Width of arrows is proportional to these values (based on double squared root transformation). 


\section{Extended Data Display}

\section{Worldwide cross-ecosystem carbon subsidies and their contribution to ecosystem functioning}

Isabelle Gounand, Chelsea J. Little, Eric Harvey, and Florian Altermatt

Corresponding author: Isabelle Gounand - isabelle.gounand@ eawag.ch

\section{Content}

Extended Data Figure 1: Net Ecosystem Production for different ecosystem types............... 24

Extended Data Figure 2: Data collection................................................ 25

Extended Data Figure 3: Sensitivity of local fluxes versus cross-ecosystem subsidies to

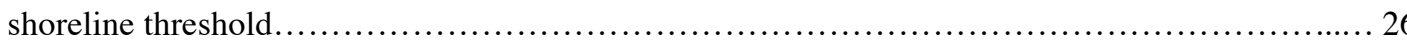

Extended Data Table 1: Definition of ecosystem and climate categories........................ 27

Extended Data Table 2: Summary of cross-ecosystem subsidy data........................... 28

Extended Data Table 3: Summary of local ecosystem data............................... 29

Extended Data Table 4: Factors used for conversions into grams of carbon....................... 31

Extended Data Table 5: Definition of flow drivers....................................... 32 


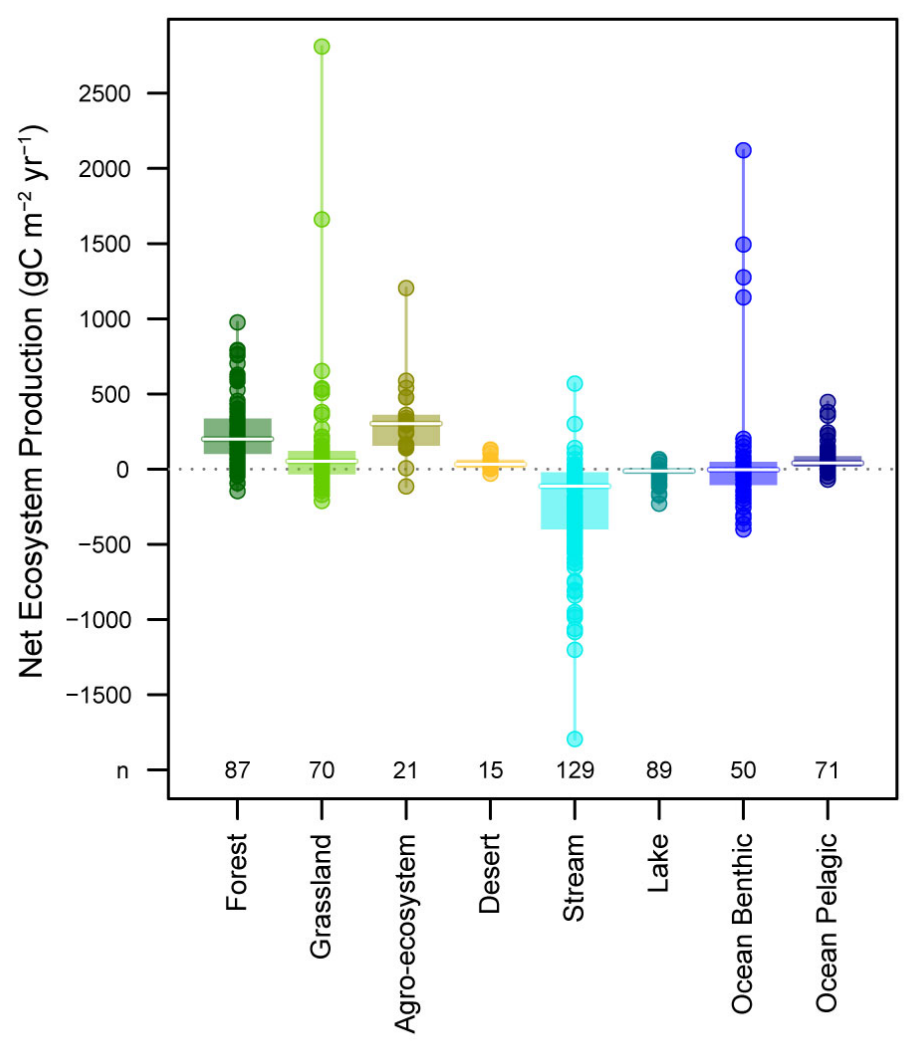

\section{Extended Data Figure 1 I Net Ecosystem Production for different ecosystem types.}

Net ecosystem production corresponds to the balance between gross primary production and ecosystem respiration. Negative values denote net heterotrophic functioning. Circles give individual values in $\mathrm{gC} \mathrm{m}^{-2} \mathrm{y}^{-1}$. Note that these are production fluxes and not productivity rates (biomass turnover), for which we would have higher values in aquatic compared to terrestrial systems. Boxplots give median (white line), 25\% and 75\% percentiles (box), and range (whiskers). Bottom numbers (n) indicate the number of data points. 


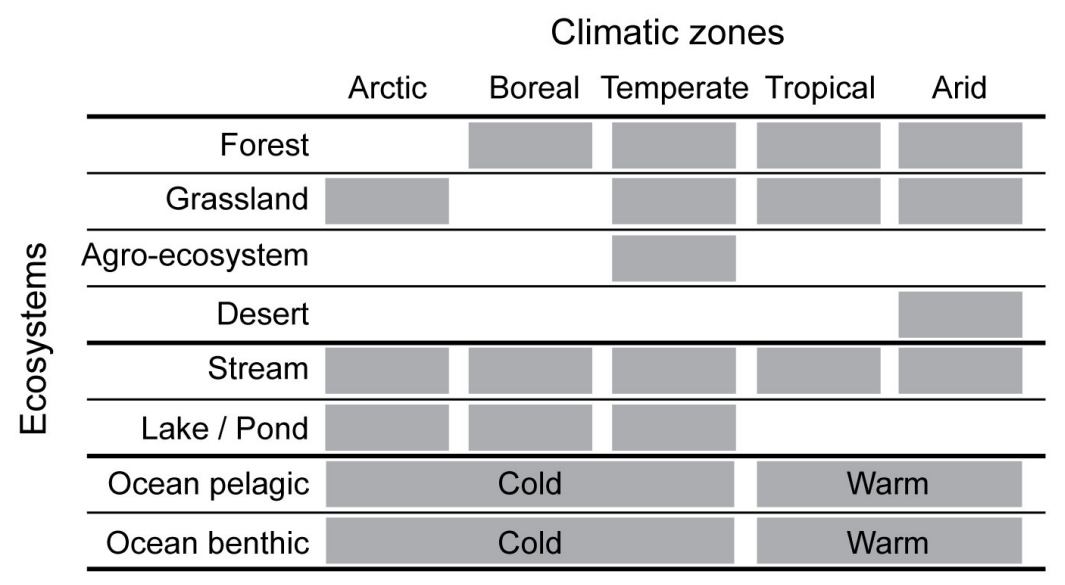

Extended Data Figure 2 I Data collection. Combinations of ecosystem types and climatic zones considered in this study. We collected values of local carbon fluxes for all combinations marked by a grey rectangle, as well as cross-ecosystem subsidies linking these ecosystems. The other combinations either do not exist, or do not have enough data available. 


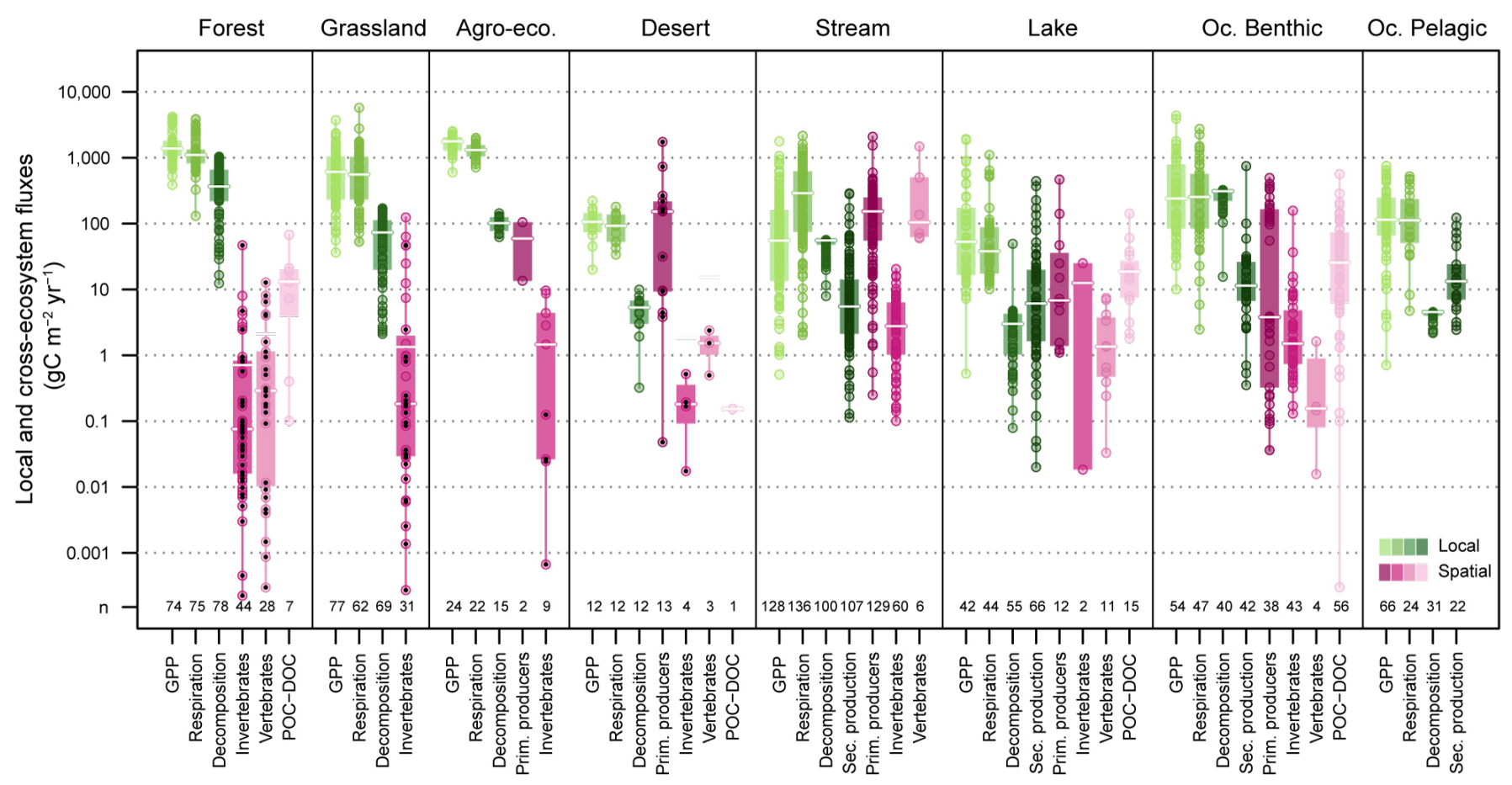

\section{Extended Data Figure 3 I Sensitivity of local fluxes versus cross-ecosystem subsidies}

to shoreline threshold. Local fluxes within- (green) and cross-ecosystem subsidies to(pink), specific ecosystem types (different panels). A threshold of 100 meters from shoreline (rather than $10 \mathrm{~m}$ in Figure 3) was used to calculate the recipient terrestrial area influenced by aquatic cross-ecosystem subsidies. Circles give values in $\mathrm{gC} \mathrm{m}^{-2} \mathrm{y}^{-1}$ on a log scale. Black dots show the data points influenced by this choice. Boxplots give median (white line), $25 \%$ and $75 \%$ percentiles (box), and range (whiskers). Whisker and three null values of GPP in streams are omitted due to log scale. Bottom numbers (n) indicate the number of data points. 
Extended Data Table 1 I Definition of ecosystem and climate categories.

\begin{tabular}{|c|c|c|}
\hline & Definition & Example ecosystems \\
\hline \multicolumn{3}{|l|}{ Climatic zones } \\
\hline Arctic & $\begin{array}{l}\text { Extreme temperature limitation of growing season length, } \\
\text { with abiotic conditions not supporting tree growth in arctic, } \\
\text { subarctic, and alpine zones; OR high latitude oceans, } \\
\text { generally above } 66.5^{\circ}\end{array}$ & $\begin{array}{l}\text { Tundra (grassland) } \\
\text { Alpine grassland }\end{array}$ \\
\hline Boreal & $\begin{array}{l}\text { Strong temperature limitation of terrestrial growth, but } \\
\text { environment supports tree growth. Covers northern parts of } \\
\text { North America, Europe and Russia from latitudes } 50^{\circ} \text { to } \\
55^{\circ} \text {; OR oceans between } 50^{\circ} \text { and } 66.5^{\circ}\end{array}$ & $\begin{array}{l}\text { Taiga (forest) } \\
\text { Sub-alpine forest }\end{array}$ \\
\hline & Seasonal terrestrial growth with some temperature & \\
\hline Temperate & $\begin{array}{l}\text { limitation. Covers latitudes between } 23.5^{\circ} \text { and } 50^{\circ} \text { to } 55^{\circ} \text {, } \\
\text { including oceans in this latitudinal range }\end{array}$ & Beech forest \\
\hline Tropical & $\begin{array}{l}\text { Warm terrestrial tropical, sub-tropical, equatorial systems } \\
\text { not limited by drought between } 0^{\circ} \text { to } 23.5^{\circ} \text { latitude } \\
\text { (including subtropical system), including oceans in this } \\
\text { latitude range }\end{array}$ & Savanna (grassland), rainforest \\
\hline Arid & $\begin{array}{l}\text { Severely water-limited terrestrial systems at all latitudes, } \\
\text { including arid, semi-arid, xeric, xerophytic, xeromorphic, } \\
\text { Mediterranean systems, continental, warm or cold, and } \\
\text { polar deserts }\end{array}$ & $\begin{array}{l}\text { Garrigue (grassland), shrubland } \\
\text { (grassland or forest, depending on the } \\
\text { canopy), chaparral (grassland), steppe } \\
\text { (grassland), caatinga (forest), cerrado }\end{array}$ \\
\hline \multicolumn{3}{|l|}{ Ecosystems } \\
\hline Forest & $\begin{array}{l}\text { Complete vegetation cover with trees as dominant } \\
\text { vegetation; tree canopy covers most of the surface }\end{array}$ & $\begin{array}{l}\text { Rainforest, caatinga, woodland, some } \\
\text { shrubland, cerrado }\end{array}$ \\
\hline Grassland & $\begin{array}{l}\text { Complete vegetation cover, but with only very few or no } \\
\text { trees; vegetation dynamics dominated by water limitation, } \\
\text { fires and grazing. }\end{array}$ & $\begin{array}{l}\text { Steppe, savannah, meadow, prairie, } \\
\text { tundra, old field, some shrublands, } \\
\text { herbaceous rich-fen vegetation. }\end{array}$ \\
\hline Desert & $\begin{array}{l}\text { Extreme growth limitation by water availability, with little } \\
\text { vegetation distributed in remote patches }\end{array}$ & Sandy land. \\
\hline Agro-ecosystem & $\begin{array}{l}\text { Ecosystems devoted to crop production or cattle grazing, } \\
\text { often fertilized or irrigated to remove nutrient or water } \\
\text { limitations for growth }\end{array}$ & Cropland, pasture. \\
\hline Stream & $\begin{array}{l}\text { Running freshwater and lotic systems of all sizes, including } \\
\text { rivers }\end{array}$ & Creek, brook, river, stream \\
\hline Lake & Standing (lentic) freshwater systems & Reservoir, lake, pond \\
\hline Ocean & $\begin{array}{l}\text { All salt water ecosystems with no emerged vegetation, } \\
\text { including internal seas }\end{array}$ & Sea, ocean shelf, estuary, lagoon \\
\hline Ocean pelagic & Ecosystems in the open water columns of oceans and seas & Upwelling system, open ocean \\
\hline Ocean benthic & Ecosystems at the bottom of oceans and seas & $\begin{array}{l}\text { Coral reef, sea grass bed, eelgrass } \\
\text { meadow, kelp forest, deep-sea flour. }\end{array}$ \\
\hline
\end{tabular}


Extended Data Table 2 I Summary of cross-ecosystem subsidy data.

\begin{tabular}{|c|c|c|c|c|c|c|c|c|c|}
\hline Donor & Recipient & Material & $\mathrm{n}$ & Min & q25 & Median & $\mathrm{q} 75$ & Max & Data References \\
\hline Forest & Agroeco. & Vegetation & 2 & 13.545 & 36.360 & 59.175 & 81.990 & 104.81 & 50 \\
\hline Forest & Stream & Vegetation & 96 & 0.2500 & 55.247 & 178.65 & 280.62 & $2,085.8$ & $\begin{array}{l}8,12,13,14,15,16,17,21,26,27,28,29,30,31,33,65,78,90,96,117,118 \\
123,124,138,203,205,212,235,375,389,392\end{array}$ \\
\hline Forest & Stream & Terr.inverteb. & 49 & 0.1414 & 1.0683 & 3.0368 & 7.3321 & 20.386 & $2,3,4,5,6,7,23,25,32,65,98,147,365,369,370,375,525,526$ \\
\hline Forest & Lake & Vegetation & 9 & 1.2000 & 1.5439 & 6.4000 & 47.000 & 465.00 & $20,109,295,316,375,520,521$ \\
\hline Forest & Lake & Terr.inverteb. & 1 & 0.0184 & 0.0184 & 0.0184 & 0.0184 & 0.0184 & 375 \\
\hline Forest & Lake & Small amphib. & 11 & 0.0331 & 0.4706 & 1.3528 & 3.7450 & 7.4100 & 60,61 \\
\hline Forest & Lake & POC & 2 & 1.8100 & 3.3259 & 4.8418 & 6.3576 & 7.8735 & 522 \\
\hline Forest & Ocean & Vegetation & 18 & 0.1000 & 27.249 & 153.80 & 312.00 & 492.80 & 430,523 \\
\hline Forest & Ocean & Terr.inverteb. & 5 & 0.1300 & 0.1700 & 0.8600 & 1.5100 & 1.6400 & 527 \\
\hline Forest & Ocean & POC & 9 & 0.0003 & 9.1000 & 15.100 & 56.900 & 285.00 & 430 \\
\hline Forest & Ocean & DOC & 7 & 0.1000 & 40.500 & 48.000 & 82.050 & 138.10 & 430 \\
\hline Grassland & Stream & Vegetation & 9 & 0.5500 & 16.000 & 64.593 & 250.00 & 265.77 & $16,18,65,89,117,118,138,205$ \\
\hline Grassland & Stream & Terr.inverteb. & 6 & 0.1005 & 0.3413 & 0.8304 & 2.0334 & 2.5296 & $9,32,365$ \\
\hline Grassland & Stream & Mammal faeces & 2 & 502.00 & 746.75 & 991.50 & $1,236.2$ & $1,481.0$ & 364 \\
\hline Grassland & Stream & Carcass & 1 & 136.80 & 136.80 & 136.80 & 136.80 & 136.80 & 556 \\
\hline Agroeco. & Forest & Mammal faeces & 2 & 0.4829 & 0.6448 & 0.8067 & 0.9686 & 1.1305 & 367 \\
\hline Agroeco. & Stream & Vegetation & 23 & 1.4000 & 61.814 & 104.70 & 165.15 & 264.80 & 12,78 \\
\hline Agroeco. & Stream & Terr.inverteb. & 5 & 1.4544 & 4.4640 & 5.2699 & 6.1504 & 6.1504 & $3,98,365$ \\
\hline Desert & Stream & Vegetation & 1 & 8.4000 & 8.4000 & 8.4000 & 8.4000 & 8.4000 & 94 \\
\hline Wetland & Forest & Small amphib. & 1 & 64.634 & 64.634 & 64.634 & 64.634 & 64.634 & 59 \\
\hline Wetland & Grassland & Aqu. insects & 6 & 0.0136 & 0.0339 & 0.1410 & 0.3297 & 2.4408 & 57,66 \\
\hline Stream & Forest & Aqu. insects & 30 & 0.0022 & 0.1377 & 0.4857 & 0.9384 & 8.3480 & $10,11,19,65,67,74,77,78,147,266,365,366,477$ \\
\hline Stream & Forest & Carcass & 14 & 0.0693 & 1.7076 & 2.9078 & 5.8411 & 15.980 & 386,390 \\
\hline Stream & Grassland & Aqu. insects & 5 & 0.0027 & 0.0628 & 0.1329 & 0.2828 & 0.9516 & $65,77,365$ \\
\hline Stream & Agroeco. & Aqu. insects & 9 & 0.0067 & 0.2648 & 1.4607 & 4.3822 & 9.6100 & $69,77,78,365$ \\
\hline Stream & Desert & Aqu. insects & 4 & 0.1742 & 1.3000 & 1.8098 & 2.7536 & 5.1815 & $22,34,387$ \\
\hline Lake & Forest & Aqu. insects & 14 & 0.0976 & 0.7246 & 1.8110 & 9.1252 & 467.87 & $62,368,374,518,524,528$ \\
\hline Lake & Forest & Small amphib. & 11 & 0.0030 & 0.0273 & 0.0914 & 41.002 & 127.33 & $58,60,528$ \\
\hline Lake & Grassland & Aqu. insects & 20 & 0.3057 & 1.2527 & 4.7484 & 24.641 & 467.87 & $1,24,62,368,374,383,517,519$ \\
\hline Lake & Desert & POC & 1 & 0.1530 & 0.1530 & 0.1530 & 0.1530 & 0.1530 & 56 \\
\hline L. Pelagic & L. Benthic & Phytoplankton & 3 & 1.0971 & 8.1016 & 15.106 & 19.965 & 24.824 & $99,134,379$ \\
\hline L. Pelagic & L. Benthic & Zooplankton & 1 & 24.983 & 24.983 & 24.983 & 24.983 & 24.983 & 379 \\
\hline L. Pelagic & L. Benthic & POC & 13 & 2.1448 & 13.950 & 20.925 & 32.860 & 141.82 & $294,296,485$ \\
\hline Ocean & Forest & POC & 1 & 0.1000 & 0.1000 & 0.1000 & 0.1000 & 0.1000 & 430 \\
\hline Ocean & Forest & DOC & 6 & 0.4000 & 8.7500 & 15.900 & 20.350 & 67.300 & 430 \\
\hline Ocean & Desert & Macroalgae & 6 & 44.520 & 93.921 & 835.79 & $1,638.3$ & $2,646.9$ & $103,104,371$ \\
\hline Ocean & Desert & Carcass & 3 & 4.9500 & 10.125 & 15.300 & 19.575 & 23.850 & 104 \\
\hline Ocean & Stream & Carcass & 3 & 59.667 & 61.075 & 62.483 & 66.847 & 71.211 & 389 \\
\hline Oc. Pelagic & Oc. Benthic & Macroalgae & 1 & 0.1786 & 0.1786 & 0.1786 & 0.1786 & 0.1786 & 503 \\
\hline Oc. Pelagic & Oc. Benthic & Phytoplankton & 16 & 0.0362 & 0.1222 & 0.4978 & 2.7648 & 142.00 & $310,311,499,539$ \\
\hline Oc. Pelagic & Oc. Benthic & Zooplankton & 38 & 0.3167 & 0.7563 & 1.5629 & 6.4680 & 156.90 & $308,311,384,385,495,539,557$ \\
\hline Oc. Pelagic & Oc. Benthic & Carcass & 4 & 0.0157 & 0.1134 & 0.1556 & 0.5290 & 1.6200 & $499,500,503,504$ \\
\hline Oc. Pelagic & Oc. Benthic & POC & 40 & 0.1330 & 5.7875 & 19.444 & 70.250 & 562.91 & $80,102,116,131,133,304,306,311,319,330,334,335,338,393,494,514$ \\
\hline Oc. Benthic & Desert & Macroalgae & 7 & 0.4795 & 176.46 & $1,522.1$ & $4,730.8$ & 17,365 & $372,373,382$ \\
\hline Oc. Benthic & Oc. Benthic & Macroalgae & 3 & 115.80 & 124.65 & 133.50 & 148.75 & 164.00 & 418,420 \\
\hline
\end{tabular}

Agroeco.$=$ Agro-ecosystem; Oc. $=$ Ocean; Terr. $=$ Terrestrial; Aqu. $=$ Aquatic $;$ inverteb.$=$ invertebrates; amphib. $=$ amphibians $;$ POC $=$ Particulate Organic Carbon; DOC $=$ Dissolved Organic Carbon.

We provide the number of data points (n), the minimum, 25\% quartile (q25), median, 75\% quartile (q75) and maximum values of estimates distribution for each pair of ecosystems coupled and per type of the material exchanged, as well as the associated references from which we extracted the data, listed in Supplementary Table 1. Values are expressed in $\mathrm{gC} \mathrm{m}^{-2} \mathrm{yr}^{-1}$. Donor and Recipient refer respectively to the ecosystem from which comes and to which go the spatial flow. 
Extended Data Table 3 I Summary of local ecosystem data.

\begin{tabular}{|c|c|c|c|c|c|c|c|c|}
\hline Ecosystem & Variable & $\mathrm{n}$ & Min & $\mathrm{q} 25$ & Median & $\mathrm{q} 75$ & $\operatorname{Max}$ & Data References \\
\hline Forest & GPP & 74 & 387.00 & $1,064.0$ & $1,379.5$ & $1,786.0$ & $4,188.0$ & $\begin{array}{l}38,39,41,42,49,52,145,146,155,156,157,158,160,161,162,163,167,169,170,171,174,250, \\
257,283,288,289,545\end{array}$ \\
\hline Forest & $\mathrm{R}$ & 75 & 131.00 & 825.00 & $1,098.0$ & $1,333.2$ & $3,860.0$ & $\begin{array}{l}38,39,41,42,49,52,108,145,146,155,156,157,158,160,161,162,163,170,171,174,249,250, \\
257,283,288,289,545\end{array}$ \\
\hline Forest & NEP & 87 & -146.00 & 101.50 & 200.00 & 336.00 & 976.00 & $\begin{array}{l}38,39,41,42,49,145,146,151,155,156,157,158,160,161,162,163,165,166,168,170,171,174, \\
249,250,257,263,271,280,283,287,288,289,398,421,545\end{array}$ \\
\hline Forest & Detritus & 90 & 70.871 & 447.88 & $1,040.0$ & $2,491.9$ & 24,98 & $\begin{array}{l}36,37,38,40,41,42,63,64,73,148,149,150,151,153,154,164,173,183,224,226,248,258,259, \\
352,398,422,529,530\end{array}$ \\
\hline Forest & D. rate & 78 & 0.0120 & 0.2371 & 0.4334 & 0.9717 & 66.000 & $\begin{array}{l}91,95,122,135,152,176,184,201,224,225,226,228,229,248,249,251,252,253,255,256,354, \\
356,358\end{array}$ \\
\hline Grassland & GPP & 77 & 36.500 & 232.00 & 611.53 & $1,047.4$ & $3,723.0$ & $\begin{array}{l}47,54,81,86,87,88,92,100,167,177,178,188,189,190,257,278,279,283,285,286,394,400, \\
403,404,405,408,410,411,412,427,439,452\end{array}$ \\
\hline Grassland & $\mathrm{R}$ & 62 & 52.900 & 235.50 & 556.38 & $1,023.0$ & $5,725.6$ & $\begin{array}{l}35,47,54,70,71,72,76,81,86,87,88,92,100,177,178,189,257,260,278,279,283,285,286,394, \\
400,405,408,409,410,411,412,427,439,452,531\end{array}$ \\
\hline Grassland & NEP & 70 & -212.00 & -34.750 & 52.884 & 119.92 & $2,810.5$ & $\begin{array}{l}47,54,81,86,87,88,92,100,168,172,177,178,189,257,260,263,278,279,280,282,283,285 \\
286,377,394,397,400,405,408,410,411,412,427,439,452\end{array}$ \\
\hline Grassland & Detritus & 64 & 3.7568 & 88.198 & 176.81 & 279.35 & $2,250.0$ & $\begin{array}{l}44,46,51,54,75,85,150,151,175,180,187,282,377,378,399,406,410,413,414,415,424,426, \\
429,432,435,436,437,530,531\end{array}$ \\
\hline Grassland & D. flux & 5 & 10.585 & 12.447 & 13.104 & 13.322 & 20.988 & 376 \\
\hline Grassland & D. rate & 64 & 0.0120 & 0.1863 & 0.6230 & 1.0227 & 3.3731 & $\begin{array}{l}91,119,120,121,125,175,179,181,182,185,186,187,194,195,227,236,284,376,407,413,415, \\
423,425,426,531\end{array}$ \\
\hline Agroeco. & GPP & 24 & 599.00 & $1,286.8$ & $1,772.4$ & $1,992.6$ & $2,520.7$ & $167,232,257,277,279,281,402,428$ \\
\hline Agroeco. & $\mathrm{R}$ & 22 & 714.00 & $1,066.9$ & $1,309.5$ & $1,563.1$ & $1,996.0$ & $76,257,277,279,281,402,428$ \\
\hline Agroeco. & NEP & 21 & -115.00 & 155.00 & 303.00 & 361.05 & $1,204.5$ & $168,257,277,279,281,402,428$ \\
\hline Agroeco. & Detritus & 14 & 19.520 & 134.53 & 153.75 & 213.30 & 379.22 & $381,429,431,438,453$ \\
\hline Agroeco. & D. flux & 3 & 68.750 & 69.875 & 71.000 & 99.000 & 127.00 & 106 \\
\hline Agroeco. & D. rate & 12 & 0.5209 & 0.8503 & 1.1250 & 1.6598 & 2.6593 & $273,380,381,416,453,454$ \\
\hline Desert & GPP & 12 & 20.000 & 76.598 & 106.44 & 138.49 & 220.00 & $396,402,440,441,442,443,445,446$ \\
\hline Desert & $\mathrm{R}$ & 12 & 33.797 & 52.865 & 92.442 & 133.57 & 180.00 & $396,402,441,442,445,446,449,450$ \\
\hline Desert & NEP & 15 & -30.000 & 7.7626 & 32.596 & 62.483 & 130.41 & $151,280,391,395,396,402,441,442,444,445,446$ \\
\hline Desert & Detritus & 11 & 0.0000 & 0.9022 & 13.050 & 125.83 & 217.35 & $151,406,434,447,448,450$ \\
\hline Desert & D. rate & 12 & 0.0250 & 0.2672 & 0.5275 & 0.7105 & 1.4268 & $91,237,254,433,451$ \\
\hline Stream & GPP & 128 & 0.0000 & 13.470 & 55.240 & 155.36 & $1,770.0$ & $\begin{array}{l}12,15,18,28,89,90,93,94,96,97,110,111,138,159,203,207,209,210,214,218,234,235,239, \\
261,264,266,267,268,270,275,388,389,401,455,456,465,467,547,548\end{array}$ \\
\hline Stream & 2nd Prod. & 107 & 0.1132 & 2.1238 & 5.5056 & 14.175 & 287.75 & $\begin{array}{l}13,22,93,117,215,238,239,240,241,242,243,244,245,246,265,266,368,387,388,458,459, \\
460,472,476,477\end{array}$ \\
\hline Stream & $\mathrm{R}$ & 136 & 2.0323 & 76.093 & 290.41 & 617.58 & $2,148.9$ & $\begin{array}{l}12,15,28,89,93,96,110,111,138,159,203,207,208,210,214,218,234,235,245,261,264,266, \\
267,268,270,275,401,455,456,464,465,467,546,547,548,549\end{array}$ \\
\hline Stream & NEP & 129 & $-1,795.3$ & -400.46 & -114.10 & -20.900 & 568.95 & $\begin{array}{l}12,15,28,33,89,93,96,110,111,138,159,203,207,209,210,214,218,234,235,261,262,264, \\
267,268,270,271,274,275,276,401,455,456,465,467,547,548\end{array}$ \\
\hline Stream & Detritus & 139 & 0.0941 & 11.376 & 56.500 & 281.67 & $6,475.9$ & $\begin{array}{l}10,13,14,28,33,67,89,90,93,94,96,124,202,204,206,210,211,212,213,217,235,238,239, \\
240,242,244,266,321,388,392,417,457,466,470,471,474,475,540,541,542,543,544,549\end{array}$ \\
\hline Stream & D. rate & 100 & 0.1508 & 1.9109 & 4.0954 & 9.5022 & 203.90 & $\begin{array}{l}8,136,137,143,144,191,192,193,196,197,198,199,200,201,216,219,230,269,353,354,355, \\
357,359,360,361,362,363,366,389,392,468,469\end{array}$ \\
\hline Lake & GPP & 42 & 0.5270 & 16.796 & 53.050 & 161.67 & $1,922.0$ & $\begin{array}{l}20,53,79,101,112,114,115,290,292,293,295,297,316,317,318,323,336,379,487,488,489, \\
490,532,535,536\end{array}$ \\
\hline Lake & 2nd Prod. & 66 & 0.0200 & 1.6521 & 6.1314 & 19.443 & 442.00 & $246,247,290,291,295,332,336,368,473,482$ \\
\hline Lake & $\mathrm{R}$ & 44 & 10.092 & 17.653 & 37.999 & 85.757 & $1,100.5$ & $53,101,113,115,290,292,293,295,297,298,336,487,488,489,490,532,533,534,535,536$ \\
\hline Lake & NEP & 89 & -230.02 & -27.512 & -12.865 & -4.9348 & 65.922 & $\begin{array}{l}53,101,109,115,271,272,274,276,290,292,293,295,297,336,462,487,488,489,490,532,535, \\
536\end{array}$ \\
\hline Lake & Detritus & 43 & 0.7500 & 2.7875 & 4.7693 & 33.667 & $3,840.0$ & $53,68,298,307,320,322,323,336,379,461,463,485,486,491,537,538$ \\
\hline Lake & D. flux & 1 & 49.268 & 49.268 & 49.268 & 49.268 & 49.268 & 485 \\
\hline Lake & D. rate & 54 & 0.0167 & 0.2331 & 0.9424 & 1.9288 & 92.646 & $\begin{array}{l}68,105,107,139,140,141,142,231,272,316,323,376,379,478,479,480,481,483,484,486,492, \\
493\end{array}$ \\
\hline Oc. Pelagic & GPP & 66 & 0.7100 & 66.025 & 114.50 & 242.49 & 748.13 & $\begin{array}{l}55,80,102,116,126,128,129,130,131,132,133,304,305,309,310,311,312,313,314,315,319, \\
329,335,337,339,341,349,351,494,516\end{array}$ \\
\hline Oc. Pelagic & 2nd Prod. & 22 & 2.4360 & 7.1014 & 13.277 & 23.067 & 122.63 & $319,341,351,460,494,502,503,508,550,551,552,553,554,555$ \\
\hline Oc. Pelagic & $\mathrm{R}$ & 24 & 4.7433 & 55.635 & 112.36 & 217.95 & 521.28 & $304,305,313,314,315,319,329,335,337,339,341,344,349,351,494,516$ \\
\hline Oc. Pelagic & NEP & 71 & -69.927 & 17.507 & 40.000 & 86.868 & 447.00 & $43,45,48,116,304,305,313,314,315,319,329,330,337,339,341,348,349,351,494,507,516$ \\
\hline Oc. Pelagic & Detritus & 36 & 0.0180 & 2.0595 & 4.5426 & 12.660 & 334.49 & $129,130,311,315,319,328,334,337,338,339,344,348,349,351,457,494,513,514,516$ \\
\hline Oc. Pelagic & D. rate & 31 & 0.6492 & 2.1721 & 9.8550 & 44.632 & 788.40 & $126,127,132,133,319,327,328,376,495,515$ \\
\hline Oc. Benthic & GPP & 54 & 10.000 & 83.365 & 240.71 & 745.20 & $4,369.1$ & $82,83,84,221,222,223,299,300,301,311,313,315,324,333,339,340,343,344,349,350,506$ \\
\hline Oc. Benthic & 2nd Prod. & 42 & 0.3519 & 6.7409 & 11.400 & 25.715 & 745.90 & $311,331,350,494,501,502,503,504,506,507,508,510,511,512$ \\
\hline Oc. Benthic & $\mathrm{R}$ & 47 & 2.4717 & 84.390 & 253.43 & 570.15 & $2,737.5$ & $\begin{array}{l}83,221,222,223,233,299,300,301,304,305,311,313,315,319,324,331,333,339,340,344,349, \\
350\end{array}$ \\
\hline Oc. Benthic & NEP & 50 & -401.50 & -103.70 & -4.1724 & 39.542 & $2,120.7$ & $83,116,220,221,222,223,299,300,301,311,313,315,324,331,333,339,340,344,348,349,350$ \\
\hline
\end{tabular}




\begin{tabular}{|c|c|c|c|c|c|c|c|c|}
\hline Oc. Benthic & Detritus & 51 & 0.0250 & 8.5950 & 324.01 & 976.10 & $3,520.0$ & $300,302,303,311,319,325,326,339,347,348,350,498,501,502,503,504,505,507,508,509$ \\
\hline Oc. Benthic & D. flux & 2 & 15.600 & 47.700 & 79.800 & 111.90 & 144.00 & 342,393 \\
\hline Oc. Benthic & D. rate & 38 & 0.3855 & 1.2632 & 3.4537 & 6.9958 & 32.589 & $303,326,345,346,347,376,418,419,420,496,497,499$ \\
\hline
\end{tabular}

Oc. $=$ Ocean $; \mathrm{GPP}=$ Gross Primary Production; $\mathrm{R}=$ ecosystem respiration; $\mathrm{NEP}=\mathrm{Net}$ Ecosystem

Production; D. = Decomposition.

We provide the number of data points (n), the minimum, first quartile (q25), median, third quartile (q75) and maximum values of estimate distributions for each ecosystem $\mathrm{x}$ variable combination, as well as the associated references from which we extracted the data, listed in Supplementary Table 1. Values of stocks (Detritus), fluxes (GPP, R, NEP, $2^{\text {nd }}$ Prod, and D. flux) and rates (D. rates) are expressed in $\mathrm{gC} \mathrm{m}^{-2}, \mathrm{gC} \mathrm{m}^{-2}$ $\mathrm{yr}^{-1}$ and $\mathrm{yr}^{-1}$, respectively. 
Extended Data Table 4 I Factors used for conversions into grams of carbon.

\begin{tabular}{|c|c|c|c|c|c|c|c|c|c|}
\hline Type of material & KJ & Kcal & $\mathrm{molC}$ & $\mathrm{gCO}_{2}$ & $\mathrm{gO}_{2}$ & $\mathrm{molO}_{2}$ & g WW & g DW & g AFDW \\
\hline Organic Tissue ${ }^{\mathrm{a}}$ & 0.02 & 0.09 & 12 & 0.2727 & & & 0.09 & 0.45 & 0.5 \\
\hline Productivity, photosynthetic quotient $=1.2^{\mathrm{b}}$ & & & & & 0.3125 & 10 & & & \\
\hline Respiration, respiration quotient $=1^{\mathrm{ab}}$ & & & & & 0.375 & 12 & & & \\
\hline Non woody primary producer terrestrial ${ }^{\mathrm{c}}$ & & & & & & & & $1 / 3.33^{*}$ & \\
\hline Algae, sea grasses ${ }^{c}$ & & & & & & & $1 / 16.7$ & $1 / 2.92$ & \\
\hline Salamanders ${ }^{\mathrm{d}}$ & & & & & & & & 0.473 & \\
\hline Arthropods ${ }^{\mathrm{e}}$ & & & & & & & & 0.496 & \\
\hline
\end{tabular}

$\mathrm{KJ}=$ kilojoule; $\mathrm{Kcal}=$ kilocalorie molC $=$ mole of carbon; $\mathrm{gCO}_{2}=$ gram of carbon dioxide; $\mathrm{gO}_{2}=$ gram of di-oxygen; $\mathrm{molO}_{2}=$ mole of di-oxygen; $\mathrm{gWW}=$ gram of wet weight; $\mathrm{dDW}=$ gram of dry weight; $\mathrm{gAFDW}$ $=$ gram of ash-free dry weight.

${ }^{\text {a }}$ from Table 1 of Section II: Ecological Energetics, p26; in: Weathers, K. C., Strayer, D. L. \& Likens G. E. Fundamentals of ecosystem Science, (Academic Press, 2013); and references therein.

${ }^{\mathrm{b}}$ Irons III, J. G. and Oswood, M. W. Organic matter dynamics in 3 subarctic streams of interior Alaska, USA. J N Am Benthol Soc 16(1), 23-27 (1997); Duarte, C. M., et al. Seagrass community metabolism: Assessing the carbon sink capacity of seagrass meadows. Global Biogeochem. Cycles 24(4), 1-8 (2010); Huchette, S. M. H., Beveridge, M. C. M., Baird, D. J. \& Ireland, M. The impacts of grazing by tilapias Oreochromis niloticus L. on periphyton communities growing on artificial substrate in cages. Aquaculture 186, 45-60 (2000).

${ }^{\mathrm{c}}$ from Table 2.5 p26 In: Opitz S. Trophic Interactions in Caribbean Coral Reefs. Technical Reports 43, (International Center for Living Aquatic Resources Management, 1996).

${ }^{\mathrm{d}}$ Semlitsch, R. D., O’Donnell K. M. \& Thompson III, F.R. Abundance, biomass production, nutrient content, and the possible role of terrestrial salamanders in Missouri Ozark forest ecosystems. Can. J. Zool. 92, 997-1004 (2014).

${ }^{\text {e }}$ Small, G. E., Torres, P. J., Schweizer, L. M., Duff, J. H. \& Pringle, C. M. et al. Importance of terrestrial arthropods as subsidies in lowland Neotropical rain forest stream ecosystems. Biotropica 45(1), 80-87 (2013).

* used general conversion factor 0.45 in desert and arid grassland where the vegetation comprise woody species 
Extended Data Table 5 I Definition of flow drivers.

\begin{tabular}{|c|c|c|}
\hline & Definition & Example cross-ecosystem subsidy \\
\hline Passive drivers & & With reference to studies included in this study \\
\hline Fall / Wind & $\begin{array}{l}\text { Movement of terrestrial particles, detritus, or } \\
\text { organisms simply falling or windblown }\end{array}$ & $\begin{array}{l}\text { Tree leaves windblown in lakes }{ }^{53} \text {; terrestrial } \\
\text { arthropods falling in streams }{ }^{3} ; \text { mangrove arboricole } \\
\text { crabs falling in water and eaten by fish }\end{array}$ \\
\hline Leaching & $\begin{array}{l}\text { Movement of small particles or dissolved molecules } \\
\text { through diffusion in aquatic medium }\end{array}$ & Dissolved or particle organic carbon diffusing 430 \\
\hline Current / Tide & $\begin{array}{l}\text { Lateral movement of particles, detritus or } \\
\text { organisms triggered by water currents or tides }\end{array}$ & $\begin{array}{l}\text { Wrack grounding on beech } \\
\text { carcass deposited on beach }{ }^{104}\end{array}$ \\
\hline Sinking & $\begin{array}{l}\text { Vertical movements particles, detritus or organisms } \\
\text { triggered by gravity along the water column }\end{array}$ & 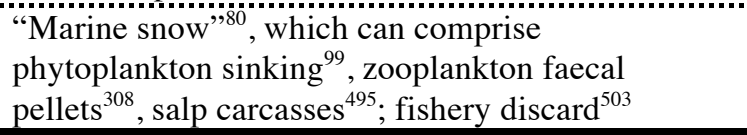 \\
\hline \multicolumn{3}{|l|}{ Active drivers } \\
\hline Foraging & $\begin{array}{l}\text { Daily movements of organisms to find food which } \\
\text { trigger cross-ecosystem movement of resources } \\
\text { (only those are considered here) }\end{array}$ & $\begin{array}{l}\text { Deer grazing in agro-ecosystems and defecating in } \\
\text { forests }{ }^{367} \text {; hippopotamus grazing in savannah and } \\
\text { defecating in river }{ }^{364} \text {; zooplankton eating in pelagic } \\
\text { area and finding refuge in deep sea }{ }^{384} \text {; bears } \\
\text { bringing salmon carcasses on land }{ }^{386}\end{array}$ \\
\hline Migration & $\begin{array}{l}\text { Seasonal long-distance movement of populations } \\
\text { triggering cross-ecosystem movement of resources }\end{array}$ & $\begin{array}{l}\text { Downing of wildebeest during seasonal } \\
\text { migration } 556\end{array}$ \\
\hline Life-cycle & $\begin{array}{l}\text { Cross-ecosystem organismal movements required } \\
\text { to complete a life-cycle }\end{array}$ & $\begin{array}{l}\text { Emergence of aquatic insects to land }{ }^{1} \text {; salamander } \\
\text { egg deposition in or emergence from aquatic } \\
\text { systems }^{60} \text {; anadromous salmon dying after breeding } \\
\text { in freshwater systems }\end{array}$ \\
\hline
\end{tabular}




\begin{tabular}{|c|c|c|c|c|c|c|c|c|}
\hline ID & Title & Authors & Year & Journal & Volume & Issue & Page & DOI / URL \\
\hline 1 & Ecosystem Linkages Between Lakes and the Surrounding Terrestrial Landscape in Northeast Iceland & Gratton C. et al. & 2008 & Ecosystems & 11 & 5 & $764-774$ & 10.1007/s10021-008-9158-8 \\
\hline 2 & Influence of streamside vegetation on inputs of terrestrial invertebrates to salmonid food webs & Allan J. D. et al. & 2003 & Can. J. of Fish. and Aq. Sci. & 60 & & $309-320$ & 10.1139/f03-019 \\
\hline 3 & $\begin{array}{l}\text { Terrestrial prey inputs to streams bordered by deciduous broadleaved forests, conifer plantations and clear- } \\
\text { cut sites in southwestern Japan: Effects on the abundance of red-spotted masu salmon }\end{array}$ & Inoue M. et al. & 2013 & Ecology of Freshwater Fish & 22 & 3 & $335-347$ & 10.1111/eff.12029 \\
\hline 4 & Arthropod 'rain' into tropical streams: The importance of intact riparian forest and influences on fish diets & Chan E. K. W. et al. & 2008 & Marine and Freshwater Research & 59 & & $653-660$ & 10.1071/MF07191 \\
\hline 5 & Fish invasion restructures stream and forest food webs by interrupting reciprocal prey subsidies & Baxter C. V. et al. & 2004 & Ecology & 85 & 10 & 2656-2663 & $10.1890 / 04-138$ \\
\hline 6 & $\begin{array}{l}\text { Invading rainbow trout usurp a terrestrial prey subsidy from native charr and reduce their growth and } \\
\text { abundance }\end{array}$ & Baxter C. V. et al. & 2007 & Oecologia & 153 & 2 & $461-470$ & $10.1007 / 500442-007-0743-x$ \\
\hline 7 & The energetic importance of terrestrial arthropod inputs to three warm-water streams & Cloe III, W. W. and Garman G. C. & 1996 & Freshwater Biology & 36 & 1 & $105-114$ & 10.1046/j.1365-2427.1996.00080.x \\
\hline 8 & $\begin{array}{l}\text { Effects of different types of conditioning on rates of leaf-litter shredding by Xiphocariselongata, a Neotropical } \\
\text { freshwater shrimp }\end{array}$ & Crowl T. A. et al. & 2006 & J. of the North Am. Benth. Soc. & 25 & 1 & $198-208$ & $\begin{array}{l}\text { 10.1899/0887- } \\
\text { 3593(2006)25[198:EODTOC]2.0.CO/2 }\end{array}$ \\
\hline 9 & Annual contribution of terrestrial invertebrates to a New Zealand trout stream & Edwards E. D. and Huryn A. D. & 1995 & $\begin{array}{l}\text { New Zealand Journal of Marine and } \\
\text { Freshwater Research }\end{array}$ & 29 & 4 & 467-477 & 10.1080/00288330.1995.9516680 \\
\hline 10 & $\begin{array}{l}\text { Linking stream habitats and spider distribution: spatial variations in trophic transfer across a forest-stream } \\
\text { boundary }\end{array}$ & Iwata T. & 2007 & Ecological Research & 22 & 4 & $619-628$ & 10.1007/s11284-006-0060-6 \\
\hline 11 & Stream meanders increase insectivorous bird abundance in riparian deciduous forests & Iwata T. et al. & 2003 & Ecography & 26 & & $325-337$ & 10.1034/j.1600-0587.2003.03355.x \\
\hline 12 & $\begin{array}{l}\text { Shifts in allochthonous input and autochthonous production in streams along an agricultural land-use } \\
\text { gradient }\end{array}$ & Hagen E. M. et al. & 2010 & Hydrobiolgia & 655 & 1 & $61-77$ & $0.1007 /$ s10750-010-0404-7 \\
\hline 13 & Effects of resource limitation on a detrital-based ecosystem & Wallace J. B. et al. & 1999 & Ecological Monographs & 69 & 4 & $409-442$ & $\begin{array}{l}\text { 10.1890/0012- } \\
\text { 9615(1999)069[0409:EORLOA]2.0.CO/2 }\end{array}$ \\
\hline 14 & Input and storage of benthic detritus in an Alaskan subarctic stream & Cowan C. A. and Oswood M. W. & 1983 & Polar Biology & 2 & & $35-40$ & 10.1007/BF00258283 \\
\hline 15 & Organic Matter Dynamics in 5 Subarctic Streams, Quebec, Canada & Naiman R. J. and Link L. G. & 1997 & J. of the North Am. Benth. Soc. & 16 & 1 & 33-39 & $10.2307 / 1468228$ \\
\hline 16 & $\begin{array}{l}\text { Hydrologic and Riparian Influences on the Import and Storage of Coarse Particulate Organic Matter in a } \\
\text { Prairie Stream }\end{array}$ & Gurtz M. E. et al. & 1988 & Can. J. of Fish. and Aq. Sci. & 45 & 4 & $655-665$ & 10.1139/f88-079 \\
\hline 17 & Production de litiere de la ripisylve de la Garonne et apport au fleuve & Chauvet E. and Jean-Louis A.-M. & 1988 & Acta Oecologica & 9 & 3 & $265-279$ & 10.1016/j.materresbull.2012.08.034 \\
\hline 18 & Carbon flow in a tundra stream ecosystem & Peterson B. J. et al. & 1986 & Can. J. of Fish. and Aq. Sci. & 43 & & 1259-1270 & 10.1139/f86-156 \\
\hline 19 & Prey use by web-building spiders: stable isotope analyses of trophic flow at a forest-stream ecotone & Kato C. et al. & 2004 & Ecological Research & 19 & 6 & $633-643$ & 10.1111/j.1440-1703.2004.00678.x \\
\hline 20 & Airborne litterfall as a source of organic matter in lakes & Gasith A. and Hasler A. D. & 1976 & Limnology and Oceanography & 21 & 2 & $253-258$ & 10.4319//o.1976.21.2.0253 \\
\hline 21 & Effects of Hemlock Mortality on Streams in the Southern Appalachian Mountains & Webster J. R. et al. & 2012 & The American Midland Naturalist & 168 & 1 & $112-131$ & 10.1674/0003-0031-168.1.112 \\
\hline 22 & Secondary Production, Emergence, and Export of Aquatic Insects of a Sonoran Desert Stream & Jackson J. K. and Fisher S. G. & 1986 & Ecology & 67 & 3 & $629-638$ & $10.2307 / 1937686$ \\
\hline 23 & Terrestrial invertebrate inputs determine the local abundance of stream fishes in a forested stream & Kawaguchi Y. et al. & 2003 & Ecology & 84 & 3 & $701-708$ & $\begin{array}{l}\text { 10.1890/0012- } \\
\text { 9658(2003)084[0701:TIIDTL]2.0.CO/2 }\end{array}$ \\
\hline 24 & Quantifying aquatic insect deposition from lake to land & Dreyer J. et al. & 2015 & Ecology & 96 & 2 & 499-509 & $10.1890 / 14-0704.1$ \\
\hline 25 & Importance of Terrestrial Arthropods as Subsidies in Lowland Neotropical Rain Forest Stream Ecosystems & Small G. E. et al. & 2013 & Biotropica & 45 & 1 & $80-87$ & 10.1111/j.1744-7429.2012.00896.x \\
\hline 26 & Organic Matter Dynamics in small streams of the Pacific Northwest & Richardson J. S. et al. & 2005 & $\begin{array}{l}\text { Journal of the American Water Resources } \\
\text { Association }\end{array}$ & 41 & 4 & 921-934 & 10.1111/j.1752-1688.2005.tb03777.x \\
\hline 27 & Riparian litter inputs to streams in the central Oregon Coast Range & Hart S. K. et al. & 2013 & Freshwater Science & 32 & 1 & 343-358 & 10.1899/12-074.1 \\
\hline 28 & An invasive riparian tree reduces stream ecosystem efficiency via a recalcitrant organic matter subsidy & Mineau M. M. et al. & 2012 & Ecology & 93 & 7 & 1501-1508 & 10.1890/11-1700.1 \\
\hline 29 & $\begin{array}{l}\text { Regulation leads to increases in riparian vegetation, but not direct allochthonous inputs, along the Colorado } \\
\text { River in Grand Canyon, Arizona }\end{array}$ & Kennedy T. A. and Ralston B. E. & 2012 & River Research and Applications & 28 & & & 10.1002/rra.1431 \\
\hline 30 & Particulate Allochthonous Inputs: Relationships with Stream Size in an Undisturbed Watershed & Conners M. E. \& Naiman R. J. & 1984 & Can. J. of Fish. and Aq. Sci. & 41 & & 1473-1484 & 10.1139/f84-181 \\
\hline 31 & Litterfall, litter decomposition, and flux of particulate organic material in a coastal plain stream & Post H. A. and De La Cruz A. A. & 1977 & Hydrobiolgia & 55 & & $201-207$ & $10.1007 / \mathrm{BF} 00017551$ \\
\hline 32 & $\begin{array}{l}\text { Contribution of terrestrial invertebrates to the annual resource budget for salmonids in forest and grassland } \\
\text { reaches of a headwater stream }\end{array}$ & Kawagushi Y. and Nakano $\mathrm{S}$. & 2001 & Freshwater Biology & 46 & & 303-316 & 10.1046/j.1365-2427.2001.00667.x \\
\hline
\end{tabular}




\begin{tabular}{|c|c|c|c|c|c|c|c|c|}
\hline 33 & Energy Flow in Bear Brook, New Hampshire: An Integrative Approach to Stream Ecosystem Metabolism & Fisher S. G. \& Likens G. E. & 1973 & Ecological Monographs & 43 & 4 & 421-439 & $10.2307 / 1942301$ \\
\hline 34 & $\begin{array}{l}\text { Temporal variability in insectivorous bat activity along two desert streams with contrasting patterns of prey } \\
\text { availability }\end{array}$ & Hagen M. H. and Sabo J. L. & 2014 & Journal of Arid Environments & 102 & & 104-112 & 10.1016/j.jaridenv.2013.11.016 \\
\hline 35 & The importance of winter in annual ecosystem respiration in the High Arctic & Morgner E. et al. & 2010 & Polar Research & 29 & 1 & $58-74$ & 10.1111/j.1751-8369.2010.00151.x \\
\hline 36 & Boreal forest and Tundra. & Apps M. J. et al & 1993 & $\begin{array}{l}\text { In: Terrestrial Biospheric Carbon Fluxes: } \\
\text { Quantification of Sinks and Sources of } \mathrm{C} 02 .\end{array}$ & & & $39-54$ & 10.1007/978-94-011-1982-5 \\
\hline 37 & Studies on the Biomass and Productivity in a Series of Montane Rain Forests in Jamaica & Tanner E. V.J. & 1980 & Journal of Ecology & 68 & 2 & $573-588$ & $10.2307 / 2259423$ \\
\hline 38 & $\begin{array}{l}\text { Integrating aquatic and terrestrial components to construct a complete carbon budget for a north temperate } \\
\text { lake district }\end{array}$ & Buffam I. et al. & 2011 & Global Change Biology & 17 & 2 & 1193-1211 & 10.1111/j.1365-2486.2010.02313.x \\
\hline 39 & Climatic controls on the carbon and water balances of a boreal aspen forest, 1994-2003 & Barr A. G. et al. & 2007 & Global Change Biology & 13 & 3 & $561-576$ & 10.1111/j.1365-2486.2006.01220.x \\
\hline 40 & $\begin{array}{l}\text { Implications of increased deciduous cover on stand structure and aboveground carbon pools of Alaskan } \\
\text { boreal forests }\end{array}$ & Alexander $\mathrm{H}$. et al. & 2012 & Ecosphere & 3 & 5 & Art.45 & 10.1890/ES11-00364.1 \\
\hline 41 & Production, Respiration, and Overall Carbon Balance in an Old-growth Pseudotsuga-Tsuga Forest Ecosystem & Harmon M. E. et al. & 2004 & Ecosystems & 7 & & 498-512 & 10.1007/s10021-004-0140-9 \\
\hline 42 & Carbon balance of different aged Scots pine forests in Southern Finland & Kolari P. et al. & 2004 & Global Change Biology & 10 & 7 & 1106-1119 & 10.1111/j.1365-2486.2004.00797.x \\
\hline 43 & $\begin{array}{l}\text { Synthesis of primary production in the Arctic Ocean: III. Nitrate and phosphate based estimates of net } \\
\text { community production }\end{array}$ & Codispoti L. A. et al. & 2013 & Progress in Oceanography & 110 & & $126-150$ & 10.1016/j.pocean.2012.11.006 \\
\hline 44 & Temporal variability in plant and soil nitrogen pools in a high-Arctic ecosystem & Bardgett R. D. et al. & 2007 & Soil Biology and Biochemistry & 39 & 8 & 2129-2137 & 10.1016/j.soilbio.2007.03.016 \\
\hline 45 & Annual net community production and the biological carbon flux in the ocean & Emerson $\mathrm{S}$. & 2014 & Global Biogeochemical Cycles & 28 & & $14-28$ & $10.1002 / 2013 G B 004680$ \\
\hline 46 & Primary Production in Arctic Cottongrass Tussock Tundra Communities & Wein R. W. and Bliss L. C. & 1974 & Arctic and Alpine Research & 6 & 3 & 261-274 & $10.2307 / 1550062$ \\
\hline 47 & CO2 exchange in three Canadian High Arctic ecosystems: Response to long-term experimental warming & Welker J. M. et al. & 2004 & Global Change Biology & 10 & 12 & 1981-1995 & 10.1111/j.1365-2486.2004.00857.x \\
\hline 48 & $\begin{array}{l}\text { Global net community production estimated from the annual cycle of surface water total dissolved inorganic } \\
\text { carbon }\end{array}$ & Lee $\mathrm{K}$. & 2001 & Limnology and Oceanography & 46 & 6 & 1287-1297 & 10.4319/lo.2001.46.6.1287 \\
\hline 49 & $\begin{array}{l}\text { Influence of temperature and drought on seasonal and interannual variations of soil, bole and ecosystem } \\
\text { respiration in a boreal aspen stand }\end{array}$ & Gaumont-Guay D. et al. & 2006 & Agricultural and Forest Meteorology & 140 & & 203-219 & 10.1016/j.agrformet.2006.08.002 \\
\hline 50 & $\begin{array}{l}\text { Blowing litter across a landscape: effects on ecosystem nutrient flux and implications for landscape } \\
\text { management }\end{array}$ & Shen W. et al. & 2011 & Landscape Ecology & 26 & 5 & 629-644 & 10.1007/s10980-011-9599-x \\
\hline 51 & Impacts of Grazing and Climate Warming on C Pools and Decomposition Rates in Arctic Environments & Sjögersten S. et al. & 2012 & Ecosystems & 15 & 3 & 349-362 & 10.1007/s10021-011-9514-y \\
\hline 52 & Primary production and carbon allocation in relation to nutrient supply in a tropical experimental forest & Giardina C. P. et al. & 2003 & Global Change Biology & 9 & & 1438-1450 & 10.1046/j.1365-2486.2003.00558.x \\
\hline 53 & $\begin{array}{l}\text { A Carbon Budget of a Small Humic Lake: An Example of the Importance of Lakes for Organic Matter Cycling in } \\
\text { Boreal Catchments }\end{array}$ & Sobek S. et al. & 2006 & AMBIO: A Journal of the Human Environment & 35 & 8 & $469-475$ & $\begin{array}{l}\text { 10.1579/0044- } \\
7447(2006) 35[469: A C B O A S] 2.0 . C O / 2\end{array}$ \\
\hline 54 & Carbon balance of a tropical savanna of northern Australia & Chen X. et al. & 2003 & Oecologia & 137 & 3 & $405-416$ & $10.1007 / 500442-003-1358-5$ \\
\hline 55 & Phytoplankton phenology and production around Iceland and Faroes & Zhai L. et al. & 2012 & Continental Shelf Research & 37 & & $15-25$ & 10.1016/j.csr.2012.01.013 \\
\hline 56 & Redistributed lacustrine detritus as a spatial subsidy of biological resources for soils in an Antarctic dry valley & Hopkins D. W. et al. & 2008 & Geoderma & 144 & $1-2$ & 86-92 & 10.1016/j.geoderma.2007.10.016 \\
\hline 57 & Hydrologic Influences on Insect Emergence Production from Central Platte River Wetlands & Whiles M. R. and Goldowitz B. S. & 2001 & Ecological Applications & 11 & 6 & $1829-1842$ & $\begin{array}{l}\text { 10.1890/1051- } \\
0761(2001) 011[1829: \mathrm{HIOIEP}] 2.0 . \mathrm{CO} / 2\end{array}$ \\
\hline 58 & $\begin{array}{l}\text { Variation in the trophic basis of production and energy flow associated with emergence of larval salamander } \\
\text { assemblages from forest ponds }\end{array}$ & Regester K. J. et al. & 2008 & Freshwater Biology & 53 & 9 & 1754-1767 & 10.1111/j.1365-2427.2008.02073.x \\
\hline 59 & $\begin{array}{l}\text { Remarkable Amphibian Biomass and Abundance in an Isolated Wetland: Implications for Wetland } \\
\text { Conservation }\end{array}$ & Gibbons J. W. et al. & 2006 & Conservation Biology & 20 & 5 & 1457-1465 & 10.1111/j.1523-1739.2006.00443.x \\
\hline 60 & $\begin{array}{l}\text { Linking the evolution of habitat choice to ecosystem functioning: direct and indirect effects of pond- } \\
\text { reproducing fire salamanders on aquatic-terrestrial subsidies. }\end{array}$ & Reinhardt T. et al. & 2013 & Oecologia & 173 & 1 & 281-291 & 10.1007/s00442-013-2592-0 \\
\hline 61 & $\begin{array}{l}\text { Decomposition Rates of Salamander (Ambystoma Maculatum) Life Stages and Associated Energy and } \\
\text { Nutrient Fluxes in Ponds and Adjacent Forest in Southern Illinois }\end{array}$ & Regester K. J. and Whiles M. R. & 2006 & Copeia & 2006 & 4 & 640-649 & $\begin{array}{l}\text { 10.1643/0045- } \\
\text { 8511(2006)6[640:DROSAM]2.0.CO/ }\end{array}$ \\
\hline 62 & $\begin{array}{l}\text { Emergence of Aquatic Insects from } 2 \text { Habitats in a Small Wetland of the Southeastern USA: Temporal Patterns } \\
\text { of Numbers and Biomass }\end{array}$ & Stagliano D. M. et al. & 1998 & J. of the North Am. Benth. Soc. & 17 & 1 & $37-53$ & $10.2307 / 1468050$ \\
\hline 63 & Rates of Forest Floor and Nutrient Turnover in Aspen, Pine, and Spruce Stands on Two Different Soils & Perala D. A. and Alban D. H. & 1982 & Res. Pap. NC-227. St Paul, MN: U.S. Dep. of Agri & culture, & Eorest & Service, Nort & rth Central Forest Experiment Station. $5 \mathrm{p}$ \\
\hline 64 & A Comparative Analysis of Potential Nitrification and Nitrate Mobility in Forest Ecosystems & Vitousek P. M. et al. & 1982 & Ecological Monographs & 52 & 2 & 155-157 & $10.2307 / 1942609$ \\
\hline 65 & Emergence production and export of aquatic insects from a tallgrass prairie stream & Gray L.J. & 1989 & The Southwestern Naturalist & 34 & 3 & $313-318$ & $10.2307 / 3672158$ \\
\hline 66 & Life history and production of a semi-terrestrial limnephilid caddisfly in an intermittent Platte River wetland & Whiles M. R. et al. & 1999 & J. of the North Am. Benth. Soc. & 18 & 4 & 533-544 & $10.2307 / 1468385$ \\
\hline 67 & Longitudinal patterns of ecosystem processes and community structure in a subartic river continuum & Naiman R. J. et al. & 1987 & Ecology & 68 & 5 & 1139-1156 & 10.2307/1939199 \\
\hline
\end{tabular}




\begin{tabular}{|c|c|c|c|c|c|c|c|c|}
\hline 68 & Major findings (book section) & Hobbie J. E. et al. & 1980 & $\begin{array}{l}\text { In: Limnology of tundra ponds, Barrow, } \\
\text { Alaska }\end{array}$ & & & $1-19$ & \\
\hline 69 & Benthic secondary production and biomass of insects emerging from a northern German temperate stream & Poepperl R. & 2000 & Freshwater Biology & 44 & 2 & 199-211 & 10.1046/j.1365-2427.2000.00558.x \\
\hline 70 & Soil carbon dioxide fluxes in northern semiarid grasslands & Frank A. B. et al. & 2002 & Soil Biology and Biochemisty & 34 & 9 & 1235-1241 & $10.1016 / 50038-0717(02) 00062-7$ \\
\hline 71 & Responses of Soil Respiration to Clipping and Grazing in a Tallgrass Prairie & Bremer D. J. et al. & 1998 & Journal of Environmental Quality & 27 & 6 & $1539-1548$ & $\begin{array}{l}10.2134 / \text { jeq1998.00472425002700060034 } \\
x\end{array}$ \\
\hline 72 & Soil $\mathrm{CO} 2$ flux in a tallgrass prairie & Mielnick P. C. and Dugas W. A. & 2000 & Soil Biology and Biochemisty & 32 & 2 & $221-228$ & $10.1016 / 50038-0717(99) 00150-9$ \\
\hline 73 & A 70-year retrospective analysis of carbon fluxes in the Canadian Forest Sector & Kurz W. A. and Apps M. J. & 1999 & Ecological Applications & 9 & 2 & $526-547$ & $\begin{array}{l}\text { 10.1890/1051- } \\
\text { 0761(1999)009[0526:AYRAOC]2.0.CO/2 }\end{array}$ \\
\hline 74 & Dynamics of aquatic insect flux affects distribution of riparian web-building spiders & Kato C. et al. & 2003 & Oikos & 103 & $\begin{array}{c}\text { Marc } \\
h\end{array}$ & $113-120$ & 10.1034/j.1600-0706.2003.12477.x \\
\hline 75 & Productivity and carbon fluxes of tropical savannas & Grace J. et al. & 2006 & Journal of Biogeography & 33 & & $387-400$ & 10.1111/j.1365-2699.2005.01448.x \\
\hline 76 & Tillage-induced soil carbon dioxide loss from different cropping systems & Reicosky D. C. et al. & 1997 & Soil and Tillage Research & 41 & $1-2$ & $105-118$ & $10.1016 / 50167-1987(96) 01080-X$ \\
\hline 77 & Aquatic prey subsidies to riparian spiders in a stream with different land use types & Krell B. et al. & 2015 & Limnologica & 51 & & $1-7$ & 10.1016/j.limno.2014.10.001 \\
\hline 78 & Land-use effects on terrestrial consumers through changed size structure of aquatic insects & Stenroth K. et al. & 2015 & Freshwater Biology & 60 & 1 & $136-149$ & 10.1111/fwb.12476 \\
\hline 79 & $\begin{array}{l}\text { Whole-lake estimates of carbon flux through algae and bacteria in benthic and pelagic habitats of clear-water } \\
\text { lakes }\end{array}$ & Ask J. et al. & 2009 & Ecology & 90 & 7 & 1923-1932 & 10.1890/07-1855.1 \\
\hline 80 & Revisiting Carbon Flux Through the Ocean's Twilight Zone & Buesseler K. O. et al. & 2007 & Science & 316 & April & $567-570$ & 10.1126/science.1137959 \\
\hline 81 & Permafrost degradation stimulates carbon loss from experimentally warmed tundra & Natali S. M. et al. & 2014 & Ecology & 95 & 3 & $602-608$ & $10.1890 / 13-0602.1$ \\
\hline 82 & Patterns of primary production and nutrient availability in a Bahamas lagoon with fringing mangroves & Koch M. S. and Madden C. J. & 2001 & Marine Ecology Progress Series & 219 & & $109-119$ & 10.3354/meps219109 \\
\hline 83 & Mass balance constraints on nutrient cycling in tropical seagrass beds & $\begin{array}{l}\text { Erftemeijer P. L. A. and } \\
\text { Middelburg J. J. }\end{array}$ & 1995 & Aquatic Botany & 50 & & 21-36 & 10.1016/0304-3770(94)00440-W \\
\hline 84 & $\begin{array}{l}\text { Production and consumption of tropical seagrass fields in Eastern Indonesia measured with bell jars and } \\
\text { microelectrodes }\end{array}$ & $\begin{array}{l}\text { Lindeboom H. J. and Sandee A. } \\
\text { J. J. }\end{array}$ & 1989 & Netherlands Journal of Sea Research & 23 & 2 & $181-190$ & 10.1016/0077-7579(89)90012-4 \\
\hline 85 & $\begin{array}{l}\text { Biogeochemical pools and fluxes of carbon and nitrogen in a maritime tundra near penguin colonies along the } \\
\text { Antarctic Peninsula }\end{array}$ & Park J.-H. et al. & 2007 & Polar Biology & 30 & 2 & 199-207 & DOI 10.1007/s00300-006-0173-y \\
\hline 86 & $\begin{array}{l}\text { Seasonal patterns of carbon dioxide and water fluxes in three representative tundra ecosystems in northern } \\
\text { Alaska }\end{array}$ & Euskirchen E. S. et al. & 2012 & Ecosphere & 3 & 1 & Art.4 & 10.1890/ES11-00202.1 \\
\hline 87 & Longer growing seasons do not increase net carbon uptake in the northeastern Siberian tundra & Parmentier F. J. W. et al. & 2011 & $\begin{array}{l}\text { Journal of Geophysical Research: } \\
\text { Biogeosciences }\end{array}$ & 116 & 4 & G04013 & 10.1029/2011JG001653 \\
\hline 88 & Trends in CO2 exchange in a high Arctic tundra heath, 2000-2010 & Lund M. et al. & 2012 & $\begin{array}{l}\text { Journal of Geophysical Research: } \\
\text { Biogeosciences }\end{array}$ & 117 & & G02001 & 10.1029/2011JG001901 \\
\hline 89 & Organic matter dynamics in the Kuparuk River, a tundra river in Alaska, USA & Harvey C. J. et al. & 1997 & J. of the North Am. Benth. Soc. & 16 & 1 & $18-23$ & $10.2307 / 1468225$ \\
\hline 90 & Organic matter dynamics in 3 subarctic streams of interior Alaska, USA & Irons III J. G. and Oswood M. W. & 1997 & J. of the North Am. Benth. Soc. & 16 & 1 & 23-27 & $10.2307 / 1468226$ \\
\hline 91 & $\begin{array}{l}\text { Net primary and ecosystem production and carbon stocks of terrestrial ecosystems and their responses to } \\
\text { climate change }\end{array}$ & Cao M. and Woodward I. F. & 1998 & Global Change Biology & 4 & 2 & 185-198 & 10.1046/j.1365-2486.1998.00125.x \\
\hline 92 & The growing season greenhouse gas balance of a continental tundra site in the Indigirka lowlands, NE Siberia & Van der Molen M. K. et al. & 2007 & Biogeosciences & 4 & 6 & $985-1003$ & $10.5194 / \mathrm{bg}-4-985-2007$ \\
\hline 93 & Organic Matter Processing by a Stream-Segment Ecosystem: Fort River, Massachusetts, U.S.A. & Fisher S. G. & 1977 & $\begin{array}{l}\text { Internationale Revue der gesamten } \\
\text { Hydrobiologie und Hydrographie }\end{array}$ & 62 & 6 & 701-727 & 10.1002/iroh.1977.3510620601 \\
\hline 94 & Organic Matter Dynamics in Sycamore Creek, a Desert Stream in Arizona, USA & Jones J. B. et al. & 1997 & J. of the North Am. Benth. Soc. & 16 & 1 & $78-82$ & $10.2307 / 1468238$ \\
\hline 95 & Belowground biomass, production, and carbon cycling in mature Norway spruce, Maine, U.S.A. & Cronan C.S. & 2003 & Canadian Journal of Forest Research & 33 & 2 & $339-350$ & $10.1139 / \times 02-189$ \\
\hline 96 & Organic Matter Dynamics in the West Fork of Walker Branch, Tennessee, USA & Mulholland P. J. & 1997 & J. of the North Am. Benth. Soc. & 16 & 1 & $61-67$ & $10.2307 / 1468235$ \\
\hline 97 & Canada Stream: a glacial meltwater stream in Taylor Valley, South Victoria Land, Antarctica & Mcknight D. M. and Tate C. M. & 1997 & J. of the North Am. Benth. Soc. & 16 & 1 & $14-17$ & $10.2307 / 1468224$ \\
\hline 98 & The input of terrestrial invertebrates from tree canopies to a stream & $\begin{array}{l}\text { Mason C. F. and MacDonald S. } \\
\text { M. }\end{array}$ & 1982 & Freshwater Biology & 12 & 4 & 305-311 & 10.1111/j.1365-2427.1982.tb00624.x \\
\hline 99 & An algal carbon budget for pelagic-benthic coupling in Lake Michigan & $\begin{array}{l}\text { Fitzgerald S. A. and Gardner W. } \\
\text { S. }\end{array}$ & 1993 & Limnology and Oceanography & 38 & 3 & $547-560$ & 10.4319/lo.1993.38.3.0547 \\
\hline 100 & Carbon balance in East European tundra & Heikkinen J. E. P. et al. & 2004 & Global Biogeochemical Cycles & 18 & 1 & GB1023 & 10.1029/2003GB002054 \\
\hline 101 & Benthic Photosynthesis and Respiration in Char Lake & Welch H. E. and Kalff J. & 1974 & $\begin{array}{l}\text { Journal of the Fisheries Research Board of } \\
\text { Canada }\end{array}$ & 31 & 5 & $609-620$ & 10.1139/f74-093 \\
\hline 102 & Annual primary production and export flux in the Southern Ocean from sediment trap data & Wefer $\mathrm{G}$. and Fischer $\mathrm{G}$. & 1991 & Marine Chemistry & 35 & $1-4$ & $597-613$ & $10.1016 / 50304-4203(09) 90045-7$ \\
\hline 103 & $\begin{array}{l}\text { Extraordinarily high spider densities on islands: flow of energy from the marine to terrestrial food webs and } \\
\text { the absence of predation }\end{array}$ & Polis G. A. and Hurd S. D. & 1995 & $\begin{array}{l}\text { Proceedings of the National Academy of } \\
\text { Sciences }\end{array}$ & 92 & 10 & 4382-4386 & 10.1073/pnas.92.10.4382 \\
\hline
\end{tabular}




\begin{tabular}{|c|c|c|c|c|c|c|c|c|}
\hline 104 & $\begin{array}{l}\text { Linking Marine and Terrestrial Food Webs: Allochthonous Input from the Ocean Supports High Secondary } \\
\text { Productivity on Small Islands and Coastal Land Communities }\end{array}$ & Polis G. A. and Hurd S. D. & 1996 & The American Naturalist & 147 & 3 & $396-423$ & http://www.jstor.org/stable/2463215 \\
\hline 105 & Regeneration of Nutrient and Detritus Formation from Aerobic Decomposition of Natural Phytoplankton & Aizaki M. and Takamura N. & 1991 & Japanese Journal of Limnology & 52 & 2 & $83-94$ & 10.3739/rikusui.52.83 \\
\hline 106 & Organic carbon and nitrogen flows & Andrén O. et al. & 1990 & Ecological bulletins & 40 & May & $85-126$ & http://www.jstor.org/stable/20113040 \\
\hline 107 & $\begin{array}{l}\text { Differentiating the degradation dynamics of algal and terrestrial carbon within complex natural dissolved } \\
\text { organic carbon in temperate lakes }\end{array}$ & Guillemette F. et al. & 2013 & $\begin{array}{l}\text { Journal of Geophysical Research: } \\
\text { Biogeosciences }\end{array}$ & 118 & & 963-973 & 10.1002/jgrg.20077 \\
\hline 108 & $\begin{array}{l}\text { Ecosystem respiration in two Mediterranean evergreen Holm Oak forests: drought effects and decomposition } \\
\text { dynamics }\end{array}$ & Reichstein M. et al. & 2002 & Functional ecology & 16 & & $27-39$ & 10.1046/j.0269-8463.2001.00597.x \\
\hline 109 & Drivers of metabolism and net heterotrophy in contrasting lakes & Staehr P. A. et al. & 2010 & Limnology and Oceanography & 55 & 2 & $817-830$ & 10.4319/lo.2010.55.2.0817 \\
\hline 110 & $\begin{array}{l}\text { Multiple Scales of Temporal Variability in Ecosystem Metabolism Rates: Results from } 2 \text { Years of Continuous } \\
\text { Monitoring in a Forested Headwater Stream }\end{array}$ & Roberts B. J. et al. & 2007 & Ecosystems & 10 & 4 & $588-606$ & 10.1007/s10021-007-9059-2 \\
\hline 111 & $\begin{array}{l}\text { Annual cycle and inter-annual variability of gross primary production and ecosystem respiration in a } \\
\text { floodprone river during a } 15 \text {-year period }\end{array}$ & Uehlinger $U$. & 2006 & Freshwater Biology & 51 & 5 & $938-950$ & 10.1111/j.1365-2427.2006.01551.x \\
\hline 112 & Nutrient Supply and Prirnary Production in Clear Lake, Eastern Ontario & $\begin{array}{l}\text { Schindler D. W. and } \\
\text { Nighswander J. E. }\end{array}$ & 1970 & $\begin{array}{l}\text { Journal of the Fisheries Research Board of } \\
\text { Canada }\end{array}$ & 27 & 11 & 2009-2036 & 5 10.1139/f70-226 \\
\hline 113 & Metabolic rates of Arctic lakes & Welch H. E. & 1974 & Limnology and Oceanography & 19 & 1 & $65-73$ & 10.4319/lo.1974.19.1.0065 \\
\hline 114 & Epipelic and pelagic primary production in Alaskan Arctic lakes of varying depth & Whalen S. C. et al. & 2008 & Hydrobiologia & 614 & 1 & $243-257$ & $0.1007 / \mathrm{s} 10750-008-9510-1$ \\
\hline 115 & The Ecosystem of Thingvallavatn: A Synthesis & Jonasson P. M. & 1992 & Oikos & 64 & $1-2$ & $405-434$ & $10.2307 / 3545062$ \\
\hline 116 & $\begin{array}{l}\text { Intra-regional comparison of productivity, carbon flux and ecosystem composition within the northern } \\
\text { Barents Sea }\end{array}$ & Reigstad M. et al. & 2011 & Progress in Oceanography & 90 & $1-4$ & $33-46$ & 10.1016/j.pocean.2011.02.005 \\
\hline 117 & Relative importance of carbon sources for macroinvertebrates in a Rocky Mountain stream & $\begin{array}{l}\text { McCutchan J. H. Jr. and Lewis } \\
\text { W. M. Jr. }\end{array}$ & 2002 & Limnology and Oceanography & 47 & 3 & $742-752$ & 10.4319/lo.2002.47.3.0742 \\
\hline 118 & Particulate organic matter inputs to a glacial stream ecosystem in the Swiss Alps & Zah R. and Uehlinger U. & 2001 & Freshwater Biology & 46 & 12 & $1597-1608$ & B 10.1046/j.1365-2427.2001.00847.x \\
\hline 119 & Structure and Function of Tundra Ecosystems: Devon Island, Canada & Bliss L.C. & 1975 & Ecological Bulletin & 20 & & $17-60$ & \\
\hline 120 & Structure and Function of Tundra Ecosystems:Barrow, Alaska, USA & Bunnell FL et al. & 1975 & Ecological Bulletin & 20 & & $73-124$ & \\
\hline 121 & Structure and Function of Tundra Ecosystems:Agapa, USSR & Vassiljevskaya V. D. et al. & 1975 & Ecological Bulletin & 20 & & $141-158$ & \\
\hline 122 & Structure and Function of Tundra Ecosystems: Kevo, Finland & Kallio P. & 1975 & Ecological Bulletin & 20 & & $193-224$ & \\
\hline 123 & Litter inputs to a tropical Australian rainforest stream & Benson L. J. and Pearson R. G. & 1993 & Australian Journal of Ecology & 18 & 4 & $377-383$ & 10.1111/j.1442-9993.1993.tb00465.x \\
\hline 124 & $\begin{array}{l}\text { Allochthonous litter inputs, organic matter standing stocks, and organic seston dynamics in upland } \\
\text { Panamanian streams: potential effects of larval amphibians on organic matter dynamics }\end{array}$ & Colon-Gaud C. et al. & 2008 & Hydrobiologia & 603 & 1 & 301-312 & 10.1007/s10750-008-9294-3 \\
\hline 125 & $\begin{array}{l}\text { Plant litter decomposition in a semi-arid ecosystem controlled by photodegradation. } \\
\end{array}$ & Austin A. T. and Vivanco L. & 2006 & Nature & 442 & 7102 & $555-558$ & $10.1038 /$ nature05038 \\
\hline 126 & Biomass and production of heterotrophic bacterioplankton in the oceanic subarctic Pacific & Kirchman D. L. et al. & 1993 & $\begin{array}{l}\text { Deep Sea Research Part I: Oceanographic } \\
\text { Research Papers }\end{array}$ & 40 & 5 & 967-988 & 10.1016/0967-0637(93)90084-G \\
\hline 127 & Major role of bacteria in biogeochemical fluxes in the ocean's interior & Cho B.C. and Azam F. & 1988 & Nature & 332 & 6163 & $441-443$ & $10.1038 / 332441 \mathrm{a} 2$ \\
\hline 128 & Primary production in the North Pacific Central Gyre: some new measurements based on $14 \mathrm{C}$ & Marra J. and Heinemann K. R. & 1987 & $\begin{array}{l}\text { Deep Sea Research Part A. Oceanographic } \\
\text { Research Papers }\end{array}$ & 34 & 11 & $1821-1829$ & 10.1016/0198-0149(87)90056-2 \\
\hline 129 & Role of plankton in the carbon and nitrogen budgets of Santa Monica Basin, California & Small L. F. et al. & 1989 & Marine ecology Progress series & 56 & & $57-74$ & $10.3354 /$ meps056057 \\
\hline 130 & $\begin{array}{l}\text { Organic carbon partitioning during spring phytoplankton blooms in the Ross Sea polynya and the Sargasso } \\
\text { Sea }\end{array}$ & Carlson C. A. et al. & 1998 & Limnology and Oceanography & 43 & 3 & $375-386$ & 10.4319/lo.1998.43.3.0375 \\
\hline 131 & $\begin{array}{l}\text { Overview of the US JGOFS Bermuda Atlantic Time-series Study (BATS): A decade-scale look at ocean biology } \\
\text { and biogeochemistry }\end{array}$ & Steinberg D. K. et al. & 2001 & $\begin{array}{l}\text { Deep-Sea Research Part II: Topical Studies in } \\
\text { Oceanography }\end{array}$ & 48 & $8-9$ & $1405-1447$ & 10.1016/s0967-0645(00)00148-X \\
\hline 132 & Minireview: The bacterial content of the oceanic euphotic zone & Ducklow H.W. & 1999 & FEMS Microbiology-Ecology & 30 & 1 & $1-10$ & 10.1111/j.1574-6941.1999.tb00630.x \\
\hline 133 & Benthic-pelagic coupling in the Greenland-Norwegian Sea and its effect on the geological record & Graf G. et al. & 1995 & Geologische Rundschau & 84 & 1 & 49-58 & 10.1007/BF00192241 \\
\hline 134 & $\begin{array}{l}\text { Pelagic-benthic coupling: Profundal benthic community response to spring diatom deposition in mesotrophic } \\
\text { Lake Erken }\end{array}$ & Goedkoop W. and Johnson R. K. & 1996 & Limnology and Oceanography & 41 & 4 & $636-647$ & 10.4319/lo.1996.41.4.0636 \\
\hline 135 & Decomposition of ponderosa pine needles in a Mediterranean-type climate & Hart S. C. et al. & 1992 & Canadian Journal of Forest Research & 22 & 3 & $306-314$ & $10.1139 / \times 92-040$ \\
\hline 136 & Stable successional patterns of aquatic hyphomycetes on leaves decaying in a summer cool stream & Gessner M. O. et al & 1993 & Mycological Research & 97 & 2 & $163-172$ & $10.1016 / 50953-7562(09) 80238-4$ \\
\hline 137 & The role of leaf litter macroinvertebrates in stream-floodplain dynamics & Merrit R. W. and Lawson D. L. & 1992 & Hydrobiologia & 248 & 1 & $65-77$ & $10.1007 / \mathrm{BF} 00008886$ \\
\hline 138 & Organic carbon flow in a swamp-stream ecosystem & Mulholland P. J. & 1981 & Ecological Monographs & 51 & 3 & $307-322$ & $10.2307 / 2937276$ \\
\hline 139 & Sources, degradation and recycling of organic matter associated with sinking particles in Lake Michigan & Meyers P. A. and Eadie B. J. & 1993 & Organic Geochemistry & 20 & 1 & $47-56$ & 10.1016/0146-6380(93)90080-U \\
\hline 140 & Decomposition of Blue-Green Algal (Cyanobacterial) Blooms in Lake Mendota, Wisconsin & Fallon \& Brock & 1979 & Applied and Environmental Microbiology & 37 & 5 & $820-830$ & \\
\hline 141 & $\begin{array}{l}\text { The breakdown and decomposition of allochthonous and autochthonous plant litter in an oligotrophic lake } \\
\text { (Llyn Frongoch) }\end{array}$ & Hanlon R. D. G. & 1982 & Hydrobiologia & 88 & & $281-288$ & 10.1007/BF00008508 \\
\hline 142 & $\begin{array}{l}\text { Decomposition of dominant submerged macrophytes: implications for nutrient release in Myall Lake, NSW, } \\
\text { Australia }\end{array}$ & Shilla D. et al. & 2006 & Wetlands Ecology and Management & 14 & 5 & $427-433$ & $10.1007 / 511273-006-6294-9$ \\
\hline
\end{tabular}




\begin{tabular}{|c|c|c|c|c|c|c|c|c|}
\hline 143 & The influence of elevated nitrate concentration on rate of leaf decomposition in a stream & Meyer J. L. and Johnson C. & 1983 & Freshwater Biology & 13 & 2 & 177-183 & 10.1111/j.1365-2427.1983.tb00669.x \\
\hline 144 & Decomposition rate of plant material in the Parana Medio River (Argentina) & Hammerly J. et al. & 1989 & Hydrobiologia & 183 & & $179-184$ & $10.1007 / \mathrm{BF} 00007238$ \\
\hline 145 & Contribution of semi-arid forests to the climate system & Rotenberg E. and Yatir D. & 2010 & Science & 327 & 5964 & $451-454$ & 10.1126/science.1179998 \\
\hline 146 & $\mathrm{CO} 2$ balance of boreal, temperate, and tropical forests derived from a global database & Luyssaert S. et al. & 2007 & Global Change Biology & 13 & & 2509-2537 & 7 10.1111/j.1365-2486.2007.01439.x \\
\hline 147 & $\begin{array}{l}\text { Reciprocal fluxes of stream and riparian invertebrates in a coastal California basin with Mediterranean } \\
\text { climate }\end{array}$ & Rundio D. E. and Lindley S. T. & 2012 & Ecological research & 27 & 3 & $539-550$ & 10.1007/s11284-011-0920-6 \\
\hline 148 & Carbon stocks in a tropical dry forest in Brazil & Pereira Junior L. R. et al. & 2016 & Revista Ciência Agronômica & 47 & 1 & $32-40$ & 10.5935/1806-6690.20160004 \\
\hline 149 & Carbon Sequestration and Nutrient Reserves Under Different Land Use Systems & Aguiar M. I. et al. & 2014 & Revista Árvore & 38 & 1 & $81-93$ & $10.1590 / 50100-67622014000100008$ \\
\hline 150 & Effects of non-native Asian earthworm invasion on temperate forest and prairie soils in the Midwestern US & Qiu J. and Turner M. G. & 2016 & Biological Invasions & 19 & 1 & $73-88$ & $10.1007 /$ s10530-016-1264-5 \\
\hline 151 & $\begin{array}{l}\text { Differential responses of production and respiration to temperature and moisture drive the carbon balance } \\
\text { across a climatic gradient in New Mexico }\end{array}$ & Anderson-Teixeira K. et al. & 2011 & Global Change Biology & 17 & 1 & 410-424 & 10.1111/j.1365-2486.2010.02269.x \\
\hline 152 & Sequestration of $\mathrm{C}$ in Spanish deciduous oak forests & $\begin{array}{l}\text { Gallardo Lancho J. F. and } \\
\text { Gonzalez Hernandez M. I. }\end{array}$ & 2004 & Catena / Advances in Geoecology & 37 & & 341-351 & \\
\hline 153 & Carbon storage and emissions offset potential in an African dry forest, the Arabuko-Sokoke Forest, Kenya & Glenday J. & 2008 & Environmental Monitoring and Assessment & 142 & & $85-95$ & 10.1007/s10661-007-9910-0 \\
\hline 154 & $\begin{array}{l}\text { Drying and wetting of Mediterranean soils stimulates decomposition and carbon dioxide emission: the "Birch } \\
\text { effect". }\end{array}$ & Jarvis P. et al. & 2007 & Tree Physiology & 27 & 7 & 929-940 & 10.1093/treephys/27.7.929 \\
\hline 155 & Components, drivers and temporal dynamics of ecosystem respiration in a Mediterranean pine forest & Matteucci M. et al. & 2015 & Soil Biology and Biochemistry & 88 & & $224-235$ & 10.1016/j.soilbio.2015.05.017 \\
\hline 156 & $\begin{array}{l}\text { Carbon budget of tropical forests in Southeast Asia and the effects of deforestation : an approach using a } \\
\text { process-based model and field measurements }\end{array}$ & Adachi M. et al. & 2011 & Biogeosciences & 8 & & 2635-2647 & $10.5194 / \mathrm{bg}-8-2635-2011$ \\
\hline 157 & $\begin{array}{l}\text { Plant Ecology \& Diversity The productivity, allocation and cycling of carbon in forests at the dry margin of the } \\
\text { Amazon forest in Bolivia }\end{array}$ & Araujo-Murakami A. et al. & 2014 & Plant Ecology and Diversity & 7 & $1-2$ & $55-69$ & 10.1080/17550874.2013.798364 \\
\hline 158 & Seasonal and annual variation of carbon exchange in an evergreen Mediterranean forest in southern France & Allard V. et al. & 2008 & Global Change Biology & 14 & 4 & $714-725$ & 10.1111/j.1365-2486.2008.01539.x \\
\hline 159 & Inter-regional comparison of land-use effects on stream metabolism & Bernot M. J. et al. & 2010 & Freshwater Biology & 55 & 9 & 1874-1890 & 10.1111/j.1365-2427.2010.02422.x \\
\hline 160 & $\begin{array}{l}\text { The seasonal cycle of productivity, metabolism and carbon dynamics in a wet aseasonal forest in north-west } \\
\text { Amazonia (Iquitos, Peru) }\end{array}$ & Aguila-Pasquel del J. et al. & 2014 & Plant Ecology and Diversity & 7 & $1-2$ & 71-83 & 10.1080/17550874.2013.798365 \\
\hline 161 & Carbon allocation in a Bornean tropical rainforest without dry seasons & Katayama A. et al. & 2013 & Journal of plant research & 126 & 4 & 505-515 & 10.1007/s10265-012-0544-0 \\
\hline 162 & Response of net ecosystem productivity of three boreal forest stands to drought & Kljun N. et al. & 2007 & Ecosystems & 10 & 6 & 1039-1055 & 10.1007/s10021-007-9088-x \\
\hline 163 & $\begin{array}{l}\text { The productivity, metabolism and carbon cycle of two lowland tropical forest plots in south-western } \\
\text { Amazonia, Peru }\end{array}$ & Malhi Y. et al. & 2014 & Plant Ecology and Diversity & 7 & $1-2$ & 85-105 & 10.1080/17550874.2013.820805 \\
\hline 164 & Carbon stocks and dynamics at different successional stages in an Afromontane tropical forest & Nyirambangutse B. et al. & 2016 & Biogeosciences Discussions & 14 & & 1285-1303 & B 10.5194/bg-14-1285-2017 \\
\hline 165 & Biometric and Micrometeorological Measurements of Tropical Forest Carbon Balance & Miller S. D. et al. & 2014 & Ecological Applications & 14 & 4 & S114-S126 & $10.1890 / 02-6005$ \\
\hline 166 & $\begin{array}{l}\text { Land-atmosphere carbon and water flux relationships to vapor pressure deficit, soil moisture, and stream } \\
\text { flow }\end{array}$ & Mitchell S. R. et al. & 2015 & Agricultural and Forest Meteorology & 208 & & $108-117$ & 10.1016/j.agrformet.2015.04.003 \\
\hline 167 & Spatial - temporal variability of terrestrial vegetation productivity in the Yangtze River Basin during & Zhang Y. et al. & 2014 & Journal of plant ecology & 7 & 1 & $10-23$ & 10.1093/jpe/rtt025 \\
\hline 168 & $\begin{array}{l}\text { Scaling net ecosystem production and net biome production over a heterogeneous region in the western } \\
\text { United States }\end{array}$ & Turner D. P. et al. & 2007 & Biogeosciences & 4 & 2 & 1093-1135 & 10.5194/bgd-4-1093-2007 \\
\hline 169 & $\begin{array}{l}\text { Modelling the climatic drivers determining photosynthesis and carbon allocation in evergreen Mediterranean } \\
\text { forests using multiproxy long time series }\end{array}$ & Gea-Izquierdo G. et al. & 2015 & Biogeosciences & 12 & & 3695-3712 & $10.5194 / \mathrm{bg}-12-3695-2015$ \\
\hline 170 & $\begin{array}{l}\text { Landscape and Urban Planning Environmental control over seasonal variation in carbon fluxes of an urban } \\
\text { temperate forest ecosystem }\end{array}$ & Xie J. et al. & 2015 & Landscape and Urban Planning & 142 & & $63-70$ & 10.1016/j.landurbplan.2015.04.011 \\
\hline 171 & Seasonal and inter-annual variations in net ecosystem exchange of two old-growth forests in southern China & Yan J. et al. & 2013 & Agricultural and Forest Meteorology & $182-183$ & & $257-265$ & 10.1016/j.agrformet.2013.03.002 \\
\hline 172 & Upscaling carbon fluxes over the Great Plains grasslands: Sinks and sources & Zhang L. et al. & 2011 & $\begin{array}{l}\text { Journal of Geophysical Research: } \\
\text { Biogeosciences }\end{array}$ & 116 & 1 & G00J03 & 10.1029/2010JG001504 \\
\hline 173 & Airborne scanning LiDAR in a double sampling forest carbon inventory & Stephens P. R. et al. & 2012 & Remote Sensing of Environment & 117 & & $348-357$ & 10.1016/j.rse.2011.10.009 \\
\hline 174 & Seasonal changes in photosynthesis, respiration and NEE of a mixed temperate forest & Carrara A. et al. & 2004 & Agricultural and Forest Meteorology & 126 & $1-2$ & $15-31$ & 10.1016/j.agrformet.2004.05.002 \\
\hline 175 & Primary productivity of natural grass ecosystems of the tropics: A reappraisal & Long S. P. et al. & 1989 & Plant and Soil & 115 & 2 & $155-166$ & $10.1007 / B F 02202584$ \\
\hline 176 & A Meta-analysis of Climatic and Chemical Controls on Leaf Litter Decay Rates in Tropical Forests & Waring B. G. & 2012 & Ecosystems & 15 & 6 & 999-1009 & 10.1007/s10021-012-9561-z \\
\hline 177 & Carbon dioxide exchange in a tropical wet grassland & Meirelles M. L. et al. & 2015 & Wetlands Ecology and Management & 23 & 5 & $817-826$ & 10.1007/s11273-015-9421-7 \\
\hline 178 & The utility of the eddy covariance techniques as a tool in carbon accounting: tropical savanna as a case study & Hutley L. B. et al. & 2005 & Australian Journal of Botany & 53 & 7 & $663-675$ & 10.1071/bt04147 \\
\hline 179 & Decomposition and nutrient release patterns of mistletoe litters in a semi-arid savanna, southwest Zimbabwe & Ndagurwa H. G. T. et al. & 2015 & Austral Ecology & 40 & 2 & 178-185 & 10.1111/aec.12191 \\
\hline
\end{tabular}




\begin{tabular}{|c|c|c|c|c|c|c|c|c|}
\hline 180 & $\begin{array}{l}\text { Litterfall and nutrient return in a semi-arid southern African savanna woodland dominated by } \\
\text { Colophospermum mopane }\end{array}$ & Mlambo D. and Nyathi P. & 2008 & Plant Ecology & 196 & 1 & $101-110$ & 10.1007/s11258-007-9337-2 \\
\hline 181 & Inhibition of nitrification alters carbon turnover in the Patagonian steppe & Austin A. T. et al. & 2006 & Ecosystems & 9 & 8 & 1257-1265 & $10.1007 / s 10021-005-0039-0$ \\
\hline 182 & Soil carbon pools and fluxes after land conversion in a semiarid shrub-steppe ecosystem & Cochran R. L. et al. & 2007 & Biology and Fertility of Soils & 43 & 4 & 479-489 & 10.1007/s00374-006-0126-1 \\
\hline 183 & $\begin{array}{l}\text { Plant functional diversity and carbon storage - an empirical test in semi-arid forest ecosystems } \\
\end{array}$ & Conti G. and Diaz S. & 2013 & Journal of Ecology & 101 & 1 & $18-28$ & $10.1111 / 1365-2745.12012$ \\
\hline 184 & $\begin{array}{l}\text { Decomposition and nutrient dynamics of Quercus pubescens leaf litter in a naturally enriched CO2 } \\
\text { Mediterranean ecosystem }\end{array}$ & Cotrufo M. F. et al. & 1999 & Functional Ecology & 13 & 3 & 343-351 & 10.1046/j.1365-2435.1999.00328.x \\
\hline 185 & The Role of Termites in the Decomposition of Wood and Leaf Litter in the Southern Guinea Savanna of Nigeria & a Collins N. M. & 1981 & Oecologia & 51 & 3 & 389-399 & 10.1007/BF00540911 \\
\hline 186 & Decomposition of grasses and forbs in coastal savanna of southern Somalia & Thurow L. T. & 1989 & African Journal of Ecology & 27 & 3 & $201-206$ & 10.1111/j.1365-2028.1989.tb01013.x \\
\hline 187 & Litter production and decomposition patterns in seral grasslands at Cherrapunji in north-eastern India & $\begin{array}{l}\text { Ram S. C. and Ramakrishnan P. } \\
\text { S. }\end{array}$ & 1988 & Pedobiologia & 32 & $1-2$ & $65-76$ & \\
\hline 188 & Environmental controls on the spatial variability of savanna productivity in the Northern Territory, Australia & Kanniah K. D. et al. & 2011 & Agriculture and Forest Meteorology & 151 & 11 & 1429-1439 & 10.1016/j.agrformet.2011.06.009 \\
\hline 189 & Dynamics in carbon exchange fluxes for a grazed semi-arid savanna ecosystem in West Africa & Tagesson T. et al. & 2015 & Agriculture, Ecosystems and Environment & 205 & & $15-24$ & 10.1016/j.agee.2015.02.017 \\
\hline 190 & Phenology and gross primary production of two dominant savanna woodland ecosystems in Southern Africa & Jin C. et al. & 2013 & Remote Sensing of Environment & 135 & $\begin{array}{c}\text { Marc } \\
\mathrm{h}\end{array}$ & 189-201 & 10.1016/j.rse.2013.03.033 \\
\hline 191 & $\begin{array}{l}\text { Nutrient addition does not enhance leaf decomposition in a Southeastern Brazilian stream (Espinhaço } \\
\text { mountain range) }\end{array}$ & Abelho M. et al. & 2010 & Brazilian Journal of Biology & 70 & 3 & $747-754$ & $10.1590 / \mathrm{S} 1519-69842010000400007$ \\
\hline 192 & Does leaf quality mediate the stimulation of leaf breakdown by phosphorus in Neotropical streams? & Ardon M. et al. & 2010 & Freshwater Biology & 51 & 4 & $618-633$ & 10.1111/j.1365-2427.2006.01515.x \\
\hline 193 & The Effect of Macroinvertebrate Exclusion on Leaf Breakdown Rates in a Tropical Headwater Stream & Wright and Covich & 2005 & Biotropica & 37 & 3 & $403-408$ & 10.1111/j.1744-7429.2005.00053.x \\
\hline 194 & Soil-Litter Mixing Accelerates Decomposition in a Chihuahuan Desert Grassland & Hewins D. B. et al. & 2013 & Ecosystems & 16 & 2 & $183-195$ & $10.1007 / 510021-012-9604-5$ \\
\hline 195 & Decomposition of dominant plant species litter in a semi-arid grassland & Koukoura Z. et al. & 2003 & Applied Soil Ecology & 23 & 1 & $13-23$ & 10.1016/S0929-1393(03)00006-4 \\
\hline 196 & Leaf breakdown in a natural open tropical stream & Alvim A. et al. & 2015 & Journal of Limnology & 74 & 2 & $248-260$ & 10.4081/jlimnol.2014.982 \\
\hline 197 & $\begin{array}{l}\text { Litter decomposition in a temperate and a tropical stream: the effects of species mixing, litter quality and } \\
\text { shredders }\end{array}$ & Bruder A. et al. & 2014 & Freshwater Biology & 59 & 3 & 438-449 & 10.1111/fwb.12276 \\
\hline 198 & Breakdown of leaf litter in a neotropical stream & Mathuriau C. and Chauvet E. & 2002 & J. of the North Am. Benth. Soc. & 21 & 3 & $384-396$ & $10.2307 / 1468477$ \\
\hline 199 & Macroinvertebrates and the Processing of Leaf Litter in a Tropical Stream & Benstead J. P. & 1996 & Biotropica & 28 & 3 & $367-375$ & $10.2307 / 2389200$ \\
\hline 200 & Leaf-litter breakdown in 3 streams in temperate, Mediterranean, and tropical Cerrado climates & Goncalves J. F. et al. & 2006 & J. of the North Am. Benth. Soc. & 25 & 2 & $344-355$ & $\begin{array}{l}\text { http://dx.doi.org/10.1899/0887- } \\
\text { 3593(2006)25[344:LBISIT]2.0.CO_2 }\end{array}$ \\
\hline 201 & Evaluation of macrofaunal effects on leaf litter breakdown rates in aquatic and terrestrial habitats & Ribas A. D. de A. et al. & 2006 & Austral Ecology & 31 & 6 & $783-790$ & 10.1111/j.1442-9993.2006.01640.x \\
\hline 202 & Relationships of Stream Invertebrate Communities To Deforestation in Eastern Madagascar & Benstead J. P. et al. & 2003 & Ecological Applications & 13 & 5 & 1473-1490 & $10.1890 / 02-5125$ \\
\hline 203 & Modeling C and N flows through a stream food web: an inverse approach & Larned S. T. et al. & 2008 & J. of the North Am. Benth. Soc. & 27 & 3 & $674-689$ & $10.1899 / 07-134.1$ \\
\hline 204 & $\begin{array}{l}\text { Seasonal dynamics of benthic detritus and associated macroinvertebrate communities in a neotropical } \\
\text { stream }\end{array}$ & Mathuriau C. et al. & 2008 & $\begin{array}{l}\text { Fundamental and Applied Limnology Archiv } \\
\text { für Hydrobiologie }\end{array}$ & 171 & 4 & 323-333 & 10.1127/1863-9135/2008/0171-0323 \\
\hline 205 & Organic Matter Processing in Tropical Streams & Wantzen K. M. et al. & 2008 & In: Tropical Stream Ecology (book) & & & $43-64$ & doi:10.1016/B978-012088449-0.50005-4 \\
\hline 206 & $\begin{array}{l}\text { Impacts of Benthic Coarse Particulate Organic Matter variations on Macroinvertebrate Density and Diversity } \\
\text { in the Njoro River, A Kenyan Highland Stream }\end{array}$ & Mbaka J. G. et al. & 2014 & Journal of East African Natural History & 103 & 1 & 39-48 & 10.2982/028.103.0101 \\
\hline 207 & Ecosystem measures of river health and their response to riparian and catchment degradation & Bunn S. E. et al. & 1999 & Freshwater Biology & 41 & 2 & $333-345$ & 10.1046/j.1365-2427.1999.00434.x \\
\hline 208 & Metabolism of a tropical rainforest stream & Ortiz-Zayas J. R. et al. & 2005 & J. of the North Am. Benth. Soc. & 24 & 4 & $769-783$ & 10.1899/03-094.1 \\
\hline 209 & Seasonally variable riverine production in the Venezuelan llanos & Cotner J. B. et al. & 2006 & J. of the North Am. Benth. Soc. & 25 & 1 & $171-184$ & $\begin{array}{l}\text { 10.1899/0887- } \\
\text { 3593(2006)25[171:SVRPIT]2.0.CO_2 }\end{array}$ \\
\hline 210 & $\begin{array}{l}\text { Seasonal changes in light availability modify the temperature dependence of ecosystem metabolism in an } \\
\text { arctic stream }\end{array}$ & Huryn A. D. et al. & 2014 & Ecology & 95 & 10 & 2826-2839 & $10.1890 / 13-1963.1$ \\
\hline 211 & Inter-biome comparison of factors controlling stream metabolism & Mulholland P. J. et al. & 2001 & Freshwater Biology & 46 & & 1503-1517 & 10.1046/j.1365-2427.2001.00773.x \\
\hline 212 & Composition and dynamics of allochthonous organic matter inputs and benthic stock in a Brazilian stream & França J. S. et al. & 2009 & Marine and Freshwater Research & 60 & 10 & $990-998$ & $10.1071 / \mathrm{MF} 08247$ \\
\hline 213 & Detritivores in Kenyan highland streams: more evidence for the paucity of shredders in the tropics? & Dobson M. et al. & 2002 & Freshwater Biology & 47 & 5 & $909-919$ & 10.1046/j.1365-2427.2002.00818.x \\
\hline 214 & $\begin{array}{l}\text { Influence of watershed-climate interactions on stream temperature, sediment yield, and metabolism along a } \\
\text { land use intensity gradient in Indonesian Borneo }\end{array}$ & Carlson K. M. et al. & 2014 & $\begin{array}{l}\text { Journal of Geophysical Research: } \\
\text { Biogeosciences }\end{array}$ & 119 & 6 & 1110-1128 & $10.1002 / 2013 \mathrm{JG} 002516$ \\
\hline 215 & Structure and Production of a Benthic Insect Assemblage in a Neotropical Stream & Ramírez A. and Pringle C. M. & 1998 & J. of the North Am. Benth. Soc. & 17 & 4 & $443-463$ & $10.2307 / 1468365$ \\
\hline 216 & $\begin{array}{l}\text { Biodiversity and ecosystem function in species-poor communities: community structure and leaf litter } \\
\text { breakdown in a Pacific island stream }\end{array}$ & Benstead J. P. et al. & 2009 & J. of the North Am. Benth. Soc. & 28 & 2 & 454-465 & http://dx.doi.org/10.1899/07-081.1 \\
\hline 217 & Detritivorous fish indirectly reduce insect secondary production in a tropical river & Hall R. O. et al. & 2011 & Ecosphere & 2 & 12 & Art.135 & 10.1890/ES11-00042.1 \\
\hline
\end{tabular}




\begin{tabular}{|c|c|c|c|c|c|c|c|c|}
\hline 218 & $\begin{array}{l}\text { Impacts of agricultural land use on ecosystem structure and whole-stream metabolism of tropical Cerrado } \\
\text { streams }\end{array}$ & Gücker B. et al. & 2009 & Freshwater Biology & 54 & & 2069-2085 & 10.1111/j.1365-2427.2008.02069.x \\
\hline 219 & Differential Decomposition of Leaves in Grassland and Gallery Forest Reaches of Kings Creek & Hooker K. L. et al. & 1987 & $\begin{array}{l}\text { Transactions of the Kansas Academy of } \\
\text { Science }\end{array}$ & 90 & $1-2$ & $17-24$ & $10.2307 / 3628107$ \\
\hline 220 & Dissolved organic matter release in a Posidonia oceanica meadow & Barrón C. and Duarte C. M. & 2009 & Marine Ecology Progress Series & 374 & & $75-84$ & $10.3354 / \mathrm{meps} 07715$ \\
\hline 221 & Community metabolism and carbon budget along a gradient of seagrass (Cymodocea nodosa) colonization & Barrón C. et al. & 2009 & Limnology and Oceanography & 49 & 5 & 1642-1651 & 10.4319/lo.2004.49.5.1642 \\
\hline 222 & Factors controlling net ecosystem metabolism in U.S. estuaries & Caffrey J. M. & 2004 & Estuaries & 27 & 1 & $90-101$ & $10.1007 / \mathrm{BF} 02803563$ \\
\hline 223 & $\begin{array}{l}\text { Primary production of seagrass beds in South Sulawesi (Indonesia): a comparison of habitats, methods and } \\
\text { species }\end{array}$ & Erftemeijer P. L. A. et al. & 1993 & Aquatic Botany & 46 & & $67-90$ & $10.1016 / 0304-3770(93) 90065-5$ \\
\hline 224 & $\begin{array}{l}\text { Opposing effects of substrate quality and site factors on forest floor turnover rates: an example from the } \\
\text { tropics }\end{array}$ & $\begin{array}{l}\text { Rahman M. M. and Tsukamoto } \\
\text { J. }\end{array}$ & 2014 & Forestry & 88 & 2 & 190-199 & 10.1093/forestry/cpu043 \\
\hline 225 & $\begin{array}{l}\text { Effects of manipulated herbivore inputs on nutrient flux and decomposition in a tropical rainforest in Puerto } \\
\text { Rico }\end{array}$ & Schowalter T. D. et al. & 2011 & Oecologia & 167 & 4 & $1141-1149$ & $10.1007 / 500442-011-2056-3$ \\
\hline 226 & $\begin{array}{l}\text { Litter decomposition rates in relation to litter stocks in boreal coniferous forests along climatic and soil } \\
\text { fertility gradients }\end{array}$ & Hilli S. et al. & 2010 & Applied Soil Ecology & 46 & 2 & $200-208$ & 10.1016/j.apsoil.2010.08.012 \\
\hline 227 & $\begin{array}{l}\text { An experimental comparison of chemical traits and litter decomposition rates in a diverse range of subarctic } \\
\text { bryophyte, lichen and vascular plant species }\end{array}$ & Lang S. L. et al. & 2009 & Journal of Ecology & 97 & 5 & $886-900$ & 10.1111/j.1365-2745.2009.01538.x \\
\hline 228 & $\begin{array}{l}\text { Decomposition of pure and mixed foliage litter in a young lodgepole pine - Sitka alder stand in the central } \\
\text { interior of British Columbia }\end{array}$ & Sanborn P. T. and Brockley R. P. & 2009 & Canadian Journal of Forest Research & 39 & 11 & $2257-2262$ & 10.1139/x09-122 \\
\hline 229 & $\begin{array}{l}\text { Decomposition of Scots pine fine woody debris in boreal conditions: Implications for estimating carbon pools } \\
\text { and fluxes }\end{array}$ & Vávřová P. et al. & 2009 & Forest Ecology and Management & 257 & 2 & 401-412 & 10.1016/j.foreco.2008.09.017 \\
\hline 230 & $\begin{array}{l}\text { Breakdown and macroinvertebrate and fungal colonization of alder, birch, and willow leaves in a boreal forest } \\
\text { stream }\end{array}$ & Haapala A. et al. & 2001 & J. of the North Am. Benth. Soc. & 20 & 3 & $395-407$ & $10.2307 / 1468037$ \\
\hline 231 & The timing of winter-growing shredder species and leaf litter turnover rate in an oligotrophic lake, SE Sweden & Bohman I. M. and Herrmann J. & 2006 & Hydrobiologia & 556 & 1 & 99-108 & $10.1007 /$ s10750-005-1052-1 \\
\hline 232 & $\begin{array}{l}\text { Remote estimation of gross primary production in maize and support for a new paradigm based on total crop } \\
\text { chlorophyll content }\end{array}$ & Peng Y. et al. & 2011 & Remote Sensing of Environment & 115 & 4 & 978-989 & 10.1016/j.rse.2010.12.001 \\
\hline 233 & Benthic respiration and nitrogen release in Buzzards Bay, Massachusetts & Banta G. T. et al. & 1995 & Journal of Marine Research & 53 & 1 & $107-135$ & $10.1357 / 0022240953213287$ \\
\hline 234 & Metabolism of a Desert Stream & Busch D. E. and Fisher S. G. & 1981 & Freshwater Biology & 11 & May & $301-307$ & 10.1111/j.1365-2427.1981.tb01263.x \\
\hline 235 & Flow extremes and benthic organic matter shape the metabolism of a headwater Mediterranean stream & Acuña V. et al. & 2004 & Freshwater Biology & 49 & 7 & $960-971$ & 10.1111/j.1365-2427.2004.01239.x \\
\hline 236 & $\begin{array}{l}\text { Interrelationships Among Shrub Encroachment, Land Management, and Litter Decomposition in a } \\
\text { Semidesert Grassland }\end{array}$ & Throop H. L. and Archer S. R. & 2007 & Ecological Applications & 17 & 6 & 1809-1823 & 10.1890/06-0889.1 \\
\hline 237 & $\begin{array}{l}\text { In situ litter decomposition and litter quality in a Mojave Desert ecosystem: effects of elevated atmospheric } \\
\text { CO2 and interannual climate variability }\end{array}$ & Weatherly H. E. et al. & 2003 & Global Change Biology & 9 & 8 & 1223-1233 & 10.1046/j.1365-2486.2003.00653.x \\
\hline 238 & Organic Matter Flow in Stream Food Webs With Reduced Detrital Resource Base & Hall R. O. et al. & 2000 & Ecology & 81 & 12 & 3445-3463 & 10.2307/177506 \\
\hline 239 & $\begin{array}{l}\text { Trophic model of the subtropical headwater stream habitat of formosan landlocked salmon oncorhynchus } \\
\text { formosanus }\end{array}$ & Lin H.J. et al. & 2012 & Aquatic Biology & 17 & 3 & $269-283$ & 10.3354/ab00481 \\
\hline 240 & Macroinvertebrate production and trophic structure in a tallgrass prairie headwater stream & $\begin{array}{l}\text { Stagliano D. M. and Whiles M. } \\
\text { R. }\end{array}$ & 2002 & J. of the North Am. Benth. Soc. & 21 & 1 & $97-113$ & $10.2307 / 1468303$ \\
\hline 241 & Secondary Production Estimates Of Benthic Insects In 3 Cold Desert Streams & Gaines W. L. et al. & 1992 & Great Basin Naturalist & 52 & 1 & $11-24$ & $10.2307 / 41712690$ \\
\hline 242 & Effects of Disturbance on Benthic Functional Structure and Production in Mountain Streams & Lugthart J. G. and Wallace B. J. & 1992 & J. of the North Am. Benth. Soc. & 11 & 2 & $138-164$ & $10.2307 / 1467381$ \\
\hline 243 & Trophic basis of invertebrate production in 2 streams at the Hubbard Brook Experimental Forest & Hall R. O. et al. & 2001 & J. of the North Am. Benth. Soc. & 20 & 3 & $432-447$ & $10.2307 / 1468040$ \\
\hline 244 & Secondary Production and Trophic Relationships in a Spring Invertebrate Community & Iversen T.M. & 1988 & Limnology and Oceanography & 33 & 4 & $582-592$ & 10.4319/lo.1988.33.4.0582 \\
\hline 245 & Secondary Production and Organic Matter Processing by Collector Macroinvetebrates in a Desert Stream & Fisher S. G. and Gray L. J. & 1983 & Ecology & 64 & 5 & 1217-1224 & 10.2307/1937830 \\
\hline 246 & Secondary Production in Inland Waters & Waters T. F. & 1977 & Advances in Ecological Research & 10 & $\mathrm{C}$ & $91-164$ & $10.1016 / 50065-2504(08) 60235-4$ \\
\hline 247 & $\begin{array}{l}\text { Benthic and Pelagic Secondary Production in Lake Erie after the Invasion of Dreissena spp. with Implications } \\
\text { for Fish Production }\end{array}$ & Johannsson O. E. et al. & 2000 & Journal of Great Lakes Research & 26 & 1 & $31-54$ & 10.1016/S0380-1330(00)70671-X \\
\hline 248 & $\begin{array}{l}\text { Litter carbon dynamics analysis in forests in an arid ecosystem with a model incorporating the physical } \\
\text { removal of litter }\end{array}$ & Kumada S. et al. & 2008 & Ecological Modelling & 215 & $1-3$ & 190-199 & 10.1016/j.ecolmodel.2008.02.022 \\
\hline 249 & Quantifying carbon budgets of conifer Mediterranean forest ecosystems, Turkey & Evrendilek F. et al. & 2006 & Environmental Monitoring and Assessment & 119 & & $527-543$ & $10.1007 / 510661-005-9041-4$ \\
\hline 250 & Spatial heterogeneity of ecosystem carbon fluxes in a broadleaved forest in Northern Germany & Kutsch W. L. et al. & 2005 & Global Change Biology & 11 & 1 & $70-88$ & 10.1111/j.1365-2486.2004.00884.x \\
\hline 251 & Production and Litter Decomposition Rate in Acahuales of Deciduous Tropical Forest in Chiapas & Rivera Vasquez R. et al. & 2013 & Revista Mexicana de Ciencias Forestales & 4 & 20 & $20-30$ & $\begin{array}{l}\text { http://www.redalyc.org/articulo.oa?id=634 } \\
33994003\end{array}$ \\
\hline
\end{tabular}




\begin{tabular}{|c|c|c|c|c|c|c|c|c|}
\hline 252 & Rate of litter decomposition and microbial activity in an area of caatinga & Souto P. C. et al. & 2013 & Cerne & 19 & 4 & $559-565$ & $10.1590 / 50104-77602013000400005$ \\
\hline 253 & $\begin{array}{l}\text { Tree Species Effect on Litter Decomposition and Nutrient Release in Mediterranean Oak Forests Changes } \\
\text { Over Time }\end{array}$ & Aponte C. et al. & 2012 & Ecosystems & 15 & 7 & 1204-1218 & 310.1007/s10021-012-9577-4 \\
\hline 254 & Role of desert annuals in nutrient flow in arid area of Northwestern China: A nutrient reservoir and provider & Chen B.-M. et al. & 2009 & Plant Ecology & 201 & & 401-409 & 10.1007/s11258-008-9526-7 \\
\hline 255 & Litter quality and decomposition in a CO2-enriched Mediterranean forest ecosystem & De Angelis P. et al. & 2000 & Plant and Soil & 224 & 1 & $31-41$ & 10.1023/A:1004790328560 \\
\hline 256 & Regional patterns and controls of leaf decomposition in Australian tropical rainforests & Parsons S. A. et al. & 2012 & Austral Ecology & 37 & 7 & $845-854$ & 10.1111/j.1442-9993.2011.02347.x \\
\hline 257 & Seasonality of ecosystem respiration and gross primary production as derived from FLUXNET measurements & Falge E. et al. & 2002 & Agricultural and Forest Meteorology & 113 & & $53-74$ & $10.1016 / 50168-1923(02) 00102-8$ \\
\hline 258 & $\begin{array}{l}\text { Estimation of carbon stock under different management regimes of tropical forest in the Terai Arc Landscape, } \\
\text { Nepal }\end{array}$ & Gurung M. B. et al. & 2015 & Forest Ecology and Management & 365 & & $144-152$ & 10.1016/j.foreco.2015.07.024 \\
\hline 259 & $\begin{array}{l}\text { Carbon stocks in different carbon pools of a tropical lowland forest and a montane forest with varying } \\
\text { topography }\end{array}$ & Jeyanny V. et al. & 2014 & Journal of Tropical Forest Science & 26 & 4 & $560-571$ & http://www.frim.gov.my/.../560-571.pdf \\
\hline 260 & Simulated annual carbon fluxes of grassland ecosystems in extremely arid conditions & Zhang $\mathrm{N}$. et al. & 2009 & Ecological Research & 24 & 1 & $185-206$ & $10.1007 / 511284-008-0497-x$ \\
\hline 261 & Ecosystem metabolism controls nitrogen uptake in streams in Grand Teton National Park, Wyoming & Hall R. O. and Tank J. L. & 2003 & Limnology and Oceanography & 48 & 3 & $1120-1128$ & 10.4319/lo.2003.48.3.1120 \\
\hline 262 & Hot spots for carbon emissions from Mediterranean fluvial networks during summer drought & Gómez-Gener L. et al. & 2015 & Biogeochemistry & 125 & 3 & $409-426$ & 10.1007/s10533-015-0139-7 \\
\hline 263 & $\begin{array}{l}\text { Ecohydrological impacts of woody-plant encroachment: Seasonal patterns of water and carbon dioxide } \\
\text { exchange within a semiarid riparian environment }\end{array}$ & Scott R. L. et al. & 2006 & Global Change Biology & 12 & 2 & $311-324$ & 10.1111/j.1365-2486.2005.01093.x \\
\hline 264 & Influence of land use on stream ecosystem function in a Mediterranean catchment & Von Schiller D. et al. & 2008 & Freshwater Biology & 53 & 12 & $2600-2612$ & 10.1111/j.1365-2427.2008.02059.x \\
\hline 265 & $\begin{array}{l}\text { High secondary production in a Coastal Plain river is dominated by snag invertebrates and fuelled mainly by } \\
\text { amorphous detritus }\end{array}$ & Benke A. C. and Wallace J. B. & 2015 & Freshwater Biology & 60 & 2 & $236-255$ & 10.1111/fwb.12460 \\
\hline 266 & Bacterial production in the carbon flow of a central European stream, the Breitenbach & Marxsen J. & 2006 & Freshwater Biology & 51 & 10 & 1838-1861 & 10.1111/j.1365-2427.2006.01620.x \\
\hline 267 & Stream Organic Matter Budgets : An Introduction & Webster J. R. and Meyer J. L. & 1997 & J. of the North Am. Benth. Soc. & 16 & 1 & $3-13$ & $10.2307 / 1468223$ \\
\hline 268 & Autotropy in Stream Ecosystems & Minshall W. G. & 1978 & BioScience & 28 & 12 & $767-771$ & $10.2307 / 1307250$ \\
\hline 269 & Leaf litter as a possible food source for chironomids (Diptera) in Brazilian and Portuguese headwater streams & Callisto M. et al. & 2007 & Revista Brasileira de Zoologia & 24 & 2 & $442-448$ & 10.1590/50101-81752007000200023 \\
\hline 270 & $\begin{array}{l}\text { Benthic community metabolism in four temperate stream systems: an inter-biome comparison and } \\
\text { evaluation of the river continuum concept }\end{array}$ & Bott T. L. et al. & 1985 & Hydrobiologia & 123 & 1 & $3-45$ & 10.1007/BF00006613 \\
\hline 271 & Integrating aquatic carbon fluxes in a boreal catchment carbon budget & Jonsson A. et al. & 2007 & Journal of Hydrology & 334 & $1-2$ & $141-150$ & 10.1016/j.jhydrol.2006.10.003 \\
\hline 272 & $\begin{array}{l}\text { A comparison of the carbon balances of a natural lake (L. Örträsket) and a hydroelectric reservoir (L. } \\
\text { Skinnmuddselet) in northern Sweden }\end{array}$ & Åberg J. et al. & 2004 & Water Research & 38 & 3 & $531-538$ & 10.1016/j.watres.2003.10.035 \\
\hline 273 & Litter quality effects on soil carbon and nitrogen dynamics in temperate alley cropping systems & $\begin{array}{l}\text { Mungai N. W. and Motavalli P. } \\
\text { P. }\end{array}$ & 2006 & Applied Soil Ecology & 31 & $1-2$ & $32-42$ & 10.1016/j.apsoil.2005.04.009 \\
\hline 274 & Arctic lakes and streams as gas conduits to the atmosphere: implications for tundra carbon budgets. & Kling G. W. et al. & 1991 & Science & 251 & 4991 & 298-301 & 10.1126/science.251.4991.298 \\
\hline 275 & $\begin{array}{l}\text { Relationships between ecosystem metabolism, benthic macroinvertebrate densities, and environmental } \\
\text { variables in a sub-arctic Alaskan river }\end{array}$ & Benson E. R. et al. & 2013 & Hydrobiologia & 701 & 1 & $189-207$ & $10.1007 / \mathrm{s} 10750-012-1272-0$ \\
\hline 276 & $\begin{array}{l}\text { Short-term and long-term carbon dynamics in a northern peatland-stream-lake continuum: A catchment } \\
\text { approach }\end{array}$ & Juutinen S. et al. & 2013 & $\begin{array}{l}\text { Journal of Geophysical Research: } \\
\text { Biogeosciences }\end{array}$ & 118 & 1 & 171-183 & 10.1002/jgrg.20028 \\
\hline 277 & The carbon budget of newly established temperate grassland depends on management intensity & Ammann C. et al. & 2007 & Agriculture, Ecosystems and Environment & 121 & & $05-20$ & 10.1016/j.agee.2006.12.002 \\
\hline 278 & $\begin{array}{l}\text { Seasonal and interannual variation in carbon dioxide exchange and carbon balance in a northern temperate } \\
\text { grassland }\end{array}$ & Flanagan L. B. et al. & 2002 & Global Change Biology & 8 & 7 & $599-615$ & 10.1046/j.1365-2486.2002.00491.x \\
\hline 279 & $\begin{array}{l}\text { Partitioning European grassland net ecosystem CO2 exchange into gross primary productivity and ecosystem } \\
\text { respiration using light response function analysis }\end{array}$ & Gilmanov T. G. et al. & 2007 & Agriculture, Ecosystems and Environment & 121 & & 93-120 & 10.1016/j.agee.2006.12.008 \\
\hline 280 & $\begin{array}{l}\text { Carbon dynamics of temperate grassland ecosystems in China from } 1951 \text { to 2007: An analysis with a process- } \\
\text { based biogeochemistry model }\end{array}$ & Sui X. and Zhou G. & 2013 & Environmental Earth Sciences & 68 & 2 & $521-533$ & 10.1007/s12665-012-1756-2 \\
\hline 281 & $\begin{array}{l}\text { Seasonal carbon dynamics and the effects of manure application on carbon budget of a managed grassland in } \\
\text { a temperate, humid region in Japan }\end{array}$ & Matsuura S. et al. & 2014 & Grassland Science & 60 & 2 & 76-91 & 10.1111/grs.12042 \\
\hline 282 & Carbon dynamics and budget in a Zoysia japonica grassland, central Japan & Dhital D. et al. & 2010 & Journal of Plant Research & 123 & 4 & $519-530$ & 10.1007/s10265-009-0289-6 \\
\hline 283 & Inter-annual variability in carbon dioxide exchange of an oak/grass savanna and open grassland in California & Ma S. et al. & 2007 & Agricultural and Forest Meteorology & 147 & 3-4 & $157-171$ & 10.1016/j.agrformet.2007.07.008 \\
\hline 284 & $\begin{array}{l}\text { Warming alters potential enzyme activity but precipitation regulates chemical transformations in grass litter } \\
\text { exposed to simulated climatic changes }\end{array}$ & Suseela V. et al. & 2014 & Soil Biology and Biochemistry & 75 & & $102-112$ & 10.1016/j.soilbio.2014.03.022 \\
\hline 285 & Environmental influences on carbon dioxide fluxes over three grassland ecosystems in China & Fu Y. et al. & 2009 & Biogeosciences & 6 & 12 & 2879-2893 & 10.5194/bg-6-2879-2009 \\
\hline 286 & $\begin{array}{l}\text { Temperature and biomass influences on interannual changes in } \mathrm{CO} 2 \text { exchange in an alpine meadow on the } \\
\text { Qinghai-Tibetan Plateau }\end{array}$ & Kato C. et al. & 2006 & Global Change Biology & 12 & 7 & $1285-1298$ & 10.1111/j.1365-2486.2006.01153.x \\
\hline 287 & Characteristics of the gas exchange of a tropical rain forest in Peninsular Malaysia & Takanashi S. et al. & 2005 & Phyton; annales rei botanicae & 45 & 4 & $61-66$ & \\
\hline
\end{tabular}




\begin{tabular}{|c|c|c|c|c|c|c|c|c|}
\hline 288 & Three years of carbon and energy fluxes from Japanese evergreen broad-leaved forest & Kosugi Y. et al. & 2005 & Agricultural and Forest Meteorology & 132 & 3-4 & $329-343$ & 10.1016/j.agrformet.2005.08.010 \\
\hline 289 & Seasonal controls on the exchange of carbon and water in an Amazonian rain forest & Hutyra L. R. et al. & 2007 & $\begin{array}{l}\text { Journal of Geophysical Research: } \\
\text { Biogeosciences }\end{array}$ & 112 & 3 & G03008 & 10.1029/2006JG000365 \\
\hline 290 & $\begin{array}{l}\text { A regime shift from macrophyte to phytoplankton dominance enhances carbon burial in a shallow, eutrophic } \\
\text { lake }\end{array}$ & Brothers S. M. et al. & 2013 & Ecosphere & 4 & 11 & Art. 137 & 10.1890/ES13-00247.3 \\
\hline 291 & Habitat, not resource availability, limits consumer production in lake ecosystems & Craig N. et al. & 2015 & Limnology and Oceanography & 60 & 6 & 2079-2089 & 10.1002/Ino.10153 \\
\hline 292 & $\begin{array}{l}\text { From bacteria to piscivorous fish: Estimates of whole-lake and component-specific metabolism with an } \\
\text { ecosystem approach }\end{array}$ & Cremona F. et al. & 2014 & PLOS ONE & 9 & 7 & e101845 & 10.1371/journal.pone.0101845 \\
\hline 293 & Benthic community metabolism in deep and shallow Arctic lakes during 13 years of whole-lake fertilization & Daniels W. C. et al. & 2015 & Limnology and Oceanography & 60 & 5 & 1604-1618 & 3 10.1002/Ino.10120 \\
\hline 294 & $\begin{array}{l}\text { The importance of sedimenting organic matter, relative to oxygen and temperature, in structuring lake } \\
\text { profundal macroinvertebrate assemblages }\end{array}$ & Jyväsjärvi J. et al. & 2013 & Hydrobiologia & 709 & 1 & $55-72$ & 10.1007/s10750-012-1434-0 \\
\hline 295 & $\begin{array}{l}\text { Terrestrial organic matter support of lake food webs: Evidence from lake metabolism and stable hydrogen } \\
\text { isotopes of consumers }\end{array}$ & Karlsson J. et al. & 2012 & Limnology and Oceanography & 57 & 4 & 1042-1048 & 10.4319//o.2012.57.4.1042 \\
\hline 296 & Biogeochemical characteristics of settling particulate organic matter in Lake Superior: a seasonal comparison & Li H. and Minor E. C. & 2015 & Organic Geochemistry & 85 & & $76-88$ & 10.1016/j.orggeochem.2015.05.006 \\
\hline 297 & $\begin{array}{l}\text { Spatial and Temporal Variability in the Ecosystem Metabolism of a High-elevation Lake: Integrating Benthic } \\
\text { and Pelagic Habitats }\end{array}$ & Sadro S. et al. & 2011 & Ecosystems & 14 & 7 & $1123-1140$ & 10.1007/s10021-011-9471-5 \\
\hline 298 & $\begin{array}{l}\text { Accounting for littoral primary production by periphyton shifts a highly humic boreal lake towards net } \\
\text { autotrophy }\end{array}$ & Vesterinen J. et al. & 2016 & Freshwater Biology & 61 & 3 & $265-27$ & 10.1111/fwb.12700 \\
\hline 299 & Primary production of a seagrass bed on Kavaratti Atoll (Laccadives) & $\begin{array}{l}\text { Qasim S. Z. and Bhattathiri P. M. } \\
\text { A. }\end{array}$ & 1971 & Hydrobiologia & 38 & 1 & $29-38$ & 10.1007/BF00036790 \\
\hline 300 & Benthic metabolism across a gradient of anthropogenic impact in three shallow coastal lagoons in NW Florida & Stutes J. et al. & 2007 & Marine Ecology Progress Series & 348 & & $55-70$ & 10.3354/meps07036 \\
\hline 301 & $\begin{array}{l}\text { Organic Carbon Metabolism and Carbonate Dynamics in a Mediterranean Seagrass ( Posidonia oceanica ) } \\
\text { Meadow }\end{array}$ & Barrón C. et al. & 2006 & Estuaries and coasts & 29 & 3 & 417-426 & 10.1007/BF02784990 \\
\hline 302 & $\begin{array}{l}\text { Accumulation and transport of seagrass-derived organic matter in reef flat sediment of Green Island, Great } \\
\text { Barrier Reef }\end{array}$ & Miyajima T. et al. & 1998 & Marine Ecology Progress Series & 175 & & $251-259$ & 10.3354/meps175251 \\
\hline 303 & $\begin{array}{l}\text { The Detritic Compartment in a Posidonia oceanica Meadow : litter features, decomposition rates, and mineral } \\
\text { stocks }\end{array}$ & Romero J. et al. & 1992 & Marine Ecology & 13 & 1 & $69-83$ & 10.1111/j.1439-0485.1992.tb00341.x \\
\hline 304 & $\begin{array}{l}\text { Effects of suspended mussel culture on benthic-pelagic coupling in a coastal upwelling system (Ría de Vigo, } \\
\text { NW Iberian Peninsula) }\end{array}$ & Alonso-Pérez F. et al. & 2010 & $\begin{array}{l}\text { Journal of Experimental Marine Biology and } \\
\text { Ecology }\end{array}$ & 382 & 2 & 96-107 & 10.1016/j.jembe.2009.11.008 \\
\hline 305 & $\begin{array}{l}\text { Benthic fluxes, net ecosystem metabolism and seafood harvest: Completing the organic carbon balance in the } \\
\text { Ría de Vigo (NW Spain) }\end{array}$ & Alonso-Pérez F. et al. & 2015 & Estuarine, Coastal and Shelf Science & 163 & & 54-63 & 10.1016/j.ecss.2015.05.038 \\
\hline 306 & $\begin{array}{l}\text { A pelagic ecosystem model simulating production and sedimentation of biogenic particles: role of salps and } \\
\text { copepods }\end{array}$ & Andersen V. and Nival P. & 1988 & Marine Ecology Progress Series & 44 & 1 & $37-50$ & 10.3354/meps044037 \\
\hline 307 & A carbon budget for an oligotrophic clearwater lake in mid-Sweden & Andersson E. and Kumbald L. & 2006 & Aquatic Sciences & 68 & 1 & $52-64$ & $10.1007 / 500027-005-0807-0$ \\
\hline 308 & $\begin{array}{l}\text { Production and downward flux of zooplankton fecal pellets in the anticyclonic gyre off Shikoku, Japan } \\
\end{array}$ & Ayukai T. and Hattori H. & 1992 & Oceanologica Acta & 15 & 2 & $163-172$ & \\
\hline 309 & Phytoplankton productivity on the Canadian Shelf of the Beaufort Sea & Carmack E. C. et al. & 2004 & Marine Ecology Progress Series & 277 & $\begin{array}{c}\text { Augu } \\
\text { st }\end{array}$ & $37-50$ & 10.3354/meps277037 \\
\hline 310 & Spatial and temporal resolution of carbon fluxes in a shallow coastal ecosystem, the Kattegat & Carstensen J. et al. & 2003 & Marine Ecology Progress Series & 252 & & $35-50$ & 10.3354/meps252035 \\
\hline 311 & Carbon flows in a subtidal fine sand community from the western English Channel: a simulation analysis & Chardy P. and Dauvin J.-C. & 1992 & Marine Ecology Progress Series & 81 & 2 & $147-161$ & $10.3354 /$ mepso81147 \\
\hline 312 & Primary production and nutrients in a tropical macrotidal estuary, Darwin Harbour, Australia & Burford M. A. et al. & 2008 & Estuarine, Coastal and Shelf Science & 79 & 3 & $440-448$ & 10.1016/j.ecss.2008.04.018 \\
\hline 313 & $\begin{array}{l}\text { Metabolism of different benthic habitats and their contribution to the carbon budget of a shallow } \\
\text { oligotrophic sub-tropical coastal system (southern Moreton Bay, Australia) }\end{array}$ & Eyre B. D. et al. & 2011 & Biogeochemistry & 102 & 1 & 87-110 & 10.1007/s10533-010-9424-7 \\
\hline 314 & Nutrient cycling in the water column of a subtropical seagrass meadow & Ziegler S. and Benner R. & 1999 & Marine Ecology Progress Series & 188 & & $51-62$ & 10.3354/meps188051 \\
\hline 315 & $\begin{array}{l}\text { Carbon, nitrogen, and phosphorus budgets for a shallow subtropical coastal embayment (Moreton Bay, } \\
\text { Australia) }\end{array}$ & Eyre B. D. and Mckee L. J. & 2002 & Limnology and Oceanography & 47 & 4 & 1043-1055 & 10.4319//o.2002.47.4.1043 \\
\hline 316 & A partial budget of primary organic carbon flows in the littoral zone of a hardwater lake & Gessner M. O. et al & 1996 & Aquatic Botany & 55 & 2 & $93-105$ & 10.1016/S0304-3770(96)01064-9 \\
\hline 317 & $\begin{array}{l}\text { Primary production of aquatic macrophytes and their epiphytes in two shallow lakes (Peipsi and Võrtsjärv) in } \\
\text { Estonia }\end{array}$ & Nõges T. et al. & 2010 & Aquatic Ecology & 44 & 1 & 83-92 & 10.1007/s10452-009-9249-4 \\
\hline 318 & Benthic and planktonic primary production along a nutrient gradient in Green Bay, Lake Michigan, USA & Althouse B. et al. & 2014 & Freshwater Science & 33 & 2 & $487-498$ & $10.1086 / 676314$ \\
\hline 319 & $\begin{array}{l}\text { Carbon, nitrogen, oxygen and sulfide budgets in the Black Sea: A biogeochemical model of the whole water } \\
\text { column coupling the oxic and anoxic parts }\end{array}$ & Grégoire M. and Soetaert K. & 2010 & Ecological Modelling & 221 & & 2287-2301 & 10.1016/j.ecolmodel.2010.06.007 \\
\hline 320 & The composition of particulate organic matter in the euphotic zone of Lake Superior & Halfon E. & 1984 & Journal of Great Lakes Research & 10 & 3 & $299-306$ & $10.1016 / 50380-1330(84) 71843-0$ \\
\hline
\end{tabular}




\begin{tabular}{|c|c|c|c|c|c|c|c|c|}
\hline 321 & $\begin{array}{l}\text { Carbon and nitrogen isotopic compositions of particulate organic matter in four large river systems across the } \\
\text { United States }\end{array}$ & Kendall C. et al. & 2001 & Hydrological Processes & 15 & 7 & 1301-1346 & 10.1002/hyp.216 \\
\hline 322 & $\begin{array}{l}\text { Seasonal shifts between autochthonous and allochthonous carbon contributions to zooplankton diets in a } \\
\text { subarctic lake }\end{array}$ & Rautio M. et al. & 2011 & Limnology and Oceanography & 56 & 4 & 1513-1524 & 10.4319/lo.2011.56.4.1513 \\
\hline 323 & $\begin{array}{l}\text { Nutrient uptake and release during growth and decomposition of eelgrass, Zoostera Marina L., and its effects } \\
\text { on the nutrient dynamics of Lake Grevelingen }\end{array}$ & Pellikan G. C. and Nienhuis P. H. & 1988 & Aquatic Botany & 30 & 3 & $189-214$ & $\underline{10.1016 / 0304-3770(88) 90051-4}$ \\
\hline 324 & Metabolic imbalance in coastal vegetated (Posidonia oceanica) and unvegetated benthic ecosystems & Apostolaki E. et al. & 2010 & Ecosystems & 13 & 3 & $459-471$ & $10.1007 /$ /s10021-010-9330-9 \\
\hline 325 & Detrital stocks and dynamics of the seagrass Posidonia oceanica (L.) Delile in the Spanish Mediterranean & Cebrian J. and Duarte C. M. & 2001 & Aquatic Botany & 70 & & 295-309 & $10.1016 /$ S0304-3770(01)00154-1 \\
\hline 326 & $\begin{array}{l}\text { Biomass dynamics of seagrasses and the role of mangrove and seagrass vegetation as different nutrient } \\
\text { sources for an intertidal ecosystem }\end{array}$ & De Boer W. F. & 2000 & Aquatic Botany & 66 & & 225-239 & 10.1016/S0304-3770(99)00072-8 \\
\hline 327 & Bioavailability of dissolved organic carbon linked with the regional carbon cycle in the East China Sea & Gan S. et al. & 2016 & $\begin{array}{l}\text { Deep Sea Research Part II: Topical Studies in } \\
\text { Oceanography }\end{array}$ & 124 & & $19-28$ & 10.1016/j.dsr2.2015.06.024 \\
\hline 328 & $\begin{array}{l}\text { Carbon fluxes within the pelagic food web in the coastal area off Antofagasta }\left(23^{\circ} \mathrm{S}\right) \text {, Chile: The significance of } \\
\text { the microbial versus classical food webs }\end{array}$ & Pavés H. J. and González H. E. & 2008 & Ecological Modelling & 211 & 3-4 & 218-232 & 10.1016/j.ecolmodel.2007.10.004 \\
\hline 329 & $\begin{array}{l}\text { Primary production and community respiration in the Humboldt Current System off Chile and associated } \\
\text { oceanic areas }\end{array}$ & Danieri G. et al. & 2000 & Marine Ecology Progress Series & 197 & & 41-49 & 10.3354/meps197041 \\
\hline 330 & Integrated assessment of the carbon budget in the southeastern Bering Sea & Cross J. N. et al. & 2014 & $\begin{array}{l}\text { Deep Sea Research Part II: Topical Studies in } \\
\text { Oceanography }\end{array}$ & 109 & & $112-124$ & 10.1016/j.dsr2.2014.03.003 \\
\hline 331 & $\begin{array}{l}\text { Benthic trophic dynamics in California coastal|rbasin and continental slope communities inferred using } \\
\text { inverse analysis }\end{array}$ & Eldridge P. M. and Jackson G. A. & 1993 & Marine Ecology Progress Series & 99 & & $115-135$ & 10.3354/meps099115 \\
\hline 332 & $\begin{array}{l}\text { Seasonal changes of the quantitative importance of protozoans in a large lake: An ecosystem approach using } \\
\text { mass-balanced carbon flow diagrams. }\end{array}$ & Gaedke U. and Straile D. & 1994 & Marine Microbial Food Webs & 8 & & $163-188$ & $\begin{array}{l}\text { http://nbn- } \\
\text { resolving.de/urn:nbn:de:bsz:352-opus- } \\
39463\end{array}$ \\
\hline 333 & Benthic 02 exchange across hard-bottom substrates quantified by eddy correlation in a sub-Arctic fjord & Glud R. N. et al. & 2010 & Marine Ecology Progress Series & 417 & & $1-12$ & $10.3354 /$ meps08795 \\
\hline 334 & $\begin{array}{l}\text { Seasonal plankton variability in Chilean Patagonia fjords: Carbon flow through the pelagic food web of Aysen } \\
\text { Fjord and plankton dynamics in the Moraleda Channel basin }\end{array}$ & González H. E. et al. & 2011 & Continental Shelf Research & 31 & & $225-243$ & 10.1016/j.csr.2010.08.010 \\
\hline 335 & Dynamics of the pelagic food web in St. Georges Bay, southern Gulf of St. Lawrence & Hargrave B. T. et al. & 1985 & Marine Ecology Progress Series & 20 & & $221-240$ & $10.3354 /$ meps020221 \\
\hline 336 & Carbon metabolism in a humic lake: Pool sizes and cycling through zooplankton & Hessen D. O. et al. & 1990 & Limnology and Oceanography & 35 & 1 & $84-99$ & $10.4319 / / 0.1990 .35 .1 .0084$ \\
\hline 337 & A carbon flow model and network analysis of the northern Benguela upwelling system, Namibia & Heymans J. J. and Baird D. & 2000 & Ecological Modelling & 126 & 1 & $9-32$ & 10.1016/50304-3800(99)00192-1 \\
\hline 338 & Carbon budget of the planktonic food web in an atoll lagoon (Takapoto, French Polynesia) & Sakka A. et al. & 2002 & Journal of Plankton Research & 24 & 4 & $301-320$ & 10.1093/plankt/24.4.301 \\
\hline 339 & $\begin{array}{l}\text { A comparison of selected ecosystem attributes of three South African estuaries with different freshwater } \\
\text { inflow regimes, using network analysis }\end{array}$ & Scharler U. M. and Baird D. & 2005 & Journal of Marine Systems & 56 & 3-4 & 283-308 & 10.1016/j.jmarsys.2004.12.003 \\
\hline 340 & Benthic exchange and biogeochemical cycling in permeable sediments. & Huettel M. et al. & 2014 & Annual Review of Marine Science & 6 & & $23-51$ & 10.1146/annurev-marine-051413-012706 \\
\hline 341 & $\begin{array}{l}\text { Seasonal variability of primary production in a fjord ecosystem of the Chilean Patagonia: Implications for the } \\
\text { transfer of carbon within pelagic food webs }\end{array}$ & Montero P. et al. & 2011 & Continental Shelf Research & 31 & $3-4$ & $202-215$ & 10.1016/j.csr.2010.09.003 \\
\hline 342 & $\begin{array}{l}\text { Measurements of seasonal rates and annual budgets of organic carbon fluxes in an Antarctic coastal } \\
\text { environment at Signy Island, South Orkney Islands, suggest a broad balance between production and } \\
\text { decomposition }\end{array}$ & Nedwell D. B. et al. & 1993 & Applied and Environmental Microbiology & 59 & 12 & 3989-3995 & \\
\hline 343 & Benthic primary production budget of a Caribbean reef lagoon (Puerto Morelos, Mexico) & Naumann M. S. et al. & 2013 & PLOS ONE & 8 & 12 & e82923 & 10.1371/journal.pone.0082923 \\
\hline 344 & Budget of coral-derived organic carbon in a fringing coral reef of the Gulf of Aqaba, Red Sea & Naumann M. S. et al. & 2012 & Journal of Marine Systems & 105-108 & & $20-29$ & 10.1016/j.jmarsys.2012.05.007 \\
\hline 345 & $\begin{array}{l}\text { Recycling of organic matter in antarctic sediments: a transect through the polar front in the southern ocean } \\
\text { (Indian Sector) }\end{array}$ & Rabouille C. et al. & 1998 & Limnology and Oceanography & 43 & 3 & $420-432$ & 10.4319/10.1998.43.3.0420 \\
\hline 346 & Dynamics of carbon and nitrogen during the decomposition of detritus derived from estuarine macrophytes & Rice D. L. and Tenore K. R. & 1981 & Estuarine, Coastal and Shelf Science & 13 & 6 & $681-690$ & $10.1016 / 50302-3524(81) 80049-7$ \\
\hline 347 & $\begin{array}{l}\text { Carbon cycling in mesohaline Chesapeake Bay sediments .2. Kinetics of particulate and dissolved organic } \\
\text { carbon turnover }\end{array}$ & Roden E. E. and Tuttle J. H. & 1996 & Journal of Marine Research & 54 & 2 & $343-383$ & $10.1357 / 0022240963213349$ \\
\hline 348 & Analysis of the trophic network of a macrotidal ecosystem: The Bay of Somme (Eastern Channel) & Rybarczyk H. et al. & 2003 & Estuarine, Coastal and Shelf Science & 58 & 3 & 405-421 & $10.1016 / 50272-7714(02) 00294-9$ \\
\hline 349 & Comparative ecosystem trophic structure of three US mid- Atlantic estuaries & $\begin{array}{l}\text { Monaco M. E. and Ulanowicz R. } \\
\text { E. }\end{array}$ & 1997 & Marine Ecology Progress Series & 161 & & 239-254 & 10.3354/meps161239 \\
\hline 350 & Carbon flows through a benthic food web: Integrating biomass, isotope and tracer data & Van Oevelen D. & 2006 & Journal of Marine Research & 64 & 3 & 453-482 & $10.1357 / 002224006778189581$ \\
\hline 351 & $\begin{array}{l}\text { Export of biogenic carbon and structure and dynamics of the pelagic food web in the Gulf of St. Lawrence Part } \\
\text { 2. Inverse analysis }\end{array}$ & Vézina A. F. et al. & 2000 & $\begin{array}{l}\text { Deep-Sea Research Part II: Topical Studies in } \\
\text { Oceanography }\end{array}$ & 47 & $3-4$ & 609-635 & 10.1016/50967-0645(99)00120-4 \\
\hline 352 & $\begin{array}{l}\text { Quantification of functional soil organic carbon pools for major soil units and land uses in southeast Germany } \\
\text { (Bavaria) }\end{array}$ & Wiesmeier M. et al. & 2014 & Agriculture, Ecosystems and Environment & 185 & & $208-220$ & 10.1016/j.agee.2013.12.028 \\
\hline 353 & Litter processing along a stream gradient: the role of invertebrates and decomposers & Graça M. A. S. et a.I & 2001 & J. of the North Am. Benth. Soc. & 20 & 3 & $408-420$ & $10.2307 / 1468038$ \\
\hline
\end{tabular}




\begin{tabular}{|c|c|c|c|c|c|c|c|c|}
\hline 354 & $\begin{array}{l}\text { Ecosystem linkages between southern Appalachian headwater streams and their banks: Leaf litter breakdown } \\
\text { and invertebrate assemblages }\end{array}$ & $\begin{array}{l}\text { Hutchens J. J. Jr. and Wallace J. } \\
\text { B. }\end{array}$ & 2002 & Ecosystems & 5 & 1 & 80-91 & $10.1007 / \mathrm{s} 10021-001-0057-5$ \\
\hline 355 & Latitudinal patterns in leaf litter breakdown: Is temperature really important? & Irons III J. G. et al. & 1994 & Freshwater Biology & 32 & 2 & 401-411 & 10.1111/j.1365-2427.1994.tb01135.x \\
\hline 356 & Forest floor decomposition following hurricane litter inputs in several Puerto Rican forests & Ostertag R. et al. & 2003 & Ecosystems & 6 & 3 & $261-273$ & 10.1007/s10021-002-0203-8 \\
\hline 357 & Leaf litter breakdown rates in boreal streams: Does shredder species richness matter? & Jonsson M. et al. & 2001 & Freshwater Biology & 46 & 2 & $161-171$ & $10.1046 / j .1365-2427.2001 .00655 . x$ \\
\hline 358 & Leaf decomposition in two semi-evergreen tropical forests: Influence of litter quality & Loranger G. et al. & 2002 & Biology and Fertility of Soils & 35 & 4 & $247-252$ & $10.1007 / 500374-002-0467-3$ \\
\hline 359 & Macroconsumer effects on insect detritivores and detritus processing in a tropical stream & Rosemond A. D. et al. & 1998 & Freshwater Biology & 39 & 3 & $515-523$ & 10.1046/j.1365-2427.1998.00301.x \\
\hline 360 & Leaf litter processing rates in a Kenyan highland stream, the Njoro River & Dobson M. et al. & 2004 & Hydrobiologia & 519 & & $207-210$ & 10.1023/B:HYDR.0000026592.50734.ea \\
\hline 361 & Effects of nutrient enrichment and leaf quality on the breakdown of leaves in a hardwater stream & Royer T. V. and Minshall W. G. & 2001 & Freshwater Biology & 46 & 5 & $603-610$ & $10.1046 / \mathrm{j} .1365-2427.2001 .00694 . x$ \\
\hline 362 & $\begin{array}{l}\text { Over-winter decomposition and associated macroinvertebrate communities of three deciduous leaf species in } \\
\text { forest streams on the Canadian boreal shield }\end{array}$ & Muto E. A. et al. & 2011 & Hydrobiologia & 658 & 1 & $111-126$ & 10.1007/s10750-010-0455-9 \\
\hline 363 & Effects of nutrient enrichment on boreal streams: Invertebrates, fungi and leaf-litter breakdown & Bergfur J. et al. & 2007 & Freshwater Biology & 52 & 8 & $1618-1633$ & $10.1111 / \mathrm{j} .1365-2427.2007 .01770 . \mathrm{x}$ \\
\hline 364 & $\begin{array}{l}\text { The hippopotamus conveyor belt : vectors of carbon and nutrients from terrestrial grasslands to aquatic } \\
\text { systems in sub-Saharan Africa }\end{array}$ & Subaluski A. L. et al. & 2015 & Freshwater Biology & 60 & 3 & $512-525$ & 10.1111/fwb.12474 \\
\hline 365 & Shifts in reciprocal river-riparian arthropod fluxes along an urban-rural landscape gradient & Kautza A. and Sullivan S. M. P. & 2015 & Freshwater Biology & 60 & 10 & $2156-2168$ & 10.1111/fwb.12642 \\
\hline 366 & Leaf litter quality affects aquatic insect emergence: Contrasting patterns from two foundation trees & Compson Z. G. et al. & 2013 & Oecologia & 173 & 2 & $507-519$ & 10.1007/s00442-013-2643-6 \\
\hline 367 & Roe deer may markedly alter forest nitrogen and phosphorus budgets across Europe & Abbas F. et al. & 2012 & Oikos & 121 & 8 & $1271-1278$ & $10.1111 / \mathrm{j} .1600-0706.2011 .20103 . x$ \\
\hline 368 & Flux of aquatic insect productivity to land: comparison of lentic and lotic ecosystems & $\begin{array}{l}\text { Gratton C. and Vander Zanden } \\
\text { J. M. }\end{array}$ & 2009 & Ecology & 90 & 10 & $2689-2699$ & $10.1890 / 08-1546.1$ \\
\hline 369 & $\begin{array}{l}\text { Forest-stream linkages: Effects of terrestrial invertebrate input and light on diet and growth of brown trout } \\
\text { (salmo trutta) in a boreal forest stream }\end{array}$ & Eros T. et al. & 2012 & PLOS ONE & 7 & 5 & e36462 & 10.1371/journal.pone.0036462 \\
\hline 370 & $\begin{array}{l}\text { Woody debris and terrestrial invertebrates - effects on prey resources for brown trout (Salmo trutta) in a } \\
\text { boreal stream }\end{array}$ & Gustafsson P. et al. & 2014 & Environmental Biology of Fishes & 97 & 5 & $529-542$ & $10.1007 / \mathrm{s} 10641-014-0250-y$ \\
\hline 371 & Marine Macrophyte Wrack Inputs and Dissolved Nutrients in Beach Sands & Dugan J. E. et al. & 2011 & Estuaries and coasts & 34 & 4 & $839-850$ & $10.1007 /$ s12237-011-9375-9 \\
\hline 372 & Ecosystem connectivity and trophic subsidies of sandy beaches & Liebowitz D. M. et al. & 2016 & Ecosphere & 7 & 10 & Art.e01503 & $10.1002 /$ ecs2.1503 \\
\hline 373 & $\begin{array}{l}\text { Changes in biomass and botancial compsition of beach-cast seaweeds in a dirturbed coastal area from } \\
\text { Argentine Patagonia }\end{array}$ & Piriz M. L. et al. & 2003 & Journal of applied Phycology & 15 & & $67-74$ & 10.1023/A:1022959005072 \\
\hline 374 & $\begin{array}{l}\text { Cross-ecosystem fluxes: Export of polyunsaturated fatty acids from aquatic to terrestrial ecosystems via } \\
\text { emerging insects }\end{array}$ & Martin-Creuzburg D. et al. & 2017 & Science of the Total Environment & 577 & & $174-182$ & 10.1016/j.scitotenv.2016.10.156 \\
\hline 375 & $\begin{array}{l}\text { Deposition and decomposition of periodical cicadas (Homoptera: Cicadidae: Magicicauda) in woodland } \\
\text { aquatic ecosystems }\end{array}$ & Pray C. L. et al. & 2009 & J. of the North Am. Benth. Soc. & 28 & 1 & $181-195$ & $10.1899 / 08-038.1$ \\
\hline 376 & Patterns of herbivory and decomposition in aquatic and terrestrial ecosystems & Cebrian J. and Lartigue J. & 2004 & Ecological Monographs & 74 & 2 & $237-259$ & $10.1890 / 03-4019$ \\
\hline 377 & $\begin{array}{l}\text { Above-Ground Crop Mass and Species Richness of the Principal Types of Herbaceous Rich- Fen Vegetation of } \\
\text { Lowland England and Wales }\end{array}$ & Wheeler B. D. and Shaw S. C. & 1991 & Journal of Ecology & 79 & 2 & 285-301 & $10.2307 / 2260713$ \\
\hline 378 & Energy and Nutrient Dynamics of Spider and Orthopteran Populations in a Grassland Ecosystem & Van Hook R. I. Jr. & 1971 & Ecological Monographs & 41 & 1 & $1-26$ & $10.2307 / 1942433$ \\
\hline 379 & $\begin{array}{l}\text { Cycling of organic carbon in the photic zone of a eutrophic lake with special reference to the heterotrophic } \\
\text { bacteria }\end{array}$ & Vadstein O. et al. & 1989 & Limnology and Oceanography & 34 & 5 & $840-855$ & 10.4319/10.1989.34.5.0840 \\
\hline 380 & $\begin{array}{l}\text { Medium-term effects of corn biochar addition on soil biota activities and functions in a temperate soil } \\
\text { cropped to corn }\end{array}$ & Domene $X$. et al. & 2014 & Soil Biology and Biochemistry & 72 & & $152-162$ & 10.1016/j.soilbio.2014.01.035 \\
\hline 381 & Management of small grain residues to retain legume-derived nitrogen in corn cropping systems & Starovoytov A. et al. & 2010 & Agronomy Journal & 102 & 3 & $895-903$ & 10.2134/agronj2009.0402 \\
\hline 382 & Coupling between macroalgal inputs and nutrients outcrop in exposed sandy beaches & Barreiro F. et al. & 2013 & Hydrobiologia & 700 & 1 & $73-84$ & 10.1007/s10750-012-1220-z \\
\hline 383 & $\begin{array}{l}\text { Fluxes of biomass and essential polyunsaturated fatty acids from water to land via chironomid emergence } \\
\text { from a mountain lake }\end{array}$ & Borisova E. V. et al. & 2016 & Contemporary Problems of Ecology & 9 & 4 & $446-457$ & 10.1134/S199542551604003X \\
\hline 384 & Upper Ocean Carbon Export and the Biological Pump & Ducklow H. W. et al. & 2001 & Oceanography & 14 & 4 & $50-58$ & 10.5670/oceanog.2001.06 \\
\hline 385 & Importance of copepod carcasses versus faecal pellets in the upper water column of an oligotrophic area & Frangoulis C. et al. & 2011 & Estuarine, Coastal and Shelf Science & 92 & 3 & $456-463$ & 10.1016/j.ecss.2011.02.005 \\
\hline 386 & Magnitude and fate of salmon-derived nutrients and energy in a coastal stream ecosystem & Gende S. M. et al. & 2004 & Journal of Freshwater Ecology & 19 & 1 & $149-160$ & $10.1080 / 02705060.2004 .9664522$ \\
\hline 387 & Carbon and nitrogen transfer from a desert stream to riparian predators & Sanzone D. M. et al. & 2003 & Oecologia & 134 & 2 & $238-250$ & 10.1007/s00442-002-1113-3 \\
\hline 388 & Sources of carbon fuelling production in an arid floodplain river & Burford M. A. et al. & 2008 & Marine and Freshwater research & 59 & 3 & $224-234$ & 10.1071/MF07159 \\
\hline 389 & $\begin{array}{l}\text { Effects of the abundance of spawning sockeye salmon ( Oncorhynchus nerka ) on nutrients and algal biomass } \\
\text { in forested streams }\end{array}$ & Johnston N. T. et al. & 2004 & Can. J. of Fish. and Aq. Sci. & 61 & $\begin{array}{c}\text { Augu } \\
\text { st }\end{array}$ & 384-403 & 10.1139/F03-172 \\
\hline 390 & Density-dependent predation by brown bears ( Ursus arctos ) on sockeye salmon ( Oncorhynchus nerka ) & Quinn T.P. et al. & 2003 & Can. J. of Fish. and Aq. Sci. & 60 & & $553-562$ & $10.1139 / \mathrm{F} 03-045$ \\
\hline
\end{tabular}




\begin{tabular}{|c|c|c|c|c|c|c|c|c|}
\hline 391 & Large annual net ecosystem $\mathrm{CO} 2$ uptake of a Mojave Desert ecosystem & Wohlfahrt G. et al. & 2008 & Global Change Biology & 14 & 7 & 1475-1487 & $10.1111 / j .1365-2486.2008 .01593 . x$ \\
\hline 392 & Coarse particulate detritus dynamics in small, montane streams of southwestern British Columbia & Richardson J. S. & 1992 & Can. J. of Fish. and Aq. Sci. & 49 & & $337-346$ & $10.1139 / \mathrm{fg2}-038$ \\
\hline 393 & Carbon and nutrient deposition in a Mediterranean seagrass ( Posidonia oceanica ) meadow & Gacia E. et al. & 2002 & Limnology and Oceanography & 47 & 1 & $23-32$ & 10.4319//o.2002.47.1.0023 \\
\hline 394 & Carbon and water fluxes in an exotic buffelgrass savanna & Hinojo-Hinojo C. et al. & 2016 & Rangeland Ecology and Management & 69 & 5 & $334-341$ & 10.1016/j.rama.2016.04.002 \\
\hline 395 & Seasonal and Annual Variations of Carbon Dioxide Fluxes in Desert Ecosystem & Liu R. et al. & 2011 & Journal of Desert Research & 31 & & $104-108$ & $\begin{array}{l}\text { http://en.cnki.com.cn/Article_en/CJFDTOT } \\
\text { AL-ZGSS201101018.htm }\end{array}$ \\
\hline 396 & $\begin{array}{l}\text { Diurnal, seasonal and annual variation in the net ecosystem CO2 exchange of a desert shrub community } \\
\text { (Sarcocaulescent) in Baja California, Mexico }\end{array}$ & Hastings S. J. et al. & 2005 & Global Change Biology & 11 & 6 & 927-939 & 10.1111/j.1365-2486.2005.00951.x \\
\hline 397 & Carbon dioxide fluxes in a semiarid environment with high carbonate soils & Emmerich W. E. & 2003 & Agricultural and Forest Meteorology & 116 & $1-2$ & $91-102$ & $10.1016 / 50168-1923(02) 00231-9$ \\
\hline 398 & Carbon sequestration in arid-land forest & Grünzweig J. M. et al. & 2003 & Global Change Biology & 9 & & 791-799 & 10.1046/j.1365-2486.2003.00612.x \\
\hline 399 & $\begin{array}{l}\text { Response of vegetation and soil carbon and nitrogen storage to grazing intensity in semi-arid grasslands in } \\
\text { the agro-pastoral zone of northern china }\end{array}$ & Xu M.Y. et al. & 2014 & PLOS One & 9 & 5 & e96604 & 10.1371/journal.pone.0096604 \\
\hline 400 & Seasonal variation in carbon dioxide exchange over a Mediterranean annual grassland in California & Xu L. and Baldocchi D. D. & 2004 & Agricultural and Forest Meteorology & 123 & $1-2$ & $79-96$ & 10.1016/j.agrformet.2003.10.004 \\
\hline 401 & $\begin{array}{l}\text { Relationship between sediment organic matter, bacteria composition, and the ecosystem metabolism of } \\
\text { alpine streams }\end{array}$ & Logue J. B. et al. & 2004 & Limnology and Oceanography & 49 & 6 & 2001-2010 & $10.4319 / 10.2004 .49 .6 .2001$ \\
\hline 402 & $\begin{array}{l}\text { Diurnal and seasonal variations in carbon dioxide exchange in ecosystems in the Zhangye oasis Area, } \\
\text { northwest China }\end{array}$ & Zhang L. et al. & 2015 & PLOS One & 10 & 3 & $1-16$ & 10.1371/journal.pone. 0120660 \\
\hline 403 & $\begin{array}{l}\text { Responses of gross primary productivity to different sizes of precipitation events in a temperate grassland } \\
\text { ecosystem in Inner Mongolia, China }\end{array}$ & Guo Q. et al. & 2016 & Journal of Arid Land & 8 & 1 & $36-46$ & $10.1007 / 540333-015-0136-7$ \\
\hline 404 & $\begin{array}{l}\text { Patterns of } \mathrm{CO} 2 \text { exchange and productivity of the herbaceous vegetation and trees in a humid savanna in } \\
\text { western Kenya }\end{array}$ & Otieno D. et al. & 2015 & Plant Ecology & 216 & 10 & 1441-1456 & $10.1007 / 511258-015-0523-3$ \\
\hline 405 & $\begin{array}{l}\text { Elevated carbon dioxide alters impacts of precipitation pulses on ecosystem photosynthesis and respiration in } \\
\text { a semi-arid grassland }\end{array}$ & Bachman S. et al. & 2010 & Oecologia & 162 & 3 & 791-802 & $10.1007 / 500442-009-1511-x$ \\
\hline 406 & $\begin{array}{l}\text { Grazing exclusion significantly improves grassland ecosystem C and N pools in a desert steppe of Northwest } \\
\text { China }\end{array}$ & Wang K. et al. & 2016 & Catena & 137 & & $441-448$ & 10.1016/j.catena.2015.10.018 \\
\hline 407 & $\begin{array}{l}\text { Dynamics of soil carbon following destruction of tropical rainforest and the subsequent establishment of } \\
\text { Imperata grassland in Indonesian Borneo using stable carbon isotopes }\end{array}$ & Yonekura Y. et al. & 2012 & Global Change Biology & 18 & & 2606-2616 & $10.1111 / \mathrm{j} .1365-2486.2012 .02722 . x$ \\
\hline 408 & $\begin{array}{l}\text { Eddy covariance and biometric measurements show that a savanna ecosystem in Southwest China is a carbon } \\
\text { sink }\end{array}$ & Fei X. et al. & 2017 & Scientific Reports & 7 & & 41025 & 10.1038/srep41025 \\
\hline 409 & $\begin{array}{l}\text { Soil carbon balance in a tropical grassland: Estimation of soil respiration and its partitioning using a semi- } \\
\text { empirical model }\end{array}$ & Caquet B. et al. & 2012 & Agricultural and Forest Meteorology & $158-159$ & & 71-79 & 10.1016/j.agrformet.2012.02.008 \\
\hline 410 & Seasonal dynamics in carbon dioxide fluxes of the herbaceous layer of a moist Kenyan savannah & K'Otuto G. O. et al. & 2014 & Applied Ecology and Environmental Research & 12 & 1 & $63-82$ & ISSN 17850037 (Online) \\
\hline 411 & Carbon fluxes from an urban tropical grassland & Ng B.J. L. et al. & 2015 & Environmental Pollution & 203 & & $227-234$ & 10.1016/j.envpol.2014.06.009 \\
\hline 412 & Carbon dynamics in an Imperata grassland in Northeast India & Thokchom A. and Yadava P. S. & 2016 & Tropical Grasslands - Forrajes Tropicales & 4 & 1 & $19-28$ & $10.17138 /$ TGFT(4)19-28 \\
\hline 413 & Regional patterns in carbon cycling across the Great Plains of North America & McCulley R. L. et al. & 2005 & Ecosystems & 8 & 1 & $106-121$ & $10.1007 / \mathrm{s} 10021-004-0117-8$ \\
\hline 414 & Impact of Land-Use Change on Carbon Stocks in Meadow Steppe of Northeast China & Lian P. et al. & 2011 & Applied Mechanics and Materials & 108 & & $262-268$ & 10.4028/www.scientific.net/AMM.108.262 \\
\hline 415 & Formation of soil organic matter via biochemical and physical pathways of litter mass loss & Cotrufo M. F. et al. & 2015 & Nature Geosciences & 8 & 10 & $776-779$ & $10.1038 /$ ngeo2520 \\
\hline 416 & $\begin{array}{l}\text { Millipedes and earthworms increase the decomposition rate of } 15 \mathrm{~N} \text {-labelled winter rape litter in an arable } \\
\text { field }\end{array}$ & Martens H. et al. & 2001 & Isotopes in environmental and health studies & 37 & 1 & $43-51$ & 10.1080/10256010108033280 \\
\hline 417 & $\begin{array}{l}\text { Dynamics in the trophic structure of the macroinvertebrate community in a Mediterranean, temporary } \\
\text { stream }\end{array}$ & Álvarez M. and Pardo I. & 2009 & Aquatic Sciences & 71 & 2 & $202-213$ & 10.1007/s00027-009-9160-z \\
\hline 418 & Carbon budget of leaves of the tropical intertidal seagrass Thalassia hemprichii & Chiu S.-H. et al. & 2013 & Estuarine, Coastal and Shelf Science & 125 & & $27-35$ & 10.1016/j.ecss.2013.03.026 \\
\hline 419 & $\begin{array}{l}\text { Response of early Ruppia cirrhosa litter breakdown to nutrient addition in a coastal lagoon affected by } \\
\text { agricultural runoff }\end{array}$ & Menéndez M. & 2009 & Estuarine, Coastal and Shelf Science & 82 & 4 & $608-614$ & 10.1016/j.ecss.2009.02.029 \\
\hline 420 & Carbon budgets of multispecies seagrass beds at Dongsha Island in the South China Sea. & Huang Y.-H.et al & 2015 & Marine Environmental Research & 106 & & $92-102$ & 10.1016/j.marenvres.2015.03.004 \\
\hline 421 & A wildfire event influences ecosystem carbon fluxes but not soil respiration in a semi-arid woodland & Sun $Q$. et al. & 2016 & Agricultural and Forest Meteorology & 226-227 & & $57-66$ & 10.1016/j.agrformet.2016.05.019 \\
\hline 422 & C stocks in forest floor and mineral soil of two mediterranean beech forests & De Marco A. et al. & 2016 & Forests & 7 & & 181 & $10.3390 / f 7080181$ \\
\hline 423 & $\begin{array}{l}\text { Enhanced summer warming reduces fungal decomposer diversity and litter mass loss more strongly in dry } \\
\text { than in wet tundra }\end{array}$ & Christiansen C. T. et al. & 2017 & Global Change Biology & 23 & & $406-420$ & 10.1111/gcb.13362 \\
\hline 424 & $\begin{array}{l}\text { Plant production and nitrogen accumulation above- and belowground in low and tall birch tundra } \\
\text { communities: the influence of snow and litter }\end{array}$ & $\begin{array}{l}\text { Vankoughnett M. R. and Grogan } \\
\text { P. }\end{array}$ & 2016 & Plant and Soil & 408 & $1-2$ & $195-210$ & 10.1007/s11104-016-2921-2 \\
\hline
\end{tabular}




\begin{tabular}{|c|c|c|c|c|c|c|c|c|}
\hline 425 & $\begin{array}{l}\text { The effects of snowpack properties and plant strategies on litter decomposition during winter in subalpine } \\
\text { meadows }\end{array}$ & Saccone P. et al. & 2013 & Plant and Soil & 363 & $1-2$ & $215-229$ & $10.1007 /$ s11104-012-1307-3 \\
\hline 426 & An integrated assessment of ecosystem carbon pools and fluxes across an oceanic alpine toposequence & Britton A. B. et al. & 2011 & Plant and Soil & 345 & 1 & $287-302$ & 10.1007/s11104-011-0781-3 \\
\hline 427 & Carbon dioxide fluxes over a temperate meadow in eastern Inner Mongolia, China & Jing $Y$. et al. & 2014 & Environmental Earth Sciences & 72 & 11 & 4401-4411 & $10.1007 /$ s12665-014-3341-3 \\
\hline 428 & $\begin{array}{l}\text { Effect of manure application on seasonal carbon fluxes in a temperate managed grassland in Southern } \\
\text { Hokkaido, Japan }\end{array}$ & Shimizu M. et al. & 2015 & Catena & 133 & & 474-485 & 10.1016/j.catena.2015.05.011 \\
\hline 429 & $\begin{array}{l}\text { Long-term grazing exclusion effects on vegetation characteristics, soil properties and bacterial communities in } \\
\text { the semi-arid grasslands of China }\end{array}$ & Cheng J. et al. & 2016 & Ecological Engineering & 97 & & $170-178$ & 10.1016/j.ecoleng.2016.09.003 \\
\hline 430 & Carbon and nutrient exchange of mangrove forests with the coastal ocean & Adame M. F. and Lovelock C. E. & 2011 & Hydrobiologia & 663 & 1 & $23-50$ & $10.1007 / \mathrm{s} 10750-010-0554-7$ \\
\hline 431 & $\begin{array}{l}\text { Short-term contributions of cover crop surface residue return to soil carbon and nitrogen contents in } \\
\text { temperate Australia }\end{array}$ & Zhou X. et al. & 2016 & $\begin{array}{l}\text { Environmental Science and Pollution } \\
\text { Research }\end{array}$ & 23 & 22 & $\begin{array}{r}23175- \\
23183 \\
\end{array}$ & $10.1007 / 511356-016-7549-5$ \\
\hline 432 & Do sheep grazing patterns affect ecosystem functioning in steppe grassland ecosystems in Inner Mongolia? & Ren H. et al. & 2015 & Agriculture, Ecosystems and Environment & 213 & & $1-10$ & 10.1016/j.agee.2015.07.015 \\
\hline 433 & Photodegradation of plant litter in the Sonoran Desert varies by litter type and age & Day T. A. et al. & 2015 & Soil Biology and Biochemistry & 89 & & $109-122$ & 10.1016/j.soilbio.2015.06.029 \\
\hline 434 & $\begin{array}{l}\text { Vegetation and soil property response of short-time fencing in temperate desert of the Hexi Corridor, } \\
\text { northwestern China }\end{array}$ & Zhang Y. and Zhao $\mathrm{W}$. & 2015 & Catena & 133 & & $43-51$ & 10.1016/j.catena.2015.04.019 \\
\hline 435 & $\begin{array}{l}\text { Agricultural Activities of a Meadow Eliminated Plant Litter from the Periphery of a Farmland in Inner } \\
\text { Mongolia, China }\end{array}$ & Kawada K. et al. & 2015 & PLOS One & 10 & 8 & $1-13$ & 10.1371/journal.pone.0135077 \\
\hline 436 & Effects of grassland conversion from cropland on soil respiration on the semi-arid loess plateau, China & Wang D. et al. & 2015 & Clean - Soil, Air, Water & 43 & 7 & 1052-1057 & $10.1002 /$ clen.201300971 \\
\hline 437 & $\begin{array}{l}\text { Are there any trade-offs between forage provision and the ecosystem service of } \mathrm{C} \text { and } \mathrm{N} \text { storage in arid } \\
\text { rangelands? }\end{array}$ & Oñatibia G. R. et al. & 2015 & Ecological Engineering & 77 & & $26-32$ & 10.1016/j.ecoleng.2015.01.009 \\
\hline 438 & The role of shoot residues vs. crop species for soil arthropod diversity and abundance of arable systems & Scheunemann N. et al. & 2015 & Soil Biology and Biochemistry & 81 & & $81-88$ & 10.1016/j.soilbio.2014.11.006 \\
\hline 439 & $\begin{array}{l}\text { Carbon dioxide exchange above a Mediterranean } \mathrm{C} 3 / \mathrm{C} 4 \text { grassland during two climatologically contrasting } \\
\text { years }\end{array}$ & Aires L. M. I. et al. & 2008 & Global Change Biology & 14 & 3 & $539-555$ & 10.1111/j.1365-2486.2007.01507.x \\
\hline 440 & Grassland Carbon Sequestration Ability in China: A New Perspective from Terrestrial Aridity Zones & Chen Y. et al. & 2016 & Rangeland Ecology and Management & 69 & 1 & $84-94$ & 10.1016/j.rama.2015.09.003 \\
\hline 441 & $\begin{array}{l}\text { Annual gross primary production and absorbition of solar energy by artemisia sp. in arid and semiarid } \\
\text { shrublands }\end{array}$ & Sanjerehei M. M. & 2013 & Applied Ecology and Environmental Research & 11 & 3 & $355-370$ & 10.15666/aeer/1103_355370 \\
\hline 442 & $\begin{array}{l}\text { Sensitivity of Temperate Desert Steppe Carbon Exchange to Seasonal Droughts and Precipitation Variations in } \\
\text { Inner Mongolia, China }\end{array}$ & Yang F. and Zhou G. & 2013 & PLOS One & 8 & 2 & e55418 & 10.1371/journal.pone. 0055418 \\
\hline 443 & $\begin{array}{l}\text { Primary production of Inner Mongolia, China, between } 1982 \text { and } 1999 \text { estimated by a satellite data-driven } \\
\text { light use efficiency model }\end{array}$ & Brogaard S. et al. & 2005 & Global and Planetary Change & 45 & 4 & $313-332$ & 10.1016/j.gloplacha.2004.09.012 \\
\hline 444 & $\begin{array}{l}\text { Net ecosystem } \mathrm{CO}_{2} \text { exchange in Mojave Desert shrublands during the eighth year of exposure to elevated } \\
\mathrm{CO} 2\end{array}$ & Jasoni R. L. et al. & 2005 & Global Change Biology & 11 & 5 & 749-756 & 10.1111/j.1365-2486.2005.00948.x \\
\hline 445 & $\begin{array}{l}\text { Precipitation Pattern Determines the Inter-annual Variation of Herbaceous Layer and Carbon Fluxes in a } \\
\text { Phreatophyte-Dominated Desert Ecosystem }\end{array}$ & Liu R. et al. & 2016 & Ecosystems & 19 & 4 & 601-614 & $10.1007 /$ s10021-015-9954-x \\
\hline 446 & $\begin{array}{l}\text { Irregular precipitation events in control of seasonal variations in } \mathrm{CO} 2 \text { exchange in a cold desert-shrub } \\
\text { ecosystem in northwest China }\end{array}$ & Xie J. et al. & 2015 & Journal of Arid Environments & 120 & & $33-41$ & 10.1016/j.jaridenv.2015.04.009 \\
\hline 447 & Phenological transition dictates the seasonal dynamics of ecosystem carbon exchange in a desert steppe & Huang G. and LiY. & 2015 & Journal of Vegetation Science & 26 & 2 & $337-347$ & $10.1111 /$ jvs.12236 \\
\hline 448 & Soil resource heterogeneity in the Mojave Desert & Titus. H. et al. & 2002 & Journal of Arid Environments & 52 & 3 & $269-292$ & 10.1006/jare.2002.1010 \\
\hline 449 & $\begin{array}{l}\text { Contrasting diel hysteresis between soil autotrophic and heterotrophic respiration in a desert ecosystem } \\
\text { under different rainfall scenarios }\end{array}$ & Song W. et al. & 2015 & Scientific Repots & 5 & & 16779 & $10.1038 /$ srep 16779 \\
\hline 450 & $\begin{array}{l}\text { Winter soil CO2 efflux and its contribution to annual soil respiration in different ecosystems of a forest-steppe } \\
\text { ecotone, north China }\end{array}$ & Qin L. et al. & 2015 & Eurasian Soil Science & 48 & 8 & $871-880$ & $10.1134 / \mathrm{S} 1064229315080050$ \\
\hline 451 & $\begin{array}{l}\text { Leaf litter decomposition in a southern Sonoran Desert ecosystem, northwestern Mexico: Effects of habitat } \\
\text { and litter quality }\end{array}$ & Martínez-Yrizar A. et al. & 2007 & Acta Oecologica & 32 & 3 & 291-300 & 10.1016/j.actao.2007.05.010 \\
\hline 452 & The contribution of trees and grasses to productivity of an Australian tropical savanna & Moore C. E. et al. & 2016 & Biogeosciences & 13 & 8 & $2387-2403$ & 10.5194/bg-13-2387-2016 \\
\hline 453 & Legume proportion, poultry litter, and tillage effects on cover crop decomposition & Poffenbarger H. J. et al. & 2015 & Agronomy Journal & 107 & 6 & 2083-2096 & 10.2134/agronj15.0065 \\
\hline 454 & $\begin{array}{l}\text { Organic farming fosters agroecosystem functioning in Argentinian temperate soils: Evidence from litter } \\
\text { decomposition and soil fauna }\end{array}$ & Domínguez A. et al. & 2014 & Applied Soil Ecology & 83 & & $170-176$ & 10.1016/j.apsoil.2013.11.008 \\
\hline 455 & Functional measures and food webs of high elevation springs in the Swiss alps & Robinson C. T. et al. & 2008 & Aquatic Sciences & 70 & 4 & $432-445$ & $10.1007 / 500027-008-8125-y$ \\
\hline 456 & $\begin{array}{l}\text { Discharge, legacy effects and nutrient availability as determinants of temporal patterns in biofilm metabolism } \\
\text { and accrual in an arctic river }\end{array}$ & Kendrick M. R. and Huryn A. D. & 2015 & Freshwater Biology & 60 & 11 & 2323-2336 & 10.1111/fwb.12659 \\
\hline 457 & $\begin{array}{l}\text { Nutrient and organic matter patterns across the Mackenzie River, estuary and shelf during the seasonal } \\
\text { recession of sea-ice }\end{array}$ & Emmerton C. A. et al. & 2008 & Journal of Marine Systems & 74 & & 741-755 & 10.1016/j.jmarsys.2007.10.001 \\
\hline
\end{tabular}




\begin{tabular}{|c|c|c|c|c|c|c|c|c|}
\hline 458 & Biological responses to fertilization of Oksrukuyik Creek, a tundra stream & Harvey C. J. et al. & 1998 & J. of the North Am. Benth. Soc. & 17 & 2 & $190-209$ & $10.2307 / 1467962$ \\
\hline 459 & Bacterial production and microbial food web structure in a large arctic river and the coastal Arctic Ocean & Vallières C. et al. & 2008 & Journal of Marine Systems & 74 & $3-4$ & $756-773$ & 10.1016/j.jmarsys.2007.12.002 \\
\hline 460 & $\begin{array}{l}\text { Heterotrophic bacterial activity and fluxes of dissolved free amino acids and glucose in the Arctic rivers Ob, } \\
\text { Yenisei and the adjacent Kara Sea }\end{array}$ & Meon B. and Amon R. M. W. & 2004 & Aquatic Microbial Ecology & 37 & 2 & $121-135$ & 10.3354/ame037121 \\
\hline 461 & Benthic and pelagic food resources for zooplankton in shallow high-latitude lakes and ponds & Rautio M. and Vincent W. F. & 2006 & Freshwater Biology & 51 & 6 & 1038-1052 & 10.1111/j.1365-2427.2006.01550.x \\
\hline 462 & Contribution of sediment respiration to summer $\mathrm{CO} 2 \mathrm{emission}$ from low productive boreal and subarctic lakes & s Algesten G. et al. & 2005 & Microbial Ecology & 50 & 4 & $529-535$ & $10.1007 / 500248-005-5007-x$ \\
\hline 463 & Dynamics of dissolved organic carbon lability in a eutrophic lake & Søndergaard M. et al. & 1995 & Limnology and Oceanography & 40 & 1 & $46-54$ & 10.4319/lo.1995.40.1.0046 \\
\hline 464 & Longitudinal patterns of organic matter transport and turnover along a New Zealand grassland river & Young R. G. and Huryn A. D. & 1997 & Freshwater Biology & 38 & & 93-107 & 10.1046/j.1365-2427.1997.00196.x \\
\hline 465 & $\begin{array}{l}\text { Impact of Wildfire on Stream Nutrient Chemistry and Ecosystem Metabolism in Boreal Forest Catchments of } \\
\text { Interior Alaska }\end{array}$ & Betts E. F. and Jones Jr J. B. & 2009 & Arctic, Antarctic, and Alpine Research & 41 & 4 & 407-417 & 10.1657/1938-4246-41.4.407 \\
\hline 466 & Recovery of macroinvertebrate communities from stream habitat restoration & Laasonen P. et al. & 1998 & $\begin{array}{l}\text { Aquatic Conservation: Marine and } \\
\text { Freshwater Ecosystems }\end{array}$ & 8 & 1 & $101-113$ & $\begin{array}{l}\text { 10.1002/(sici)1099- } \\
\text { 0755(199801/02)8:1<101::aid- } \\
\text { aqc251>3.0.co_2-4 }\end{array}$ \\
\hline 467 & Epilithic chlorophyll a, photosynthesis, and respiration in control and fertilized reaches of a tundra stream & Bowden W. B. et al. & 1992 & Hydrobiologia & 240 & & $121-131$ & 10.1007/BF00013457 \\
\hline 468 & The Effect of Invertebrate Predators on Leaf Litter Processing in an Alpine Stream & Obernborfer R. Y. et al. & 1984 & Ecology & 65 & 4 & $1325-1331$ & $10.2307 / 1938337$ \\
\hline 469 & $\begin{array}{l}\text { Litter quality, stream characteristics and litter diversity influence decomposition rates and } \\
\text { macroinvertebrates }\end{array}$ & Leroy C. J. and Marks J. C. & 2006 & Freshwater Biology & 51 & 4 & $605-617$ & 10.1111/j.1365-2427.2006.01512.x \\
\hline 470 & A cross-system comparison of bacterial and fungal biomass in detritus pools of headwater streams & Findlay S. et al. & 2002 & Microbial Ecology & 43 & & $55-66$ & 10.1007/10.007/s00248-001-1020-x \\
\hline 471 & Bryophytes and associated invertebrates in first-order alpine streams of Arthur 's Pass, New Zealand & Suren A. & 1993 & $\begin{array}{l}\text { New Zealand Journal of Marine and } \\
\text { Freshwater Research }\end{array}$ & 27 & 4 & 479-494 & 10.1080/00288330.1993.9516589 \\
\hline 472 & Black fly (Diptera: Simuliidae) response to phosphorus enrichment of an arctic tundra stream & Hiltner A. L. and Hershey A. E. & 1992 & Hydrobiologia & 240 & & $259-265$ & $10.1007 / \mathrm{BF} 00013467$ \\
\hline 473 & Production of fish stocks in Thingvallavatn, Iceland & Snorrason S. S. et al. & 1992 & Oikos & 64 & $1-2$ & $371-380$ & $10.2307 / 3545059$ \\
\hline 474 & $\begin{array}{l}\text { Quantifying effective restoration: reassessing the productive capacity of a constructed stream } 14 \text { years after } \\
\text { construction }\end{array}$ & Scrimgeour G. J. et al. & 2014 & Can. J. of Fish. and Aq. Sci. & 71 & 4 & 589-601 & 10.1139/cjfas-2013-0354 \\
\hline 475 & $\begin{array}{l}\text { Effect of tributary and braided con fl uences on aquatic macroinvertebrate communities and geomorphology } \\
\text { in an alpine river watershed }\end{array}$ & Clay P. A. et al. & 2015 & Freshwater Science & 34 & 3 & $845-856$ & 10.1086/682329. \\
\hline 476 & $\begin{array}{l}\text { Secondary production of highly unsaturated fatty acids by zoobenthos across rivers contrasting in } \\
\text { temperature }\end{array}$ & Gladyshev M. I. et al. & 2015 & River Research and Applications & 22 & & 1085-1095 & 10.1002/rra.2945 \\
\hline 477 & The macrobenthos of the pristine stream, Skiftessaa, Høylandet, Norway & Aagaard K. et al. & 1997 & Hydrobiologia & 348 & & $81-94$ & 10.1023/A:1003037200703 \\
\hline 478 & Mosses in High-Arctic lakes: in situ measurements of annual primary production and decomposition & Riis T. et al. & 2016 & Polar Biology & 39 & 3 & $543-552$ & $10.1007 / 500300-015-1806-9$ \\
\hline 479 & Slow growth and decomposition of mosses in Arctic lakes & Sand-Jensen K. et al. & 1999 & Can. J. of Fish. and Aq. Sci. & 56 & 3 & $388-393$ & $10.1139 / \mathrm{f} 98-184$ \\
\hline 480 & The processes and control of the microbial colonization and decomposition of plant litter in an arctic lake & Federle T. W. and Vestal J. R. & 1980 & Applied and Environmental Microbiology & 39 & 4 & $888-893$ & \\
\hline 481 & Temperature sensitivity of organic carbon mineralization in contrasting lake sediments & Gudasz C. et al. & 2015 & $\begin{array}{l}\text { Journal of Geophysical Research: } \\
\text { Biogeosciences }\end{array}$ & 120 & & $1215-1225$ & $10.1002 / 2015 J G 002928$ \\
\hline 482 & Benthic secondary production in eight oligotrophic arctic Alaskan lakes & Northington R. M. et al. & 2010 & J. of the North Am. Benth. Soc. & 29 & 2 & $465-479$ & 10.1899/09-026.1 \\
\hline 483 & $\begin{array}{l}\text { Ecological studies on the decomposition rate of fish carcasses by benthic organisms in the littoral zone of } \\
\text { Lake Constance, Germany }\end{array}$ & Premke K. et al. & 2010 & J. of the North Am. Benth. Soc. & 46 & 3 & $157-168$ & 10.1051/limn/2010017 \\
\hline 484 & Fish decomposition in boreal lakes and biogeochemical implications & Chidami S. and Amyot M. & 2008 & Limnology and Oceanography & 53 & 5 & 1988-1996 & 10.4319/lo.2008.53.5.1988 \\
\hline 485 & $\begin{array}{l}\text { Whole-lake mineralization of allochthonous and autochthonous organic carbon in a large humic lake } \\
\text { (Örträsket, N. Sweden) }\end{array}$ & Jonsson A. et al. & 2001 & Limnology and Oceanography & 46 & 7 & $1691-1700$ & 10.4319/lo.2001.46.7.1691 \\
\hline 486 & $\begin{array}{l}\text { Organic matter characterization and decomposition dynamics in sub Antarctic streams impacted by invasive } \\
\text { beavers }\end{array}$ & Ulloa E. et al. & 2012 & Latin American Journal of Aquatic Research & 40 & 4 & 881-892 & 10.3856/vol40-issue4-fulltext-6 \\
\hline 487 & Can pelagic net heterotrophy account for carbon fluxes from eastern Canadian lakes? & Dubois K. et al. & 2009 & Applied Geochemistry & 24 & 5 & $988-998$ & 10.1016/j.apgeochem.2009.03.001 \\
\hline 488 & $\begin{array}{l}\text { Ecosystem metabolism and greenhouse gas production in a mesotrophic northern temperate lake } \\
\text { experiencing seasonal hypoxia }\end{array}$ & Salk K. R. et al. & 2016 & Biogeochemistry & 131 & 3 & 303-319 & 10.1007/s10533-016-0280-y \\
\hline 489 & $\begin{array}{l}\text { Plankton metabolism and physical forcing in a productive embayment of a large oligotrophic lake: insights } \\
\text { from stable oxygen isotopes }\end{array}$ & Bocaniov S. A. et al. & 2012 & Freshwater Biology & 57 & 3 & 481-496 & 10.1111/j.1365-2427.2011.02715.x \\
\hline 490 & $\begin{array}{l}\text { Consequences for pelagic energy mobilisation of a sudden browning episode without a clear increase in DOC } \\
\text { concentration: a case of a boreal pristine lake }\end{array}$ & Peltomaa E. and Ojala A. & 2016 & Aquatic Sciences & 78 & 4 & 627-639 & 10.1007/s00027-015-0452-1 \\
\hline 491 & The Stoichiometry of Carbon, Nitrogen, and Phosphorus in Particulate Matter of Lakes and Oceans & Hecky R. E. et al. & 1993 & Limnology and Oceanography & 38 & 4 & $709-724$ & 10.4319/lo.1993.38.4.0709 \\
\hline 492 & Organic carbon decomposition rates controlled by water retention time across inland waters & Catalán N. et al. & 2016 & Nature Geoscience & 9 & May & $1-7$ & $10.1038 /$ ngeo2720 \\
\hline
\end{tabular}




\begin{tabular}{|c|c|c|c|c|c|c|c|c|}
\hline 493 & Effects of Acid on Plant Litter Decomposition in an Arctic Lake & Mckinley V. L. and Vaestal J. R. & 1982 & Applied and Environmental Microbiology & 43 & 5 & 1188-1195 & \\
\hline 494 & $\begin{array}{l}\text { Biogenic carbon flows through the planktonic food web of the Amundsen Gulf (Arctic Ocean): A synthesis of } \\
\text { field measurements and inverse modeling analyses }\end{array}$ & Forest A. et al. & 2011 & Progress in Oceanography & 91 & 4 & $410-436$ & 10.1016/j.pocean.2011.05.002 \\
\hline 495 & Salp contributions to vertical carbon flux in the Sargasso Sea & Stone J. P. and Steinberg D. K. & 2016 & $\begin{array}{l}\text { Deep-Sea Research Part I: Oceanographic } \\
\text { Research Papers }\end{array}$ & 113 & & $90-100$ & 10.1016/j.dsr.2016.04.007 \\
\hline 496 & Decomposition rate of the giant jellyfish Nemopilema nomurai in Sado Island. & Igushi N. et al. & 2006 & $\begin{array}{l}\text { FRA, Japan Sea National Fisheries Research } \\
\text { Institute report. }\end{array}$ & & & & $\begin{array}{l}\text { http://jsnfri.fra.affrc.go.jp/Kurage/ } \\
\text { kurage_hp18/Sado_bunkai.pdf. }\end{array}$ \\
\hline 497 & Response of Bacterial Communities to Different Detritus Compositions in Arctic Deep-Sea Sediments & Hoffmann K. et al. & 2017 & Frontiers in Microbiology & 8 & $\begin{array}{l}\text { Febru } \\
\text { ary }\end{array}$ & Art.266 & 10.3389/fmicb.2017.00266 \\
\hline 498 & 'Total' sediment biomass and preliminary estimates of organic carbon residence time in deep-sea benthos & Rowe G. et al. & 1991 & Marine Ecology Progress Series & 79 & $1-2$ & 99-114 & 10.3354/meps079099 \\
\hline 499 & Fish food in the deep sea: Revisiting the role of large food-falls & Higgs N. D. et al. & 2014 & PlosOne & 9 & 5 & e96016 & 10.1371/journal.pone.0096016 \\
\hline 500 & Food for the deep sea: utilisation, dispersal and flux of necton falls at the Santa Catalina Basin floor & Smith C. R. & 1985 & Deep-Sea Research & 32 & 4 & $417-442$ & 10.1371/journal.pone.0096016 \\
\hline 501 & Trophic flows, kelp culture and fisheries in the marine ecosystem of an artificial reef zone in the Yellow Sea & Wu Z. et al. & 2016 & Estuarine, Coastal and Shelf Science & 182 & & $86-97$ & 10.1016/j.ecss.2016.08.021 \\
\hline 502 & $\begin{array}{l}\text { Macroscopic network properties and short-term dynamic simulations in coastal ecological systems at Fildes } \\
\text { Bay (King George Island, Antarctica) }\end{array}$ & Ortiz M. et al. & 2016 & Ecological Complexity & 28 & & $145-157$ & 10.1016/j.ecocom.2016.06.003 \\
\hline 503 & Trophic network model of the Northern Adriatic Sea: Analysis of an exploited and eutrophic ecosystem & Barausse A. et al. & 2009 & Estuarine, Coastal and Shelf Science & 83 & 4 & $577-590$ & 10.1016/j.ecss.2009.05.003 \\
\hline 504 & Food-web dynamics in the South Catalan Sea ecosystem (NW Mediterranean) for 1978-2003 & Coll M. et al. & 2008 & Ecological Complexity & 217 & $1-2$ & $95-116$ & 10.1016/j.ecolmodel.2008.06.013 \\
\hline 505 & Anthropogenic Enrichment Alters a Marine Benthic Food Web & Tewfik A. et al. & 2013 & Ecology & 86 & 10 & $2726-2736$ & 10.1890/04-1537 \\
\hline 506 & $\begin{array}{l}\text { Meiofaunal production and energy transfer efficiency in a seagrass Posidonia oceanica bed in the western } \\
\text { Mediterranean }\end{array}$ & Danovaro R. et al. & 2002 & Marine Ecology Progress Series & 234 & & 95-104 & 10.3354/meps234095 \\
\hline 507 & Ecosystem trophic structure and energy flux in the Northern Gulf of California, Mexico & Morales-Zárate M. V. et al. & 2004 & Ecological Modelling & 174 & 4 & $331-345$ & 10.1016/j.ecolmodel.2003.09.028 \\
\hline 508 & A preliminary trophic model of the continental shelf, south- western Gulf of Mexico & Manickchand-Heileman S. et al. & 1998 & Estuarine, Coastal and Shelf Science & 46 & 6 & 885-899 & 10.1006/ecss.1997.0324 \\
\hline 509 & $\begin{array}{l}\text { Balance between autotrophic and heterotrophic components and processes in microbenthic communities of } \\
\text { sandy sediments: a field study. }\end{array}$ & Sundbäck K. et al. & 1996 & Estuarine, Coastal and Shelf Science & 43 & & $689-706$ & 10.1006/ecss.1996.0097 \\
\hline 510 & Macrobenthic secondary production in the intertidal zone of the Ria Formosa - a lagoon in southern Portugal & Sprung $\mathrm{M}$. & 1994 & Estuarine, Coastal and Shelf Science & 38 & & $539-558$ & 10.1006/ecss.1994.1037 \\
\hline 511 & $\begin{array}{l}\text { Estudio ecológico ele la macrofauna del estuario de La Foz (NO de España):L Composición, estructura, } \\
\text { variación estacional y producción de las comunidades }\end{array}$ & Anadón $\mathrm{R}$. & 1986 & Investigacion Pesquera & 44 & 3 & 407-444 & \\
\hline 512 & $\begin{array}{l}\text { The Influence of Allochthonous Leaf Detritus on the Occurrence of Crustacean Detritivores in the Soft-bottom } \\
\text { Macrobenthos of the Po River Delta Area (northwestern Adriatic Sea) }\end{array}$ & Mancinelli G. and Rossi L. & 2002 & Estuarine, Coastal and Shelf Science & 54 & 5 & 849-861 & 10.1006/ecss.2001.0861 \\
\hline 513 & Tidal influence on particulate organic carbon export fluxes around a tall seamount & Turnewitsch R. et al. & 2016 & Progress in Oceanography & 149 & & $189-213$ & 10.1016/j.pocean.2016.10.009 \\
\hline 514 & $\begin{array}{l}\text { Vertical Carbon Flux of Biogenic Matter in a Coastal Area of the Aegean Sea: The Importance of } \\
\text { Appendicularians }\end{array}$ & Zervoudaki S. et al. & 2014 & Estuaries and Coasts & 37 & 4 & 911-924 & 10.1007/s12237-013-9723-z \\
\hline 515 & Microbial respiration and trophic regimes in the Northern Adriatic Sea (Mediterranean Sea) & La Ferla R. et al. & 2006 & Estuarine, Coastal and Shelf Science & 69 & $1-2$ & $196-204$ & 10.1016/j.ecss.2006.04.005 \\
\hline 516 & $\begin{array}{l}\text { Microbial contribution to carbon biogeochemistry in the Central Mediterranean Sea: Variability of activities } \\
\text { and biomass }\end{array}$ & La Ferla R. et al. & 2005 & Journal of Marine Research & 57 & $1-2$ & $146-166$ & 10.1016/j.jmarsys.2005.05.001 \\
\hline 517 & $\begin{array}{l}\text { Emergence of amphibiotic insects from a floodplain lake in the Usman Forest in the Central Russian forest } \\
\text { steppe }\end{array}$ & Silina A. E. & 2016 & Contemporary Problems of Ecology & 9 & 4 & 421-436 & 10.1134/\$1995425516040107 \\
\hline 518 & $\begin{array}{l}\text { Emergence of Trichoptera from Findley Lake and two ponds in the coniferous forest of the Cascade } \\
\text { Mountains, USA }\end{array}$ & Sherk T. and Rau G. & 1991 & $\begin{array}{l}\text { Proceedings of the 6th International } \\
\text { Symposium on Trichoptera }\end{array}$ & 26 & $2-4$ & $321-330$ & \\
\hline 519 & Macrobenthos. In: Hobbie et al. Limnology of tundra ponds: Barrow, Alaska & Butler M. et al. & 1980 & $\begin{array}{l}\text { In: Hobbie et al. Limnology of tundra ponds: } \\
\text { Barrow, Alaska }\end{array}$ & $\begin{array}{c}\text { chapter } \\
7\end{array}$ & & 251-298 & \\
\hline 520 & $\begin{array}{l}\text { Boomerang ecosystem fluxes: Organic carbon inputs from land to lakes are returned to terrestrial food webs } \\
\text { via aquatic insects }\end{array}$ & Scharnweber K. et al. & 2014 & Oikos & 123 & & 1439-1448 & 10.1111/oik.01524 \\
\hline 521 & Dispersal of Terrestrial Plant Litter into a Subalpine Lake & Rau G. H. & 1976 & Oikos & 27 & 1 & $153-160$ & $10.2307 / 3543445$ \\
\hline 522 & Airborne carbon deposition on a remote forested lake & Preston N. D. et al. & 2008 & Aquatic sciences & 70 & 3 & $213-224$ & $10.1007 / 500027-008-8074-5$ \\
\hline 523 & Mangrove (Avicennia marina subsp. australasica) litter production and decomposition in a temperate estuary & Gladstone-Gallagher R. V. et al. & 2013 & $\begin{array}{l}\text { New Zealand Journal of Marine and } \\
\text { Freshwater Research }\end{array}$ & 48 & 1 & 24-37 & 10.1080/00288330.2013.827124 \\
\hline 524 & $\begin{array}{l}\text { Substance and energy flows formed by the emergence of amphibiotic insects across the water-air boundary } \\
\text { on the floodplain lakes of the Volga River }\end{array}$ & Djomina I. V. et al. & 2016 & Contemporary Problems of Ecology & 9 & 4 & $407-420$ & 10.1134/\$1995425516040053 \\
\hline 525 & Periodical cicada detritus impacts stream ecosystem metabolism & Menninger H. L. et al. & 2008 & Ecosystems & 11 & & $1306-1317$ & 10.1007/s10021-008-9194-4 \\
\hline
\end{tabular}




\begin{tabular}{|c|c|c|c|c|c|c|c|c|}
\hline 526 & $\begin{array}{l}\text { Invasive European bird cherry (Prunus padus) reduces terrestrial prey subsidies to urban Alaskan salmon } \\
\text { streams }\end{array}$ & Roon D. A. et al. & 2016 & Can. J. of Fish. and Aq. Sci. & 73 & 11 & $1679-1690$ & 10.1139/cjfas-2015-0548 \\
\hline 527 & $\begin{array}{l}\text { A terrestrial-aquatic food web subsidy is potentially mediated by multiple predator effects on an arboreal } \\
\text { crab }\end{array}$ & Yeager L. A. et al. & 2016 & $\begin{array}{l}\text { Journal of Experimental Marine Biology and } \\
\text { Ecology }\end{array}$ & 475 & & 73-79 & 10.1016/j.jembe.2015.10.017 \\
\hline 528 & How hydroperiod and species richness affect the balance of resource flows across aquatic-terrestrial habitats & Schriever T. A. et al. & 2014 & Aquatic sciences & 76 & 1 & $131-143$ & 10.1007/s00027-013-0320-9 \\
\hline 529 & Above and belowground organic matter and root, shoot ratio in a cerrado in Central Brazil. & Abdala G. C. et al. & 1998 & Brazilian Journal of Ecology & 2 & 1 & & $\begin{array}{l}\text { http://www.seb- } \\
\text { ecologia.org.br/revista2/art2.html }\end{array}$ \\
\hline 530 & Biomass, nutrient pools and response to fire in the Brazilian cerrado & Castro de E. A. & 1996 & MSc Thesis, Oregon State University, USA & & & & http://hdl.handle.net/1957/35021 \\
\hline 531 & An African savannas: synthesis of the Nylsvley study. & Scholes R. J. and Walker B. H. & 1993 & $\begin{array}{l}\text { book, Cambridge University press, } \\
\text { Cambridge, UK }\end{array}$ & & & & 10.1017/CBO9780511565472 \\
\hline 532 & $\begin{array}{l}\text { Reconstructing the seasonal dynamics and relative contribution of the major processes sustaining } \mathrm{CO2} \\
\text { emissions in northern lakes }\end{array}$ & Vachon D. et al. & 2016 & Limnology and Oceanography & 62 & 2 & $706-722$ & 10.1002/Ino.10454 \\
\hline 533 & The role of sediments in the carbon budget of a small boreal lake & Chmiel H. E. et al. & 2016 & Limnology and Oceanography & 61 & 5 & $1814-1825$ & 10.1002/Ino.10336 \\
\hline 534 & $\begin{array}{l}\text { Seasonality of photochemical dissolved organic carbon mineralization and its relative contribution to pelagic } \\
\mathrm{CO} 2 \text { production in northern lakes }\end{array}$ & Vachon D. et al. & 2016 & $\begin{array}{l}\text { Journal of Geophysical Research: } \\
\text { Biogeosciences }\end{array}$ & 121 & 3 & $864-878$ & 10.1002/2015JG003244 \\
\hline 535 & High net $\mathrm{CO} 2$ and $\mathrm{CH} 4$ release at a eutrophic shallow lake on a formerly drained fen & Franz D. et al. & 2016 & Biogeosciences & 13 & 10 & 3051-3070 & 10.5194/bg-13-3051-2016 \\
\hline 536 & $\begin{array}{l}\text { Metabolic and physiochemical responses to a whole-lake experimental increase in dissolved organic carbon in } \\
\text { a north-temperate lake }\end{array}$ & Zwart J. A. et al. & 2016 & Limnology and Oceanography & 61 & 2 & 723-734 & 10.1002/Ino.10248 \\
\hline 537 & $\begin{array}{l}\text { Assessment of Top-Down and Bottom-Up Controls on the Collapse of Alewives (Alosa pseudoharengus) in } \\
\text { Lake Huron }\end{array}$ & Kao Y.-C. et al. & 2016 & Ecosystems & 19 & 5 & 803-831 & 10.1007/s10021-016-9969-y \\
\hline 538 & $\begin{array}{l}\text { Application of Ecopath model on trophic interactions and energy flows of impounded Manwan reservoir } \\
\text { ecosystem in Lancang River, southwest China }\end{array}$ & Deng L. et al. & 2015 & Journal of Freshwater Ecology & 30 & 2 & 281-297 & 10.1080/02705060.2014.942893 \\
\hline 539 & Contributions of mesozooplankton to vertical carbon export in a coastal upwelling system & Stukel M. R. et al. & 2013 & Marine Ecology Progress Series & 491 & & $47-65$ & $10.3354 /$ meps10453 \\
\hline 540 & Responses of a beaded Arctic stream to short-term $N$ and $P$ fertilisation & Benstead J. P. et al. & 2005 & Freshwater Biology & 50 & 2 & $277-290$ & $10.1111 / \mathrm{j} .1365-2427.2004 .01319 . x$ \\
\hline 541 & $\begin{array}{l}\text { Organic carbon flux and particulate organic matter composition in Arctic valley glaciers: Examples from the } \\
\text { Bayelva River and adjacent Kongsfjorden }\end{array}$ & Zhu Z.-Y. et al. & 2016 & Biogeosciences & 13 & 4 & 975-987 & $10.5194 / \mathrm{bg}-13-975-2016$ \\
\hline 542 & Seasonal variation of allochthonous and autochthonous energy inputs in an Alpine stream & Fenoglio S. et al. & 2015 & Journal of Limnology & 74 & 2 & $272-277$ & 10.4081/jlimnol.2014.1082 \\
\hline 543 & $\begin{array}{l}\text { Seasonal fluxes and age of particulate organic carbon exported from Arctic catchments impacted by localized } \\
\text { permafrost slope disturbances }\end{array}$ & $\begin{array}{l}\text { Lamoureux S. F. and Lafrenière } \\
\text { M. J. }\end{array}$ & 2014 & Environmental Research Letters & 9 & 4 & 45002 & 10.1088/1748-9326/9/4/045002 \\
\hline 544 & $\begin{array}{l}\text { Recent particulate organic carbon and total suspended matter fluxes from the Ob and Yenisei Rivers into the } \\
\text { Kara Sea (Siberia) }\end{array}$ & Gebhart A. C. et al. & 2004 & Marine Geology & 207 & & $225-245$ & 10.1016/j.margeo.2004.03.010 \\
\hline 545 & $\begin{array}{l}\text { Precipitation and net ecosystem exchange are the most important drivers of DOC flux in upland boreal } \\
\text { catchments }\end{array}$ & Pumpanen J. et al. & 2014 & $\begin{array}{l}\text { Journal of Geophysical Research: } \\
\text { Biogeosciences }\end{array}$ & 119 & & 1861-1878 & 10.1002/2014JG002705 \\
\hline 546 & Sunlight controls water column processing of carbon in arctic fresh waters & Cory R. M. et al. & 2014 & Science & 345 & 6199 & $925-928$ & $10.1126 /$ science. 1253119 \\
\hline 547 & $\begin{array}{l}\text { Environmental controls of whole-stream metabolism identified from continuous monitoring of Basque } \\
\text { streams Oihana }\end{array}$ & Izagirre O. et al. & 2008 & J. of the North Am. Benth. Soc. & 27 & 2 & $252-268$ & 10.1899/07-022.1 \\
\hline 548 & Temperature and the metabolic balance of streams & Demars B. O. L. et al. & 2011 & Freshwater Biology & 56 & 6 & $1106-1121$ & 10.1111/j.1365-2427.2010.02554.x \\
\hline 549 & Ecosystem Alteration of Boreal Forest Streams by Beaver ( Castor Canadensis ) & Naiman R. J. et al & 1986 & Ecology & 67 & 5 & 1254-1269 & $10.2307 / 1938681$ \\
\hline 550 & $\begin{array}{l}\text { Bacterial production and losses to predators along an open ocean productivity gradient in the Subtropical } \\
\text { North East Atlantic Ocean }\end{array}$ & Vaqué $\mathrm{D}$. et al. & 2014 & Journal of Plankton Research & 36 & 1 & $198-213$ & 10.1093/plankt/fbt085 \\
\hline 551 & High bacterial production, uptake and concentrations of dissolved organic matter in the Central Arctic Ocean & Rich J. et al. & 1997 & $\begin{array}{l}\text { Deep-Sea Research Part II: Topical Studies in } \\
\text { Oceanography }\end{array}$ & 44 & 8 & $1645-1663$ & 10.1016/S0967-0645(97)00058-1 \\
\hline 552 & Plankton respiration and carbon flux through bacterioplankton on the Louisiana shelf & Biddanda B. et al. & 1994 & Limnology and Oceanography & 39 & 6 & $1259-1275$ & 10.4319/lo.1994.39.6.1259 \\
\hline 553 & $\begin{array}{l}\text { Nutrient pulses driven by internal solitary waves enhance heterotrophic bacterial growth in the South China } \\
\text { Sea }\end{array}$ & Chen T.-Y. et al. & 2016 & Environmental Microbiology & 18 & 12 & 4312-4323 & 10.1111/1462-2920.13273 \\
\hline 554 & $\begin{array}{l}\text { Heterotrophic bacterial responses to the winter-spring phytoplankton bloom in open waters of the NW } \\
\text { Mediterranean }\end{array}$ & Gomes A. et al. & 2015 & $\begin{array}{l}\text { Deep-Sea Research Part l: Oceanographic } \\
\text { Research Papers }\end{array}$ & 95 & & $59-68$ & 10.1016/j.dsr.2014.11.007 \\
\hline 555 & $\begin{array}{l}\text { Heterotrophic bacterial production and metabolic balance during the VAHINE mesocosm experiment in the } \\
\text { New Caledonia lagoon }\end{array}$ & Van Wambeke F. et al. & 2016 & Biogeosciences & 13 & 11 & $3187-3202$ & $10.5194 / \mathrm{bg}-13-3187-2016$ \\
\hline 556 & $\begin{array}{l}\text { Annual mass drownings of the Serengeti wildebeest migration influence nutrient cycling and storage in the } \\
\text { Mara River }\end{array}$ & Subaluski A. L. et al. & 2017 & $\begin{array}{l}\text { Proceedings of the National Academy of } \\
\text { Science }\end{array}$ & 114 & 29 & 7647-7652 & 10.1073/pnas.1614778114 \\
\hline
\end{tabular}


